\title{
LINE ANTIDERIVATIONS OVER LOCAL FIELDS AND THEIR APPLICATIONS
}

\author{
S. V. LUDKOVSKY
}

Received 23 December 2003

A non-Archimedean antiderivational line analog of the Cauchy-type line integration is defined and investigated over local fields. Classes of non-Archimedean holomorphic functions are defined and studied. Residues of functions are studied; Laurent series representations are described. Moreover, non-Archimedean antiderivational analogs of integral representations of functions and differential forms such as the Cauchy-Green, MartinelliBochner, Leray, Koppelman, and Koppelman-Leray formulas are investigated. Applications to manifold and operator theories are studied.

\section{Introduction}

Line (Cauchy) integration is the cornerstone in the complex analysis and integral formulas of functions and differential forms such as the Cauchy-Green, Martinelli-Bochner, Leray, Koppelman, and Koppelman-Leray formulas play a very important role in it and in analysis of complex manifolds and the theory of Stein and Kähler manifolds and the theory of holomorphic functions (see, e.g., $[8,23,24]$ ). In the non-Archimedean case there is a not-so-developed analog of complex analysis. There are few works devoted to non-Archimedean holomorphic functions over the complex non-Archimedean field $\mathbb{C}_{\mathbf{p}}$ and the Levi-Civitá fields, which are not locally compact (see $[2,11]$ and the references therein). In those works M. M. Vishik and M. Berz have obtained analogs of residues and the Cauchy formula, but the integrals that they have used were of combinatorial-algebraic nature and they have operated with power series mainly for their analogs of holomorphic functions. On the other hand, there is no measure equivalent to the Haar measure on such nonlocally compact fields because of the Weil [29] theorem stating that the existence of such nontrivial measure on a topological group implies its local compactness. This paper is devoted to other non-Archimedean analogs of integral representation theorems that were not yet considered by other authors. Moreover, this paper operates with locally compact non-Archimedean fields of characteristic zero (local fields) and the corresponding analogs of complex planes. Apart from the classical case in the non-Archimedean case there is no indefinite integral. Antiderivation operators by Schikhof [22] are used instead. 
It is necessary to note that this paper considers not only manifolds treated by the rigid geometry but also much wider classes continuing the previous work [19]. The existence of an exponential mapping for these manifolds is proved. A rigid non-Archimedean geometry serves mainly the needs of the cohomology theory on such manifolds, but it is too restrictive and operates with narrow classes of analytic functions [7]. It was introduced at the beginning of the sixties of the 20th century. Few years later on wider classes of functions were investigated by Schikhof [22]. In this paper, classes of functions and antiderivation operators by Schikhof and their generalizations from $[14,15,16]$ are used.

Section 2 is devoted to the definition and investigations of the non-Archimedean analogs of the line integration over local fields. Classes of non-Archimedean holomorphic functions are defined and studied. For this specific non-Archimedean geometry definitions and theorems are given (see also definitions and notations in $[13,14,15,16,18,19]$ ). It is necessary to note that definitions, formulations of theorems, propositions, and so forth. and their proofs differ substantially from the classical case (over $\mathbb{C}$ ). Residues of functions are studied and Laurent series representations are described. In Section 3, nonArchimedean antiderivational analogs of integral representations of functions and differential forms such as the Cauchy-Green, Martinelli-Bochner, Leray, Koppelman, and Koppelman-Leray formulas are investigated. These studies are accomplished on domains in finite-dimensional Banach spaces over local fields and also on manifolds over local fields. All results of this paper are obtained for the first time. Finally applications of the obtained results to the theory of non-Archimedean manifolds and linear operators in non-Archimedean Banach spaces are outlined. In works of Vishik (see [11] and the references therein) the theory of non-Archimedean (Krasner) analytic operators with compact spectra in $\mathbb{C}_{\mathbf{p}}$ was developed. In this paper, operators may have noncompact spectra in a field $\mathbf{L}$ such that $\mathbb{Q}_{\mathbf{p}} \subset \mathbf{L}$ (maybe also $\mathbf{L} \supset \mathbb{C}_{\mathbf{p}}$ and $\mathbf{L} \neq \mathbb{C}_{\mathbf{p}}$ ) continuing the investigation of [12].

\section{Line antiderivation over local fields}

To avoid misunderstandings we first present our specific definitions.

2.1. Notation and remarks. Let $\mathrm{K}$ denote a local field, that is, a finite algebraic extension of the field $\mathbb{Q}_{\mathbf{p}}$ of $p$-adic numbers with a norm extending that of $\mathbb{Q}_{\mathbf{p}}$ [30]. Denote by $\mathbb{C}_{\mathbf{p}}$ the field of complex numbers with the norm extending that of $\mathbb{Q}_{\mathbf{p}}[10]$. If $i \in \mathbf{K}$, take $\alpha \in \mathbb{C}_{\mathbf{p}} \backslash \mathbf{K}$ such that there exists $\tilde{m} \in \mathbb{N}$ with $\alpha^{\tilde{m}} \in \mathbf{K}$, where $\tilde{m}$ is a minimal natural number, $\tilde{m}=\tilde{m}(\alpha), i:=(-1)^{1 / 2}$. If $i \notin \mathbf{K}$, take $\alpha=i$. Denote by $\mathbf{K}(\alpha)$ a local field which is the extension of $\mathbf{K}$ with the help of $\alpha$.

Suppose $U$ is a clopen compact perfect (i.e., dense in itself) subset in $\mathbf{K}$ and ${ }_{U} \sigma:=\sigma$ is its approximation of the identity: there is a sequence of maps $\sigma_{l}: U \rightarrow U$, where $0 \leq l \in \mathbb{Z}$, such that

(i) $\sigma_{0}$ is constant;

(ii) $\sigma_{l} \circ \sigma_{n}=\sigma_{n} \circ \sigma_{l}=\sigma_{n}$ for each $l \geq n$;

(iii) there exists a constant $0<\rho<1$ such that for each $x, y \in U$ the inequality $\mid x-$ $y \mid<\rho^{n}$ implies $\sigma_{n}(x)=\sigma_{n}(y)$; 
(iv) $\left|\sigma_{n}(x)-x\right|<\rho^{n}$ for each integer $n \geq 0$. Consider spaces $C^{n}(U, \mathbf{L})$ of all $n$-times continuously differentiable in the sense of difference quotients functions $f: U \rightarrow$ $\mathbf{L}$, where $\mathbf{L}$ is a field containing $\mathbf{K}$ with the multiplicative norm $|\cdot|_{\mathbf{L}}$ which is the extension of the multiplicative norm $|\cdot|_{\mathbf{K}}$ in $\mathbf{K}$. Then there exists an antiderivation

$$
{ }_{U} P^{n}: C^{n-1}(U, \mathbf{L}) \rightarrow C^{n}(U, \mathbf{L})
$$

given by the formula

$$
{ }_{U} P^{n} f(x):=\sum_{l=0}^{\infty} \sum_{j=0}^{n-1} \frac{f^{(j)}\left(x_{l}\right)\left(x_{l+1}-x_{l}\right)^{j+1}}{(j+1) !}
$$

where $x_{l}:=\sigma_{l}(x), x \in U, n \geq 1$ (see [22, Section 80]). Formula (2.2) shows that if ${ }_{U} P^{n}$ is defined on $C^{n-1}(U, \mathbf{K})$, then it is defined on $C^{n-1}(U, Y)$ for each field $\mathbf{L}$ which is complete relative to its norm such that $\mathbf{K} \subset \mathbf{L}$ and a Banach space $Y$ over $\mathbf{L}$. From $C^{m}(U, Y) \subset$ $C^{n-1}(U, Y)$ for each $\infty \geq m \geq n \in \mathbb{N}$, it follows that there exists a restriction $\left.{ }_{U} P^{n}\right|_{C^{m}}$ : $C^{m}(U, Y) \rightarrow C^{n}(U, Y)$ which is the L-linear operator for each $m \geq n-1$.

Since $P^{n}$ is the $\mathbf{L}$-linear continuous operator from $C^{n-1}$ into $C^{n}$, then there exists the L-linear space ${ }_{P} C_{0}^{n}(U, Y):=P^{n}\left(C^{n-1}(U, Y)\right)$, putting ${ }_{P} C^{n}(U, Y):={ }_{P} C_{0}^{n}(U, Y) \oplus Y$, where $n \geq 1, Y$ is a Banach space over $\mathbf{L}$. For a clopen subset $\Omega$ in $(\mathbf{K} \oplus \alpha \mathbf{K})^{m}$ such that $\Omega \subset$ $U^{m} \times U^{m}$ consider the antiderivation ${ }_{\Omega} P^{n} f(z)$ as the restriction of $U_{U^{m} \times U^{m}} P^{n} f(z)$ on $\Omega$,

$$
{ }_{\Omega} P^{n} f(z):=\left.{ }_{U^{m} \times U^{m}} P^{n}\right|_{\Omega} f(z)=U_{U^{m} \times U^{m}} P^{n} f(z) \chi_{\Omega}(z),
$$

where

$$
U^{m} \times U^{m} P^{n} f(z):={ }_{U} P_{x_{1}}^{n} \cdots{ }_{U} P_{x_{m}}^{n} P_{y_{1}}^{n} \cdots{ }_{U} P_{y_{m}}^{n} f(z),
$$

$\chi_{\Omega}(z)$ denotes the characteristic function of $\Omega, \chi_{\Omega}(z)=1$ for each $z \in \Omega, \chi_{\Omega}(z)=0$ for each $z \in \mathbf{K}^{2 m} \backslash \Omega, z=(x, y), x, y \in U^{m} \subset \mathbf{K}^{m}, x=\left(x_{1}, \ldots, x_{m}\right), x_{1}, \ldots, x_{m} \in \mathbf{K}$, and ${ }_{U} P_{x_{l}}^{n}$ means the antiderivation by the variable $x_{l}$. This is correct, since each $f \in C^{(0, n-1)}(\Omega, \mathbf{L})$ : $=C((0, n-1), \Omega \rightarrow \mathbf{L})$ (see [15, Section I.2.4] and [16]) has a $C^{(0, n-1)}$-extension on $U^{m} \times U^{m}$, for example, $\left.f\right|_{U^{m} \times U^{m} \backslash \Omega}=0$. This means that $U^{m} \times U^{m} P^{n} f(z)$ is the antiderivation defined with the help of approximation of the unity on $U^{m} \times U^{m}$ such that $U^{m} \times U^{m} \sigma=$ $\left({ }_{U} \sigma, \ldots, U \sigma\right)$.

The condition of compactness of $\Omega$ is not very restrictive, since each locally compact subset in $(\mathbf{K} \oplus \alpha \mathbf{K})^{m}$ has a one-point (Alexandroff) compactification which is totally disconnected and hence homeomorphic to a clopen subset in $(\mathbf{K} \oplus \alpha \mathbf{K})^{m}$ (see [5, Section 3.5 and Theorem 6.2.16] about universality of the Cantor cube). If $\rho\left(z_{1}, z_{2}\right):=\left|z_{1}-z_{2}\right|$ is the metric in $(\mathbf{K} \oplus \alpha \mathbf{K})^{m}$, then the metric $\rho^{\prime}\left(z_{1}, z_{2}\right):=\rho\left(z_{1}, z_{2}\right) /\left[1+\rho\left(z_{1}, z_{2}\right)\right]$ has the extension on the one-point compactification $A(\mathbf{K} \oplus \alpha \mathbf{K})^{m}:=(\mathbf{K} \oplus \alpha \mathbf{K})^{m} \cup\{A\}$, where $A$ is a singleton. If $Y$ is a metric space with a metric $\rho$, then $B(Y, y, r):=\{z \in Y: \rho(z, y) \leq r\}$ denotes the ball of radius $r>0$ and containing a point $y \in Y$. 


\subsection{Notes and definitions.}

2.2.1. For a local field $\mathbf{K}$ there exists a prime $p$ such that $\mathbf{K}$ is a finite algebraic extension of $\mathbb{Q}_{\mathbf{p}}$. In view of [30, Theorems 1.1 and 4.6 and Proposition 4.4] there exists a prime element $\pi \in \mathbf{K}$ such that $P=\pi R=R \pi, R / P$ is a finite field $\mathbf{F}_{\mathbf{p}^{\mathrm{n}}}$ consisting of $p^{n}$ elements for some $n \in \mathbb{N}[30], \bmod _{\mathbf{K}}(\pi):=q^{-1}$ and $\Gamma_{\mathbf{K}}:=\bmod _{\mathbf{K}}(\mathbf{K})$, where $\bmod _{\mathbf{K}}$ is the modular function of $\mathbf{K}$ associated with the nonnegative Haar measure $\mu$ on $\mathbf{K}$ such that $\mu(x S)=\bmod _{\mathbf{K}}(x) \mu(S)$ for each $0 \neq x \in \mathbf{K}, \bmod _{\mathbf{K}}(0):=0$ and each Borel subset $S$ in $\mathbf{K}$ with $\mu(S)<\infty, P:=\{x \in \mathbf{K}:|x|<1\}, R:=B(\mathbf{K}, 0,1)$. Then each $x \in \mathbf{K}$ can be written in the form $x=\sum_{l} x_{l} \pi^{l}$, where $x_{l} \in\left\{0, \theta_{1}, \ldots, \theta_{p^{n}-1}\right\}, \min _{x_{l} \neq 0} l=:-\operatorname{ord}_{\mathbf{K}}(x)>-\infty, \theta_{0}+P$, $\theta_{1}+P, \ldots, \theta_{p^{n}-1}+P$ is the disjoint covering of $R, \theta_{0}:=0$. Consider in $\mathbf{K}$ the linear ordering $a \triangle b$ if $a_{k}=b_{k}, \ldots, a_{s}=b_{s}, a_{s+1}<b_{s+1}$, where $a, b \in \mathbf{K}$, by our definition $\theta_{s}<\theta_{v}$ for each $s<v, k:=\min \left(\operatorname{ord}_{\mathbf{K}}(a), \operatorname{ord}_{\mathbf{K}}(b)\right)$. In $B(\mathbf{K}, 0,1)$ the largest element relative to such linear ordering is $\beta:=\sum_{l=0}^{\infty} \theta_{\left(p^{n}-1\right)} \pi^{l}=\theta_{\left(p^{n}-1\right)} /(1-\pi)$.

Although this linear ordering is preserved neither by additive nor by multiplicative structures of $\mathbf{K}$, it is useful (see, e.g., [26, 27] and [22, Section 62]).

2.2.2. Let $v_{0}, \ldots, v_{k} \in \mathbf{K}(\alpha)^{m}$ such that vectors $v_{1}-v_{0}, \ldots, v_{k}-v_{0}$ are K-linearly independent, then the subset $s:=\left[v_{0}, \ldots, v_{k}\right]:=\left\{z \in \mathbf{K}(\alpha)^{m}: z=a_{0} v_{0}+\cdots+a_{k} v_{k} ; a_{0}+\cdots+\right.$ $\left.a_{k}=1 ; a_{0}, \ldots, a_{k} \in B(\mathbf{K}, 0,1)\right\}$ is called the simplex of dimension $k$ over $\mathbf{K}, k=\operatorname{dim}_{\mathbf{K}} s$. A polyhedron $P$ is by our definition the union of a locally finite family $\Psi_{P}$ of simplexes. For compact $P$ a family $\Psi_{P}$ can be chosen finite. An oriented $k$-dimensional simplex is a simplex together with a class of linear orderings of its vertices $v_{0}, \ldots, v_{k}$. Two linear orderings are equivalent if they differ in an even transposition of vertices. For a simplicial complex $S$ let $C_{q}(S)$ be an Abelian group generated by simplices $s^{q}$ of dimension $q$ over $\mathbf{K}$ and relations $s_{1}^{q}+s_{2}^{q}=0$, if $s_{1}^{q}$ and $s_{2}^{q}$ are differently oriented simplices (see the real case in $\left[25\right.$, Chapter 4]). Then there exists the homomorphism $\partial_{q}: C_{q}(S) \rightarrow C_{q-1}(S)$ such that $\partial_{q}\left[v_{0}, \ldots, v_{q}\right]:=\sum_{l=0}^{q}(-1)^{l}\left[v_{0}, \ldots, v_{l-1}, v_{l+1}, \ldots, v_{q}\right]$ and $\partial_{q}\left[v_{0}, \ldots, v_{q}\right]$ is called the oriented K-boundary of $s^{q}$.

2.2.3. A clopen compact subset $\Omega$ in $(\mathbf{K} \oplus \alpha \mathbf{K})^{m}$ is totally disconnected and its topological boundary is empty. Nevertheless, using the following affine construction it is possible to introduce convention about certain curves and boundaries which will serve for the antiderivation operators.

Let $\Omega$ be a locally $\mathbf{K}$-convex subset in $\mathbf{K}(\alpha)^{m}$ for which there exists a sequence $\Omega_{n}$ of polyhedra with $\Omega_{n} \subset \Omega_{n+1}$ for each $n \in \mathbb{N}, \Omega=\operatorname{cl}\left(\bigcup_{n} \Omega_{n}\right)$, where $\operatorname{cl}(S)$ denotes the closure of a subset $S$ in $\mathbf{K}(\alpha)^{m}$. Suppose each $\Omega_{n}$ is the union of simplices $s_{j, n}$ with vertices $v_{0, n}^{j}, \ldots, v_{k, n}^{j}, j=1, \ldots, b(n) \in \mathbb{N} ;$ moreover, $\operatorname{dim}_{\mathbf{K}}\left(s_{j, n} \cap s_{j^{\prime}, n}\right)<k$ for each $j \neq j^{\prime}$ and each $n$, where $k>0$ is fixed. Then define the oriented K-border $\partial \Omega_{n}:=\sum_{j, l}(-1)^{l}\left[v_{0, n}^{j}, \ldots, v_{l-1, n}^{j}\right.$, $\left.v_{l+1, n}^{j}, \ldots, v_{k, n}^{j}\right]$. Consider $\Omega_{n}$ for each $n$ such that if $\operatorname{dim}_{\mathbf{K}}\left(s_{j, n} \cap s_{j^{\prime}, n}\right)=k-1$ for some $j \neq$ $j^{\prime}$, then $s_{j, n} \cap s_{j^{\prime}, n}=\left[v_{0, n}^{j}, \ldots, v_{l-1, n}^{j}, v_{l+1, n}^{j}, \ldots, v_{k, n}^{j}\right]=\left[v_{0, n}^{j^{\prime}}, \ldots, v_{l^{\prime}-1, n}^{j^{\prime}}, v_{l^{\prime}+1, n}^{j^{\prime}}, \ldots, v_{k, n}^{j^{\prime}}\right]$ and $\left(l-l^{\prime}\right)$ is odd. For each $n$ choose a set of vertices generating $\Omega_{n}$ of minimal cardinality and such that the sequence $\left\{\partial \Omega_{n}: n\right\}$ converges relative to the distance function $d(S, B):=$ $\max \left(\sup _{x \in S} \rho(x, B), \sup _{b \in B} \rho(b, S)\right)$, where $\rho(x, B):=\inf _{b \in B} \rho(x, b)$ and $\rho(x, b):=|x-b|$. Then by our definition $\partial \Omega:=\lim _{n \rightarrow \infty} \partial \Omega_{n}$. 
Evidently, each clopen compact subset $\Omega$ has such decomposition into simplices and the described $\partial \Omega$, since $\Omega$ is the finite union of balls, but for two balls $B_{1}$ and $B_{2}$ in $(\mathbf{K} \oplus \alpha \mathbf{K})^{m}$ either $B_{1} \subset B_{2}$ or $B_{2} \subset B_{1}$ or $B_{1} \cap B_{2}=\varnothing$ due to the ultrametric inequality and each ball $B$ has such decomposition into simplices as described above.

\subsubsection{We say that a subset $\Omega$ in $A(\mathbf{K} \oplus \alpha \mathbf{K})^{m}$ encompasses a point $z$ if $z \in \Omega$.}

For the unit ball relative to the metric $\rho\left(z_{1}, z_{2}\right):=\left|z_{1}-z_{2}\right|$, let its non-Archimedean canonical oriented $\mathbf{K}$-border $\partial_{c} B_{\rho}(\mathbf{K} \oplus \alpha \mathbf{K}, 0,1)$ be given by the set $[(-\beta,-\beta),(\beta,-\beta)] \cup$ $[(\beta,-\beta),(\beta, \beta)] \cup[(\beta, \beta),(-\beta, \beta)] \cup[(-\beta, \beta),(-\beta,-\beta)]$, where $[a, b]:=\{z \in \mathbf{K} \oplus \alpha \mathbf{K}: z=$ $(1-t / \beta) a+(t / \beta) b, t \in B(\mathbf{K}, 0,1)\}$ for each $a, b \in \mathbf{K} \oplus \alpha \mathbf{K}$. Then $\partial_{c} B\left((\mathbf{K} \oplus \alpha \mathbf{K})^{m}, 0,1\right):=$ $\bigcup_{l=1}^{m} B(\mathbf{K} \oplus \alpha \mathbf{K}, 0,1)^{l-1} \times \partial_{c} B(\mathbf{K} \oplus \alpha \mathbf{K}, 0,1) \times B(\mathbf{K} \oplus \alpha \mathbf{K}, 0,1)^{m-l}, \partial_{c} B\left((\mathbf{K} \oplus \alpha \mathbf{K})^{m}, z, q^{k}\right):=$ $z+\pi^{-k} \partial_{c} B\left((\mathbf{K} \oplus \alpha \mathbf{K})^{m}, 0,1\right)$. This is the particular case of Section 2.2.3.

A continuous mapping $\gamma: B(\mathbf{K}, 0,1) \rightarrow A(\mathbf{K}(\alpha))^{m}$ is called a path. We say that $\gamma$ encompasses a point $z \in A(\mathbf{K}(\alpha))^{m}$ if

(i) $z \in \Omega$, where $\partial \Omega=\gamma, \operatorname{dim}_{\mathbf{K}} \Omega=2$,

(ii) $z \notin \gamma(B(\mathbf{K}, 0,1))$,

(iii) $|z|<\sup _{\theta \in B(\mathbf{K}, 0,1)}|\gamma(\theta)|$ for $z \neq A$, $\sup _{\theta \in B(\mathbf{K}, 0,1)}|\gamma(\theta)|<\infty$ for $z=A$.

A path $\gamma$ is called locally affine if there exists a finite partition $\mathscr{L}$ of $\gamma(B(\mathbf{K}, 0,1))$ such that $\gamma=\bigcup_{l=1}^{n} \tau_{l}$, where $\mathscr{E}:=\left\{z_{0}, z_{1}, \ldots, z_{n}\right\}, \tau_{l}:=\left[z_{l-1}, z_{l}\right]$ for each $l=1, \ldots, n$. We consider the family $\mathscr{F}_{q}$ of all paths $\gamma$ for which there exists a sequence $\left\{\gamma_{n}: n\right\} \subset \mathscr{F}_{a}$ converging relative to the distance function $d^{\prime}(S, B):=\max \left(\sup _{x \in S} \rho^{\prime}(x, B), \sup _{b \in B} \rho^{\prime}(b, S)\right)$ to $\gamma$ in $\left(A(\mathbf{K}(\alpha))^{m}, \rho^{\prime}\right)$ and such that there exists a homeomorphism $\nu$ of $\gamma(B(\mathbf{K}, 0,1))$ with $B(\mathbf{K}, 0,1)$ and $\nu$ is a piecewise ${ }_{P} C^{q+1}$-diffeomorphism with it, where $\mathscr{F}_{a}$ denotes the family of all locally affine paths, $q \in \mathbb{N}$. In addition, we take $\Omega$ and $\gamma$ such that $\gamma=\partial \Omega$ in accordance with Section 2.2.3.

Since $A \mathbf{K}(\alpha)^{m}$ and $A(\mathbf{K} \oplus \alpha \mathbf{K})^{m}$ are compact, then a clopen compact set $\Omega$ in $A \mathbf{K}(\alpha)^{m}$ or in $A(\mathbf{K} \oplus \alpha \mathbf{K})^{m}$ is homeomorphic with a clopen compact subset $\kappa(\Omega)$ in $\mathbf{K}(\alpha)^{m}$ or $(\mathbf{K} \oplus$ $\alpha \mathbf{K})^{m}$, respectively (see [5, Theorem 6.2.16 and Corollary 6.2.17] about universality of the Cantor cube for zero-dimensional spaces), where $\kappa: \Omega \rightarrow \kappa(\Omega)$ is the homeomorphism. Therefore, we can consider ${ }_{\Omega} P^{n}, \partial \Omega$, and ${ }_{\partial \Omega} P^{n}$ induced by $\kappa$ of such sets $\Omega$ also.

2.2.5. Let $M$ be a $C^{\xi+(1,0)}$-manifold of dimension $k$ over $\mathbf{K}$ such that $\xi=(q, n-1)$, where spaces $C^{\xi}\left(\mathbf{K}^{a}, \mathbf{K}^{b}\right):=C\left(\xi, \mathbf{K}^{a} \rightarrow \mathbf{K}^{b}\right)$ and $C^{\xi}$-manifolds and uniform spaces $C^{\xi}(M, N)$ of all $C^{\xi}$-mappings $f: M \rightarrow N$ were defined in [15, Section I.2.4] and [16] $0 \leq q \in \mathbb{Z}, 0<n \in \mathbb{Z}$, and

$$
\begin{aligned}
& { }_{P} C_{0}^{\xi+(0,1)}\left(\Omega, \mathbf{L}^{b}\right):=P^{n}\left(C^{\xi}\left(\Omega, \mathbf{L}^{b}\right)\right), \\
& { }_{P} C^{\xi+(0,1)}\left(\Omega, \mathbf{L}^{b}\right):={ }_{P} C_{0}^{\xi+(0,1)}\left(\Omega, \mathbf{L}^{b}\right) \oplus \mathbf{L}^{b}
\end{aligned}
$$

were described in [19, Lemma 2.1], where $P^{n}:={ }_{\Omega} P^{n}$ (see Section 2.1).

Suppose that charts $\left(V_{j}, \phi_{j}\right)$ of the atlas $A t(M)$ of $M$ are such that

( $\alpha) V_{j}$ are clopen in $M$,

(ß) $\bigcup_{j} V_{j}=M$,

( $\gamma) \phi_{j}: V_{j} \rightarrow \phi_{j}\left(V_{j}\right) \subset U^{k}$ are homeomorphisms on clopen subsets in $U^{k}$, where 
$(\delta) \phi_{i, j}:=\phi_{i} \circ \phi_{j}^{-1} \in{ }_{P, x_{l}} C^{\xi+(1,0)} \cap C^{\xi}\left(W_{i, j}, \mathbf{K}^{k}\right)$ for each $i \neq j$ with $V_{i} \cap V_{j} \neq \varnothing$ and each coordinate $x_{l}$ induced from $\mathbf{K}, l=1, \ldots, k, W_{i, j}:=\operatorname{dom}\left(\phi_{i, j}\right)$,

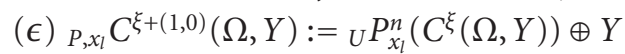

for a Banach space $Y$ over $\mathbf{L}, \mathbf{K} \subset \mathbf{L}$. Thus put

$$
\begin{aligned}
& { }_{S} C^{\xi+(1,0)}(\Omega, Y) \\
& \quad:=\left\{f \in C^{\xi+(1,0)}(\Omega, Y): f\left(x_{1}, \ldots, x_{k}\right) \in{ }_{P, x_{l}} C^{\xi+(1,0)}(\Omega, Y) \text { for each } l=1, \ldots, k\right\},
\end{aligned}
$$

where $Y$ is a Banach space over $\mathbf{L}$. In particular, ${ }_{S} C^{\xi+(1,0)}(U, Y)={ }_{P} C^{\xi+(0,1)}(U, Y)=$ ${ }_{P} C^{q+n}(U, Y)$, but for $\operatorname{dim}_{K} \Omega>1$ these spaces are different ${ }_{S} C^{\xi+(1,0)}(\Omega, Y) \neq{ }_{P} C^{\xi+(0,1)}(\Omega, Y)$.

Then we call $M$ satisfying conditions $(\alpha-\epsilon)$ the ${ }_{S} C^{\xi+(1,0)}$-manifold, and such mappings $\phi_{i, j}$ are called the ${ }_{S} C^{\xi+(1,0)}$-mappings.

Tensor fields over $M$ were defined in [19, Sections 2.1.2 and 2.1.3]. Then the bundle of $r$-differential forms is the antisymmetrized bundle $\psi_{r}: \Lambda^{r} M \rightarrow M$ of the bundle $\tau_{r}$ : $T_{r} M \rightarrow M$ of $r$-fold covariant tensors.

A mapping $\phi: V \rightarrow W$ of a (cl)open subset $V$ on a (cl)open subset $W$ of $\mathbf{K}^{k}$ is called a ${ }_{S} C^{\xi+(1,0)}$-diffeomorphism if $\phi$ is surjective and bijective with $\phi \in{ }_{S} C^{\xi+(1,0)}\left(V, \mathbf{K}^{k}\right)$ and $\phi^{-1} \in{ }_{S} C^{\xi+(1,0)}\left(W, \mathbf{K}^{k}\right)$.

Consider the ${ }_{S} C^{\xi+(1,0)}$-diffeomorphism $\phi: \eta \rightarrow \phi(\eta)$, where

(i) $\eta=\left[v_{0}, v_{1}\right] \times\left[v_{1}, v_{2}\right] \times \cdots \times\left[v_{k-1}, v_{k}\right]$

is the parallelepiped in $\mathbf{K}^{k}$, vectors $v_{1}-v_{0}, \ldots, v_{k}-v_{0}$ are $\mathbf{K}$-linearly independent. Then for a $k$-differential $C^{(0, n-1)}$-form $w$ on $\phi(\eta)$ define

(1) $\phi(\eta) P^{n} w:={ }_{\eta} P^{n} \phi^{*} w$,

where $\phi^{*} w$ is the pullback of $w$ defined in local coordinates in the standard way, since $\phi$ is the ${ }_{S} C^{\xi+(1,0)}$-diffeomorphism such that

(2) $\phi(\eta) P^{n} w=0$ for $\operatorname{dim}_{\mathbf{K}} \eta \neq k$,

since $w=0$ for $k>\operatorname{dim}_{K} M$. Without loss of generality, take $0 \in U$ and $\sigma_{0}(0)=0$, then $\sigma_{l}(0)=0$ for each $l \in \mathbb{N}$, consequently, $\left.U^{n}\right|_{\{0\}}=0$. Therefore, $\left.U^{m} P^{n}\right|_{\left(U^{m} \cap \mathbf{K}^{k} \times\{0\}^{m-k}\right)} w=0$ for $k<\operatorname{dim}_{\mathrm{K}} \Omega=m$. Each such parallelepiped $\eta$ is the finite union of simplices satisfying conditions of Section 2.2.3. The orientation of $\partial \eta$ is induced by the orientations constituting its simplices which are consistent. Consider such parallelepipeds $\eta_{j, q, l}$ with $l=1, \ldots, b(q) \in \mathbb{N}$ and

(ii) $\operatorname{dim}_{\mathbf{K}}\left(\eta_{j, q, l} \cap \eta_{j, q, l^{\prime}}\right)<k$ for each $l \neq l^{\prime}$,

(iii) $\operatorname{cl}\left(\bigcup_{q} \kappa_{j, q}\right)=\phi_{j}\left(V_{j}\right)$, where

(iv) $\bigcup_{l=1}^{b(q)} \eta_{j, q, l}=: \kappa_{j, q}$,

(v) $\lim _{q \rightarrow \infty} \max _{l} \operatorname{diam}\left(\eta_{j, q, l}\right)=0$.

Since $_{\eta_{j, q, l}} P^{n} v+{ }_{\eta_{j, q, l^{\prime}}} P^{n} v={ }_{\eta_{j, q, l} \cup \eta_{j, q, l^{\prime}}} P^{n} v$ for each differential $C^{(0, n-1)}-k$-form $v$ with support in $U^{k}$ and each $l \neq l^{\prime}$ and $U^{k} P^{n}$ is the continuous operator from $C^{(0, n-1)}\left(U^{k}, \mathbf{L}\right)$ to $C^{(0, n)}\left(U^{k}, \mathbf{L}\right)$, then there exists

(vi) $\lim _{q \rightarrow \infty} \sum_{l=1}^{b(q)} \eta_{j, q, l} P^{n} v=:_{\phi_{j}\left(V_{j}\right)} P^{n} v$. 
Using transition mappings $\phi_{i, j}$ and considering clopen disjoint covering

(vii) $W_{j}:=V_{j} \backslash \bigcup_{l=1}^{j-1} V_{j}$ of $M$,

we get

(viii) ${ }_{M} P^{n} w=\sum_{j} W_{j} P^{n} w$

independent of the choice of local coordinates in $M$. Note that since $|\beta|=1$, then $B\left(\mathbf{K}^{l}, z, r\right)$ can be represented as the parallelepiped with the $\mathbf{K}$-boundary $\partial_{c} B\left(\mathbf{K}^{l}, z, r\right)$ described above due to the ultrametric inequality. Due to (vi), (vii), and (viii), ${ }_{\gamma} P^{n} v$ is defined for locally affine path $\gamma$ (see Section 2.2.4), which is the ${ }_{P} C^{n}$-manifold that will be supposed henceforth.

Each compact manifold $M$ has a finite dimension over $\mathbf{K}$ and using $W_{j}$ we get an embedding into $\mathbf{K}^{b}$ for some $b \in \mathbf{K}$. Let $\phi: \Omega \rightarrow M$ be such that $\phi$ is surjective and bijective, $\phi$ and $\phi^{-1} \in{ }_{S} C^{\xi+(1,0)}$, which means that $\phi_{j} \circ \phi \in{ }_{S} C^{\xi+(1,0)}\left(\phi^{-1}\left(V_{j}\right), \mathbf{K}^{k}\right)$ and $\phi^{-1} \circ \phi_{j}^{-1} \in{ }_{s} C^{\xi+(1,0)}\left(\phi_{j}\left(V_{j}\right), \mathbf{K}^{k}\right)$ for each $j$, where $\phi^{-1}(M)=\Omega \subset U^{k}$ satisfies conditions of Section 2.2.3, At $(M)=\left\{\left(V_{j}, \phi_{j}\right): j\right\}$ is an atlas of a ${ }_{S} C^{\xi+(1,0)}$-manifold $M$ (see above). Such $\phi$ is called the ${ }_{S} C^{\xi+(1,0)}$-diffeomorphism of $\Omega$ onto $M$. Then $M$ is oriented together with $\Omega$. Thus $\partial M:=\phi(\partial \Omega)$ is the oriented boundary. We can also consider the analytic manifold $M$ and the analytic diffeomorphism $\phi$. Each compact $C^{\xi}$-manifold $M$ can be supplied with the analytic manifold structure using a disjoint covering refined into At $(M)$.

Theorem 2.1. Let $M$ be a compact ${ }_{S} C^{\xi}$ - or ${ }_{P} C^{\xi}$-manifold over the local field $\mathbf{K}$ with dimension $\operatorname{dim}_{\mathbf{K}} M=k$ and an atlas $A t(M)=\left\{\left(V_{j}, \phi_{j}\right): j=1, \ldots, n\right\}$, where $\xi=(q, n), 1 \leq q \in$ $\mathbb{N}, 0 \leq n \in \mathbb{Z}$, then there exists a ${ }_{S} C^{\xi}$ - or ${ }_{P} C^{\xi}$-embedding of $M$ into $\mathbf{K}^{\text {nk }}$, respectively.

Proof. Let $\left(V_{j}, \phi_{j}\right)$ be the chart of the atlas $A t(M)$, where $V_{j}$ is clopen in $M$, hence $M \backslash V_{j}$ is clopen in $M$. Therefore, there exists a ${ }_{S} C^{\xi}$ - or ${ }_{P} C^{\xi}$-mapping $\psi_{j}$ of $M$ into $\mathbf{K}^{k}$ such that $\psi_{j}\left(M \backslash V_{j}\right)=\left\{x_{j}\right\}$ is the singleton and $\psi_{j}: V_{j} \rightarrow \psi_{j}\left(V_{j}\right)$ is the ${ }_{S} C^{\xi}$ - or ${ }_{P} C^{\xi}$-diffeomorphism onto the clopen subset $\psi_{j}\left(V_{j}\right)$ in $\mathbf{K}^{k}$, correspondingly, $x_{j} \in \mathbf{K}^{k} \backslash \psi_{j}\left(V_{j}\right)$, since the operator ${ }_{M} P^{n}$ is K-linear, ${ }_{M} P^{n} 0=0$ and the covering $\left\{V_{j}: j\right\}$ of $M$ has a disjoint finite refinement $\left\{W_{k}: k\right\}$ such that $P_{x_{l}}^{n}[f]=P_{x_{l}}^{n}\left[\sum_{k} f \chi_{W_{k}}\right]=\sum_{k} P_{x_{l}}^{n}\left[f \chi_{W_{k}}\right]$ for each $f \in C^{(q, n-1)}(M, \mathbf{K})$ and each coordinate $x_{l}$ (see Sections 2.1 and 2.2.5). Then the mapping $\psi(z):=\left(\psi_{1}(z), \ldots\right.$, $\left.\psi_{n}(z)\right)$ is the embedding into $\mathbf{K}^{n k}$, since the rank is such that $\operatorname{rank}\left[d_{z} \psi(z)\right]=k$ at each point $z \in M$, because $\operatorname{rank}\left[d_{z} \psi_{j}(z)\right]=k$ for each $z \in V_{j}$ and $\operatorname{dim}_{\mathbf{K}} \psi\left(V_{j}\right) \leq \operatorname{dim}_{\mathbf{K}} M=k$. Moreover, $\psi(z) \neq \psi(y)$ for each $z \neq y \in V_{j}$, since $\psi_{j}(z) \neq \psi_{j}(y)$. If $z \in V_{j}$ and $y \in M \backslash V_{j}$, then there exists $l \neq j$ such that $y \in V_{l} \backslash V_{j}, \psi_{j}(z) \neq \psi_{j}(y)=x_{j}$.

Theorem 2.2. Let $M$ be a compact oriented manifold over $\mathbf{K}$ of dimension $\operatorname{dim}_{\mathbf{K}} M=k>0$ with an oriented boundary $\partial M$ and let $w$ be a differential $(k-1)$-form as in Section 2.2.5 such that its pullback $\phi^{*} w$ is a differential $(k-1){ }_{S} C^{(1, n-1)}$-form, then

$$
{ }_{M} P^{n} d w={ }_{\partial M} P^{n} w
$$

Proof. Since $M$ is the manifold of $\operatorname{dim}_{\mathrm{K}} M=k>0$, then $M$ is dense in itself and compact, hence $\Omega$ is dense in itself and compact (see [5, Chapter 1 and Theorems 3.1.2 and 3.1.10]) and the approximation of the identity can be applied to $\Omega$. In view of formulas (2.1)-(2.4) 
and (1), (2) on the space of $C^{\xi}$-differential forms, operators ${ }_{U} P_{x_{q}}^{n}$ and ${ }_{U} P_{x_{s}}^{n}$ commute for each $1 \leq q, s \leq k$. Then

(i) $\left.{ }_{U} P^{n} f\right|_{a} ^{b}=-\left._{U} P^{n} f\right|_{b} ^{a}$, where $\left.{ }_{U} P^{n} f\right|_{a} ^{b}:={ }_{U} P^{n} f(b)-{ }_{U} P^{n} f(a)$.

In view of conditions imposed on the manifold $M$, partitions of $\Omega_{n}$ into unions of parallelepipeds, which are finite unions of simplices as in Section 2, formula (i), and (1), also using the limit (vi) and formula (viii), it is sufficient to verify (2.1) for a parallelepiped and an arbitrary term $\psi:=f(z) d z_{1} \wedge \cdots \wedge d z_{q-1} \wedge d z_{q+1} \wedge \cdots \wedge d z_{k}$ corresponding to the differential $(k-1)_{S} C^{(1, n-1)}$-form $\phi^{*} w$. Consider in $\mathbf{K}^{k}$ the standard orthonormal base $e_{1}, \ldots, e_{k}$, where $e_{l}:=(0, \ldots, 0,1,0, \ldots, 0)$ is the vector with 1 in $l$ th place. Without loss of generality, using limits we can take the parallelepipeds $\tau=\left[v_{0}, v_{1}\right] \times$ $\cdots \times\left[v_{k-1}, v_{k}\right]$ with $v_{l}-v_{l-1}=\lambda_{l} e_{l}$ for each $l=1, \ldots, k$, where $0 \neq \lambda_{l} \in \mathbf{K}$. Therefore, $d f(z)=(-1)^{q-1}\left(\partial f(z) / \partial z_{q}\right) d z_{1} \wedge \cdots \wedge d z_{k}$. Since $f \in{ }_{s} C^{(1, n-1)}$, then $\left.{ }_{U} P_{z_{q}}^{n}\left(\partial f(z) / \partial z_{q}\right)\right|_{a} ^{b}$ $=f\left(z_{1}, \ldots, z_{q-1}, b, z_{q+1}, \ldots, z_{k}\right)-f\left(z_{1}, \ldots, z_{q-1}, a, z_{q+1}, \ldots, z_{k}\right)$ for each $l=1, \ldots, k$. Consequently,

(ii)

$$
\begin{aligned}
{ }_{\tau} P^{n} d \psi= & (-1)^{q-1}{ }_{\left[v_{0}, v_{1}\right] \times \cdots \times\left[v_{q-2}, v_{q-1}\right] \times\left[v_{q+1}, v_{q+2}\right] \times \cdots \times\left[v_{k-1}, v_{k}\right]} P^{n} d z_{1} \\
& \wedge \cdots \wedge d z_{q-1} \wedge d z_{q+1} \wedge \cdots \wedge d z_{k\left[v_{q-1}, v_{q}\right]} P^{n}\left(\frac{\partial f(z)}{\partial z_{q}}\right) d z_{q} \\
= & (-1)^{q-1}{ }_{\left[v_{0}, v_{1}\right] \times \cdots \times\left[v_{q-2}, v_{q-1}\right] \times\left[v_{q+1}, v_{q+2}\right] \times \cdots \times\left[v_{k-1}, v_{k}\right]} \\
& \times P^{n}\left\{f\left(z_{1}, \ldots, z_{q-1}, v_{q}, d z_{q+1}, \ldots, d z_{k}\right)\right. \\
& \left.\quad-f\left(z_{1}, \ldots, z_{q-1}, v_{q-1}, d z_{q+1}, \ldots, d z_{k}\right)\right\} d z_{1} \\
& \wedge \cdots \wedge d z_{q-1} \wedge d z_{q+1} \wedge \cdots \wedge d z_{k}
\end{aligned}
$$

for each $q=1, \ldots, k$. In view of (2) antiderivations of $\psi$ by other pieces $(-1)^{s-1}\left[v_{0}, v_{1}\right] \times$ $\cdots \times\left[v_{s-2}, v_{s-1}\right] \times\left(\left\{v_{s}\right\}-\left\{v_{s-1}\right\}\right) \times\left[v_{s}, v_{s+1}\right] \times \cdots \times\left[v_{k-1}, v_{k}\right]$ corresponding to $s \neq q$ of the $\mathrm{K}$-border are zero.

Corollary 2.3. Let $M$ be a compact oriented manifold over $\mathbf{K}$ of dimension $\operatorname{dim}_{\mathbf{K}} M=$ $k>0$ with an oriented boundary $\partial M$ and let $w$ be a differential $(k-1) C^{(1, n-1)}$-form as in Section 2.2.5 such that its pullback $\phi^{*} w=\sum_{j_{1}<\cdots<j_{k-1}} f_{j_{1}, \ldots, j_{k-1}} d z_{j_{1}} \wedge \cdots \wedge d z_{j_{k-1}}$ has each function $f_{j_{1}, \ldots, j_{k-1}}$ in ${ }_{P, z_{j}} C^{n}(U, \mathbf{L})$ by the variable $z_{j}$ for each $j$ such that $j \in\{1, \ldots, k\} \backslash$ $\left\{j_{1}, \ldots, j_{k-1}\right\}$, then

$$
{ }_{M} P^{n} d w={ }_{\partial M} P^{n} w .
$$

Proof. Repeating the proof of Theorem 2.2 for each term $f_{j_{1}, \ldots, j_{k-1}} d z_{j_{1}} \wedge \cdots \wedge d z_{j_{k-1}}$ of $w$ and applying (i), (ii), we get the statement of this corollary.

2.2.6. Remarks and notations. Let $f \in C^{1}(\mathbf{K}(\alpha), Y)$, where $Y$ is a Banach space over $\mathbf{L}$, $\mathbf{L}$ is a field containing $\mathbf{K}(\alpha)$ such that $\mathbf{L}$ is complete relative to its uniformity, and the multiplicative norm in $\mathbf{L}$ is the extension of the multiplicative norm in $\mathbf{K}(\alpha)$. As the Banach space $\mathbf{K}(\alpha)$ over $\mathbf{K}$ is isomorphic with $\mathbf{K}^{r}$, where $2 \leq r \in \mathbb{N}$ consider such structure of $\mathbf{K}(\alpha)$ over $\mathbf{K}$. That is, $\mathbf{K}(\alpha)$ over $\mathbf{K}$ is considered as $\bigoplus_{m=0}^{r-1} \alpha^{m} \mathbf{K}$, where $\alpha^{0}=1$. Thus 
each $z \in \mathbf{K}(\alpha)$ has the unique decomposition $z=x+\alpha y$ with $x \in \mathbf{K}$ and $y \in \bigoplus_{m=0}^{r-2} \alpha^{m} \mathbf{K}$. Henceforth, either we consider $r=2$ which implies that in the decomposition $z=x+\alpha y$ of $z \in \mathbf{K}(\alpha)$ both $x$ and $y \in \mathbf{K}$, or we consider $\mathbf{K} \oplus \alpha \mathbf{K}$ for $r>2$ such that again each $z \in \mathbf{K} \oplus \alpha \mathbf{K}$ has the unique decomposition $z=x+\alpha y$ with both $x$ and $y \in \mathbf{K}$. Only these two variants with $\mathbf{K} \oplus \alpha \mathbf{K}$ will be considered below if nothing else is specified (see also a justification of this in Remark 2.10).

Then we write each $\zeta \in \mathbf{K}(\alpha)$ in the form $\zeta=x+\alpha y$, where $x \in \mathbf{K}, y \in \bigoplus_{m=0}^{r-2} \alpha^{m} \mathbf{K}, \alpha$ for $\mathbf{K}$ is chosen as in Section 2.1. Denote by $\bar{\zeta}:=x-\alpha y$ the so-called conjugate element to $\zeta$. Then $x=(\zeta+\bar{\zeta}) / 2$ and $y=(\zeta-\bar{\zeta}) /(2 \alpha)$. Therefore,

(i) $\partial f(\zeta, \bar{\zeta}) / \partial x=\partial f(\zeta, \bar{\zeta}) / \partial \zeta+\partial f(\zeta, \bar{\zeta}) / \partial \bar{\zeta}$ and

(ii) $\partial f(\zeta, \bar{\zeta}) / \partial y=\alpha \partial f(\zeta, \bar{\zeta}) / \partial \zeta-\alpha \partial f(\zeta, \bar{\zeta}) / \partial \bar{\zeta}$, consequently,

(iii) $\partial f(\zeta, \bar{\zeta}) / \partial \zeta=\left[\partial f(\zeta, \bar{\zeta}) / \partial x+\alpha^{-1} \partial f(\zeta, \bar{\zeta}) / \partial y\right] / 2$ and

(iv) $\partial f(\zeta, \bar{\zeta}) / \partial \bar{\zeta}=\left[\partial f(\zeta, \bar{\zeta}) / \partial x-\alpha^{-1} \partial f(\zeta, \bar{\zeta}) / \partial y\right] / 2$.

In particular, the external differentiation of differential $C^{1}$-forms $w$ on a clopen subset $\Omega$ in $(\mathbf{K} \oplus \alpha \mathbf{K})^{m}$ has the form

(v) $d w=\partial w+\bar{\partial} w$, where

(vi) $w=\sum_{I, J} w_{I, J}(\zeta, \bar{\zeta}) d \zeta^{\wedge I} \wedge d \bar{\zeta} \wedge$,

(vii) $\partial w=\sum_{I, J, l}\left(\partial w_{I, J} / \partial \zeta_{l}\right) d \zeta_{l} \wedge d \zeta^{I} \wedge d \bar{\zeta}^{\wedge J}$,

(viii) $\bar{\partial} w=(-1)^{|I|} \sum_{I, J, l}\left(\partial w_{I, J} / \partial \bar{\zeta}_{l}\right) d z^{I} \wedge d \bar{\zeta}^{l} \wedge d \bar{\zeta}^{\wedge J}$, where $d \zeta^{\wedge I}:=d \zeta_{I_{1}} \wedge \cdots \wedge d \zeta_{I_{b}}$, $d \bar{\zeta}^{\wedge J}:=d \bar{\zeta}_{J_{1}} \wedge \cdots \wedge d \bar{\zeta}_{J_{c}}, 1 \leq I_{1}<\cdots<I_{b} \leq m, 1 \leq J_{1}<\cdots<J_{c} \leq m$,

such that $w$ is the $(b, c)$-form with coefficients $w_{I, J} \in C^{1}(\Omega, Y),|I|:=b$.

If $r>2$, then the differential $s$-form $w$ can be written as

(ix) $w=\sum_{J,|J|=s} w_{J} d z^{\wedge J}, z=\left(z_{1}, \ldots, z_{r m}\right), z_{l} \in \mathbf{K}$, for each $l=1, \ldots, r m, d z^{\wedge J}:=d z_{J_{1}} \wedge$ $\cdots \wedge d z_{J_{s}}, 1 \leq J_{1}<\cdots<J_{s} \leq r m$. Let $\Lambda\left(K(\alpha)^{m}\right)$ denote the Grassmann algebra (exterior algebra) of $\mathbf{K}(\alpha)^{m}$, where $\mathbf{K}(\alpha)$ is considered as a $\mathbf{K}$-linear space, $\Lambda\left(\mathbf{K}(\alpha)^{m}\right)=\bigoplus_{l=0}^{r m} \Lambda^{l}\left(\mathbf{K}(\alpha)^{m}\right)$. Then $w \in C^{\xi}\left(\Omega, L\left(\Lambda\left(\mathbf{K}(\alpha)^{m}\right), Y\right)\right)$ is the differential form, since the space $\left(\mathbf{K}(\alpha)^{m}\right)^{*}$ of $\mathbf{K}$-linear functionals on $\mathbf{K}(\alpha)^{m}$ is the space isomorphic with $\mathbf{K}(\alpha)^{m}$ due to discreteness of $\Gamma_{\mathbf{K}}$, where $L\left(\Lambda\left(\mathbf{K}(\alpha)^{m}\right), Y\right)$ is the Banach space of $\mathbf{K}$-linear operators from $\Lambda\left(\mathbf{K}(\alpha)^{m}\right)$ into $Y$.

Henceforth, if on a manifold $M$ functions $f$ will be considered having the property $\bar{\partial} f=0$, then it will be supposed that $\bar{\partial} \phi_{i, j}=0$ for each transition mapping $\phi_{i, j}$, if another is not specified.

Consider $\omega$ such that $\omega \subset E$, where $E:=\left\{z \in \mathbf{K}(\alpha):|z|<p^{1 /(1-p)}\right\}$, since exp is the bijective analytic function on $E$, therefore we put

(x) $\exp (\omega)=\Omega$, that is, $\omega=\log (\Omega)$ for $\Omega \subset 1+E$, where exp : $E \rightarrow(1+E)$ and $\log$ : $(1+E) \rightarrow E$ are defined with the help of standard series (see [22, Sections 25 and 44]).

Consider an extension of Log. Denote $\mathbb{C}_{\mathbf{p}}^{+}:=\left\{z \in \mathbb{C}_{p}:|z-1|<1\right\}$ and $\mathbf{K}(\alpha)^{+}:=$ $\mathbf{K}(\alpha) \cap \mathbb{C}_{\mathbf{p}}^{+}$. Then $\mathbf{K}(\alpha)^{+}$is the Abelian subgroup in the additive group $\mathbb{C}_{\mathbf{p}}^{+}$and $\mathbb{C}_{\mathbf{p}}^{\times}:=$ $\mathbb{C}_{\mathbf{p}} \backslash\{0\}$ is the Abelian multiplicative group. The group $\mathbb{C}_{\mathbf{p}}^{\times}$is divisible, that is, for each $y \in \mathbb{C}_{\mathbf{p}}^{\times}$and each $n \in \mathbb{N}$ there exists $x \in \mathbb{C}_{\mathbf{p}}^{\times}$such that $x^{n}=y$. Let $X$ be a proper divisible subgroup in $\mathbb{C}_{\mathbf{p}}^{\times}$such that $\mathbb{C}_{\mathbf{p}}^{+} \subset X$. Let $G$ be a subgroup generated by $X$ and $y \in \mathbb{C}_{\mathbf{p}}^{\times} \backslash X$. Suppose $y^{n} \notin X$ for each $n \in \mathbb{N}$, then for each $g \in G$ there exist unique $n \in \mathbb{Z}$ and $x \in X$ 
such that $g=y^{n} x$. Choose $z \in \mathbb{C}_{\mathbf{p}}$, then put $\log (g):=n z+\log (x)$. The second possibility is $y^{n} \in X$ for some $n \in \mathbb{N}, n>1$. For each $g \in G$ there exist unique $n \in\{0,1, \ldots, m-1\}$ and $x \in X$ such that $g=y^{n} x$, where $m:=\min _{y^{n} \in X ; n \in \mathbb{N}} n$. Since $\mathbb{C}_{\mathbf{p}}$ is divisible, there exists $z \in \mathbb{C}_{\mathbf{p}}$ such that $z_{m}=\log \left(y_{m}\right)$, therefore, define $\log (g):=n z+\log (x)$. Using Zorn's lemma we can extend $\log$ from $\mathbb{C}_{\mathbf{p}}^{+}$on $\mathbb{C}_{\mathbf{p}}^{\times}$. In particular we can consider values of $\log (i)$ and $\log (\alpha)$ using identities $\log (1)=0, i^{4}=1, \alpha^{m} \in \mathbf{K}, \alpha^{n}=1$ for some minimal $n \in \mathbb{N}$.

The field $\mathbb{C}_{\mathbf{p}}$ considered as the locally $\mathbb{Q}_{\mathbf{p}}$-convex space is of countable type over $\mathbb{Q}_{\mathbf{p}}$, where $\mathbb{Q}_{\mathbf{p}}$ is the locally compact field of $p$-adic numbers [28]. As the topological space $\mathbb{C}_{\mathbf{p}}$ is Lindelöf, there exists consequently a countable family $\left\{g_{j}: j \in \mathbb{N}\right\} \subset \mathbb{C}_{\mathbf{p}}^{\times}, g_{1}=1$, such that $\left\{g_{j} B \backslash \bigcup_{v<j}\left(q_{v} B\right): j \in \mathbb{N}\right\}$ is the disjoint clopen covering of $\mathbb{C}_{\mathbf{p}}^{\times}$, where $B:=\left\{z \in \mathbb{C}_{\mathbf{p}}\right.$ : $\left.|z-1|<p^{1 /(1-p)}\right\}$. In view of $\log (y z)=\log (y)+\log (z)$ for each $y, z \in \mathbb{C}_{\mathbf{p}}^{\times}$the function Log is completely characterized by values of $\log$ on $B \ni z$ and $\log$ on $y=q_{j}$ for each $j \in \mathbb{N}$. The restriction $\left.\log \right|_{B}=\left.\log \right|_{B}$ has only one analytic branch. It is known from [22, Section 45] that such extension Log of Log is locally analytic, hence Log is of class $C^{\infty}$. In accordance with [22, Theorem 45.9] if $f, g: \mathbb{C}_{\mathbf{p}}^{\times} \rightarrow \mathbb{C}_{\mathbf{p}}$ are two extensions of Log: $\mathbb{C}_{\mathbf{p}}^{+} \rightarrow \mathbb{C}_{\mathbf{p}}$ as above, then there exists a constant $c \in \mathbb{C}_{\mathbf{p}}$ such that $f(x)=g(x)+c \operatorname{ord}_{p}(x)$ for all $x \in \mathbb{C}_{\mathbf{p}}^{\times}$. Thus in view of [22, Theorem 45.9] we can choose an infinite family of branches of $\log$ indexed by $\mathbb{Z}$. For the sake of convenience, put $\log (0):=A$.

From the consideration above it follows that we can choose $\log (\alpha) \neq 0$ for $\mathbf{K}(\alpha)$ and $\alpha \in \mathbb{C}_{\mathbf{p}}^{\times} \backslash \mathbb{C}_{\mathbf{p}}^{+}$as in Section 2.1 for which the extension Exp of exp on $\mathbb{C}_{\mathbf{p}}$ and the extension $\log$ of Log on $\mathbb{C}_{\mathbf{p}} \backslash\{0\}$ can be chosen such that directed going (defined by going from 0 to $\beta$ in linearly ordered $B(\mathbf{K}, 0,1)$; see Section 2.2$)$ by the oriented loop $\partial_{c} B\left(\mathbf{K}(\alpha), 0, p^{-2}\right)$ changes a branch ${ }_{n} \log$ of $\log$ on 1 in the following manner: ${ }_{n+1} \log (x)-{ }_{n} \log (x)=: \delta \neq$ 0 for each $n \in \mathbb{N}$, where $\operatorname{Exp}(\delta)=1, \delta$ is independent of $n$. This is possible, since algebraically $\mathbb{C}_{\mathbf{p}}$ and $\mathbb{C}$ are isomorphic fields [10]; also, points $p^{2}(-1,-1), p^{2}(1,-1), p^{2}(1,1)$, and $p^{2}(1,-1)$ belong to $\partial_{c} B\left(\mathbf{K}(\alpha), 0, p^{-2}\right)$.

THEOREM 2.4. Let $M$ be a compact ${ }_{S} C^{(q, n)}$-manifold over $\mathbf{K}$ satisfying conditions of Sections 2.2.5 and 2.2.6 for which $\phi^{-1}(M)=\Omega \subset \mathbf{K}(\alpha)$ with a K-boundary $\gamma:=\partial M, \operatorname{dim}_{\mathbf{K}} M=$ $2,2 \leq r \in \mathbb{N}, 0 \leq q \in \mathbb{Z}, 1 \leq n \in \mathbb{N}$, then there exists a constant $0 \neq C:=C_{n}(\alpha) \in \mathbf{K}(\alpha)$, such that

$$
f(z)=C^{-1}{ }_{\partial M} P^{n}\left\{f(\zeta)(\zeta-z)^{-1} d \zeta\right\}-C^{-1}{ }_{M} P^{n}\left\{\frac{(\bar{\partial} f \wedge d \zeta)}{(\zeta-z)}\right\}
$$

for each

$$
f_{1}(z+\operatorname{Exp}(\eta))=: \psi(\eta) \in{ }_{S} C^{(1, n-1)}\left(\omega_{\epsilon}, Y\right)
$$

and each marked $z \in M$ encompassed by $\gamma$.

For each $\epsilon=\epsilon_{j}, 0<\epsilon_{j}, j \in \mathbb{N},\left\{\epsilon_{j}: j\right\}$ is a sequence in $\Gamma_{\mathbf{K}}$ with $\lim _{j \rightarrow \infty} \epsilon_{j}=0, f_{1}:=f \circ$ $\phi$, where $\omega:=\omega(z):=\{\eta \in \mathbf{K}(\alpha): z+\operatorname{Exp}(\eta) \in \Omega\}, \omega_{\epsilon}:=\omega \backslash \log (B(\mathbf{K}(\alpha), z, \epsilon)), z \in \Omega$. Moreover, $C_{n}(\alpha)=C_{1}(\alpha)=\delta$ for each $n \in \mathbb{N}$.

Proof. Using the ${ }_{S} C^{(q, n)}$-diffeomorphism $\phi$, reduce the proof to the case of $f$ on $\Omega$. Consider the differential form $w:=f(\zeta)(\zeta-z)^{-1} d \zeta$ on $\Omega \backslash\{z\}$, then $d w=-(\zeta-z)^{-1}(\partial f /$ $\partial \bar{\zeta}) d \zeta \wedge d \bar{\zeta}$. Let $s \in \mathbb{Z}$ be such that $\inf _{\zeta \in \partial \Omega}|\zeta-z|=|\pi|^{s}$. Take the change of variables 
$\zeta=z+\operatorname{Exp}(\eta)$, hence $(\zeta-z)^{-1} d \zeta=d \eta$; also take $l>s$, then from Corollary 2.3 and Sections 2.2.5 and 2.2.6 it follows that

(i) $\Omega \backslash B\left(\mathbf{K}(\alpha), z,|\pi|^{l}\right) P^{n} d w={ }_{\partial \Omega} P^{n} w-\partial_{c} B\left(\mathbf{K}(\alpha), z,|\pi|^{l}\right) P^{n} w$,

since $f_{1}(z+\operatorname{Exp}(\eta))=: \psi(\eta) \in{ }_{S} C^{(1, n-1)}\left(\omega_{\epsilon}, Y\right)$ and from $\psi \in{ }_{S} C^{(1, n-1)}\left(\omega_{\epsilon}, Y\right)$ it follows that $\psi(x, y) \in{ }_{P, x} C^{(0, n)}\left(\omega_{\epsilon, x}, Y\right)$ and $\psi(x, y) \in{ }_{P, y} C^{(0, n)}\left(\omega_{\epsilon, y}, Y\right)$ for each $\epsilon=\epsilon_{j}$ and for each $x, y$, where $z=(x, y), \omega_{\epsilon, x}=\pi_{x}\left(\omega_{\epsilon}\right), \omega_{\epsilon, y}=\pi_{y}\left(\omega_{\epsilon}\right), \pi_{x}: \mathbf{K} \oplus \alpha \mathbf{K} \rightarrow \mathbf{K}$, and $\pi_{y}: \mathbf{K} \oplus$ $\alpha \mathbf{K} \rightarrow \alpha \mathbf{K}$ are projections, $Y$ is a Banach space over $\mathbf{L}$ such that $\mathbf{K}(\alpha) \subset \mathbf{L}$. The differential form $w$ can be written as $w=f(\zeta) d \log (\zeta-z)$. From $\log (x z)=\log (x)+\log (z)$ for each $x, z \in \mathbb{C}_{\mathbf{p}}^{\times}$it follows that directed going by the oriented loop $\partial_{c} B\left(\mathbf{K}(\alpha), 0,|\pi|^{l}\right)$ changes a branch ${ }_{n} \log$ of $\log$ on 1 in the following manner: ${ }_{n+1} \log (x)-{ }_{n} \log (x)=: \delta \neq 0$ for each $s \in \mathbb{Z}$. In view of Section 2.2.6 there exists $\lim _{l \rightarrow \infty} \partial_{c} B\left(\mathbf{K}(\alpha), z,|\pi|^{l}\right) P^{n} w=: C_{n}(\alpha) f(z)$. Finally ${ }_{\Omega} P^{n}\left((\zeta-z)^{-1} \bar{\partial} f(\zeta) \wedge d \zeta\right)=-{ }_{\Omega} P^{n}\left((\zeta-z)^{-1}(\partial f(\zeta) / \partial \bar{\zeta}) d \zeta \wedge d \bar{\zeta}\right)$, where, for short, we write $f=f(\zeta)=f(\zeta, \bar{\zeta})$.

In view of formulas (2.2), (2.3) and the non-Archimedean Taylor formula for $C^{n_{-}}$ functions (see [22, Theorem 29.4]),

$$
\partial_{c} B\left(\mathbf{K}(\alpha), z,|\pi|^{l)} P^{n}\left[(\zeta-z)^{-1} d \zeta\right]=\partial_{c} B\left(\mathbf{K}(\alpha), z,|\pi|^{l)} P^{1}[d \log (\zeta-z)]+\epsilon\left(\pi^{l}\right)\right.\right.
$$

such that there exists a constant $0<b<\infty$ for which $\left|\epsilon\left(\pi^{l}\right)\right| \leq b|\pi|^{l}$ for each $l \in \mathbb{N}$. On the other hand, due to the Taylor formula for $C^{1}$-functions and formulas (2.2), (2.3),

$$
\partial_{c} B\left(\mathbf{K}(\alpha), z,|\pi|^{l}\right) P^{1}[d \log (\zeta-z)]=\delta+\eta\left(\pi^{l}\right),
$$

where $\lim _{l \rightarrow \infty} \eta\left(\pi^{l}\right)=0$. Therefore, $C_{n}(\alpha)=C_{1}(\alpha)=\delta \neq 0$.

Corollary 2.5. Let suppositions of Theorem 2.4 be satisfied for each $z \in M$ encompassed by $\partial M$, then $\partial f(z) / \partial \bar{z}=0$ for each $z \in M$ encompassed by $\partial M$ if and only if

$$
f(z)=C^{-1} \partial M P^{n}\left\{f(\zeta)(\zeta-z)^{-1} d \zeta\right\}
$$

for each $z \in M$ encompassed by $\partial M$.

Proof. If $\partial f / \partial \bar{\zeta}=0$ on $M$, then the second term in (2.10) is equal to zero, which gives (2.14). Conversely, let (2.14) be satisfied for each $z \in M$ encompassed by $\partial M$. Since $(\partial z / \partial \bar{z})=0, \partial \bar{z} / \partial z=0$, then $\partial\left((\zeta-z)^{-1}\right) / \partial \bar{z}=0$, consequently, $\partial f(z) / \partial \bar{z}=0$.

Corollary 2.6. Let suppositions of Theorem 2.4 be satisfied for each $z \in M$ encompassed by $\partial M$ and $\partial f(z) / \partial \bar{z}=0$ for each $z \in M$ encompassed by $\partial M$, then $f$ is locally $z$-analytic in a neighborhood of each point $\zeta$ in $M$ encompassed by $\partial M$.

Proof. Using the mapping $\phi$ we can consider $\Omega$ instead of $M$. Let $z \in \Omega$ and $B(\mathbf{K} \oplus$ $\alpha \mathbf{K}, z, R) \subset \Omega$ such that $0<R<\inf \{|z-y|: y \in \partial \Omega\}$. Consider $x \in B(\mathbf{K} \oplus \alpha \mathbf{K}, z, R / p)$, then

$$
(\zeta-x)^{-1}=(\zeta-z+z-x)^{-1}=(\zeta-z)^{-1} \sum_{l=0}^{\infty} \frac{(x-z)^{l}}{(\zeta-z)^{l}} \in \mathbf{K}(\alpha),
$$

where $\zeta \in B(\mathbf{K} \oplus \alpha \mathbf{K}, z, R)$. Applying Formula (2.14) we get 
274 Line antiderivations over local fields and their applications

(i)

$$
\begin{aligned}
f(x) & =C^{-1}{ }_{\partial_{c} B} P^{n}\left((\zeta-x)^{-1} f(\zeta) d \zeta\right) \\
& =C^{-1} \sum_{l=0}^{\infty}(x-z)^{l} \partial_{c} P^{n}\left[(\zeta-z)^{-l-1} f(\zeta) d \zeta\right]
\end{aligned}
$$

for each $x \in B(\mathbf{K} \oplus \alpha \mathbf{K}, z, R / p)$, since

(ii)

$$
\left|\partial_{c} B P^{n}\left[(\zeta-z)^{-l-1} f(\zeta) d \zeta\right]\right| \leq\|f\|_{C^{n-1}\left(\partial_{c} B, \mathbf{K}(\alpha)\right)} \max _{j, s=0, \ldots, n-1}\left(\frac{R^{j-l-s}}{|(j+1) !|}\right)
$$

and the series is uniformly converging on $B(\mathbf{K} \oplus \alpha \mathbf{K}, z, R / p)$, where $B=B(\mathbf{K} \oplus \alpha \mathbf{K}, z, R)$, $\mathbf{K} \oplus \alpha \mathbf{K} \subset \mathbf{K}(\alpha)$, hence $f(x)$ is locally $x$-analytic.

Definition 2.7. Let $\Omega$ be as in Section 2.2.3. Two paths $\gamma_{0}: B(\mathbf{K}, 0,1) \rightarrow \Omega$ and $\gamma_{1}: B(\mathbf{K}, 0,1)$ $\rightarrow \Omega$ with common ends $\gamma_{0}(0)=\gamma_{1}(0)=a, \gamma_{0}(\beta)=\gamma_{1}(\beta)=b$ are called affine homotopic in $\Omega$ if there exists a continuous mapping $\gamma(x, y): B(\mathbf{K}, 0,1)^{2} \rightarrow \Omega$ such that

(i) $\gamma(0, y)=\gamma_{0}(y), \gamma(\beta, y)=\gamma_{1}(y)$ for each $y \in B(\mathbf{K}, 0,1)$,

(ii) $\gamma(x, 0)=a, \gamma(x, \beta)=b$ for each $x \in B(\mathbf{K}, 0,1)$,

(iii) there exists a sequence $\left\{\gamma_{n}(x, y): n \in \mathbb{N}\right\}$ of continuous mappings, $\gamma_{n}: B(\mathbf{K}, 0,1)^{2}$ $\rightarrow \Omega$, such that each $\gamma_{n}$ is locally affine and $\left\{\gamma_{n}: n\right\}$ converges uniformly to $\gamma$ on $B(\mathbf{K}, 0,1)^{2}$, where $\gamma_{n}(x, y)=(1-x / \beta) \gamma_{n}(0, y)+x \gamma_{n}(\beta, y) / \beta$ for each $x \in B(\mathbf{K}, 0,1)$, $\gamma_{n}(0, y)$ and $\gamma_{n}(\beta, y)$ are locally affine (see Section 2.2.4). In particular, for $a=b$ this produces the definition of affine homotopic loops. $\Omega$ is called (or $M$ ) affine homotopic to a point if $\partial \Omega$ (resp., $\partial M$ ) is affine homotopic to a point $z$ in $\Omega$ (resp., $z$ in $M$; see Section 2.2.5).

Theorem 2.8. Let conditions of Theorem 2.4 be satisfied for each $z \in M$ and let $M$ be affine homotopic to a point, where $\partial f(z, \bar{z}) / \partial \bar{z}=0$ for each $z \in M$ encompassed by $\partial M$. Then

$$
{ }_{\gamma_{0}} P^{n}[f d \zeta]={ }_{\gamma_{1}} P^{n}[f d \zeta]
$$

for each two paths $\gamma_{0}$ and $\gamma_{1}$ which are affine homotopic in $M$.

Proof. Using the diffeomorphism $\phi$ we can consider $\Omega$ instead of $M$. For each $\epsilon>0$ there exists a finite partition of a suitable subset $\Omega_{\epsilon}$ into finite union of parallelepipeds of diameter less than $\epsilon$ in the proof of Theorem 2.2, where $\Omega_{\epsilon} \subset\{z \in \Omega: d(z, \partial \Omega)<\epsilon\}$, $\operatorname{cl}\left(\bigcup_{\epsilon>0} \Omega_{\epsilon}\right)=\Omega$. In view of Corollary $2.5,0=\left.f(z)(z-\zeta)\right|_{z=\zeta}=C(\alpha)^{-1} \partial \xi P^{n}[f(\zeta) d \zeta]$ for each such parallelepiped $\xi$. Therefore, there exists a sequence $\left\{\gamma_{l}: l\right\}$ of affine homotopy such that $\gamma_{l}(0, y)$ and $\gamma_{l}(\beta, y)$ are contained in the union $\bigcup_{\xi \subset \Omega} \partial \xi$ for each $\epsilon_{l}=|\pi|^{l}$, $l \in \mathbb{N}$. Since ${ }_{\gamma_{l}(0, *)} P^{n}[f d \zeta]=\gamma_{l}(\beta, *) P^{n}[f d \zeta]$ for each $l$ and taking $l$ tending to infinity we get (2.18) due to continuity of the operator $P^{n}$. 
Corollary 2.9. Let $f$ satisfy conditions of Corollary 2.6 with $\Omega=B(\mathbf{K} \oplus \alpha \mathbf{K}, z, R)$. Then

(i)

$$
\begin{aligned}
|f(x)| & \leq|C|^{-1} \max _{j, s=0, \ldots, n-1}\left(\left\|f^{(j-s)}\right\|_{C^{0}\left(\partial_{c} B, Y\right)} s !\left(\begin{array}{l}
j \\
s
\end{array}\right) \frac{R^{j-s-1}}{(j+1) !}\right) \\
& \leq|C|^{-1}\|f\|_{C^{(n-1)}\left(\partial_{c} B, Y\right)} \max _{j, s=0, \ldots, n-1}\left(\frac{R^{j-s-1}}{(j+1) !}\right) .
\end{aligned}
$$

Proof. From

$$
\partial_{\zeta}^{j} f(\zeta)(\zeta-x)^{-1}=\sum_{s=0}^{j} s !\left(\begin{array}{l}
j \\
s
\end{array}\right)(-1)^{s} f^{(j-s)}(\zeta)(\zeta-x)^{-1-s}
$$

$\left|\zeta_{l+1}-\zeta_{l}\right| \leq R$ on $\partial_{c} B$, and Section 2.1, inequality (i) follows.

Remark 2.10. The field $\mathbf{K}$ is locally compact, then $\mathbf{T}_{q}$ is not contained in $\mathbf{K}$, where $\mathbf{T}_{q}$ is a group of all $q^{n}$-roots $b$ of the unity: $b^{l}=1, l=q^{n}, n \in \mathbb{N}, q$ is the prime number, since $\operatorname{dim}_{\mathbb{Q}_{\mathbf{p}}} \mathbb{Q}_{\mathbf{p} \mathbf{T}_{q}}=\infty$ for $\mathbb{Q}_{\mathbf{p}} \subset \mathbf{K}$, and $\mathbf{K}$ would be nonlocally compact whenever $\mathbf{T}_{q} \subset \mathbf{K}$, which is impossible by the supposition on $\mathbf{K}$. Therefore, there exists $\min \left\{s \in \mathbb{N}: b^{q} \in\right.$ $\mathbf{K}, b \notin \mathbf{K}$, where $b \neq 1$ is the $q^{s+1}$-root of the unity $\}$. Hence there exists $\zeta \in \mathbf{K}$ such that $\zeta^{1 / q} \notin \mathbf{K}$. In particular, it is true for $q=2$. Therefore, each local field $\mathbf{K}$ has a quadratic extension $\mathbf{K}(\alpha)$ such that $\alpha \notin \mathbf{K}$. In the particular case $\mathbf{K}=\mathbb{Q}_{\mathbf{p}}$ there exists the finite field $\mathbf{F}_{\mathbf{p}}:=R / P$ (see Section 2.2.1). Then $\mathbf{F}_{\mathbf{p}} \backslash\{0\}$ is the multiplicative group consisting of $p-1$ elements. If $p=4 n+1$, where $1 \leq n \in \mathbb{N}$, then $\mathbb{Q}_{\mathbf{p}}$ contains $i=(-1)^{1 / 2}$.

LEMma 2.11. If $f$ is locally $z$-analytic on $M$, where $M$ is a locally compact $C^{(0, n)}$-manifold satisfying conditions of Sections 2.2.5 and 2.2.6, $\phi^{-1}(M)=\Omega \subset \mathbf{K}(\alpha), \operatorname{dim}_{\mathbf{K}} M=2,2 \leq r \in$ $\mathbb{N}$, then $\partial f(z, \bar{z}) / \partial \bar{z}=0$ on $M$.

Proof. Using the diffeomorphism $\phi$ we can consider $\Omega$ instead of $M$. Since for each $z \in \Omega$ there exists $0<R<\infty$ such that $B:=B(\mathbf{K} \oplus \alpha \mathbf{K}, z, R) \subset \Omega$ and $f(z, \bar{z})=\sum_{k=0}^{\infty}(\zeta-z)^{k} f_{k}$ on $B$, where $f_{k} \in Y$, then there exist $\partial f / \partial z$ and $\partial f / \partial \bar{z}=0$ on $B$. Since $z \in \Omega$ is arbitrary and such balls form the covering of $\Omega$, then $\partial f / \partial \bar{z}=0$ on $\Omega$.

Remark 2.12. Let $n \geq 1$, then $(d / d z)_{\Omega} P^{n}=I: C^{n-1}(\Omega, \mathbf{L}) \rightarrow C^{n-1}(\Omega, \mathbf{L})$. But $P^{n} d / d z \neq I$ on $C^{n}(\Omega, \mathbf{L})$, where $P^{n} d / d z: C^{n}(\Omega, \mathbf{L}) \rightarrow C^{n}(\Omega, \mathbf{L})$. If ${ }_{P} C^{n}(\Omega, \mathbf{L})$ is dense in $C^{n}(\Omega, \mathbf{L})$, then $P^{n} d / d z$ will have the continuous extension $I$ on $C^{n}(\Omega, \mathbf{L})$, since $P^{n} d / d z$ is the continuous operator from $C^{n}$ into $C^{n}$ and $\left.P^{n}(d / d z)\right|_{P} C_{0}^{n}=I$. Therefore, ${ }_{P} C^{n}(\Omega, \mathbf{L})$ is not dense in $C^{n}(\Omega, \mathbf{L})$. On the other hand, $C^{1}(\Omega, \mathbf{L})={ }_{P} C_{0}^{1}(\Omega, \mathbf{L}) \oplus N^{1}$, where $N^{1}:=\left\{f \in C^{1}: f^{\prime}=0\right\}$ is the closed $\mathbf{L}$-linear subspace in $C^{1}$ (see [21, Theorem 5.1, Corollary 5.5]).

Theorem 2.13. Let $f$ be a function on $M$ over $\mathbf{K}$ satisfying the conditions in Sections 2.2.5 and 2.2.6, let $\gamma$ be a loop in $M$ satisfying the conditions in Section 2.2.4, and let $\gamma$ be affine homotopic to a point in $M$, $\operatorname{dim}_{\mathbf{K}} M=2, \phi^{-1}(M)=\Omega \subset \mathbf{K}(\alpha), 2 \leq r \in \mathbb{N}, f$ satisfying condition (2.11) for each $z \in M$, and $\partial f(z, \bar{z}) / \partial \bar{z}=0$ on $M$, then ${ }_{\gamma} P^{n} f=0$. 
Proof. Let $V$ be a submanifold in $M$ such that $\partial V=\gamma$. In view of Theorem $2.8{ }_{\gamma} P^{n}[f d \zeta]=$ ${ }_{\epsilon} P^{n}[f d \zeta]$, where $\gamma$ and $\gamma_{\epsilon}$ are affine homotopic and $0<\operatorname{diam}\left(\gamma_{\epsilon}\right)<\epsilon$. In view of continuity of the operator $P^{n}$ there exists $\lim _{\epsilon \rightarrow 0} \gamma_{\epsilon} P^{n}[f d \zeta]=0$.

Theorem 2.14. If $f$ satisfies condition (2.11), a manifold $M$ over $\mathbf{K}$ satisfies the conditions in Sections 2.2.5 and 2.2.6, and $M$ is affine homotopic to a point, $\operatorname{dim}_{\mathrm{K}} M=2,2 \leq$ $r \in \mathbb{N}$, and ${ }_{\gamma} P^{n} f=0$ for each loop $\gamma$ in $M$ satisfying the conditions in Section 2.2.4, then $\partial f(z, \bar{z}) / \partial \bar{z}=0$ for each $z \in M$ encompassed by $\partial M$.

Proof. Using the diffeomorphism $\phi$ we can consider $\Omega$ instead of $M$. Choose a marked point $z_{0}$ in $M$. Let $\eta$ be a path joining points $z_{0}$ and $z$ and satisfying the conditions in Section 2.2.4. From ${ }_{\gamma} P^{n} f=0$ it follows that ${ }_{\eta} P^{n} f$ does not depend on $\eta$ besides points $z_{0}=\eta(0)$ and $z=\eta(\beta)$, since each two points in $\Omega$ can be joined by an affine path, hence it is possible to put $F(z):={ }_{\eta ; \eta}(0)=z_{0} ; \eta(\beta)=z P^{n} f$ such that $F$ is a function on $\Omega$. In view of formulas in Section 2.2.6(i)-(iv),

(i) $\partial F(z) / \partial z=f(z)$.

In view of Theorem 2.4,

(ii) $0={ }_{\gamma} P^{n}(f(\zeta) d \zeta)=-C^{-1}{ }_{U}^{n}((\partial f(\zeta, \bar{\zeta}) / \partial \bar{\zeta}) d \zeta \wedge d \bar{\zeta})$

for each submanifold $V$ in $M$ with the loop $\gamma=\partial V, \operatorname{dim}_{K} V=2$. Since $V$ is arbitrary, then $\bar{\partial} f(z, \bar{z})=0$ at each point $z \in M$ encompassed by $\partial M$.

COROLlary 2.15. Let conditions of Theorem 2.14 be satisfied, then $f$ has an antiderivative $F$ such that $F^{\prime}=f$ on $M$.

Lemma 2.16. Let $\Omega$ be a clopen compact subset in $\mathbf{K}^{m}$, then for each $y \in \Omega$ there exists a ball $B$ such that $y \in B \subset \Omega,\left.{ }_{P} C^{\xi}(\Omega, Y)\right|_{B}={ }_{P} C^{\xi}(B, Y)$, and $\left.{ }_{S} C^{\xi}(\Omega, Y)\right|_{B}={ }_{S} C^{\xi}(B, Y)$ for each $\xi$, where $\left.{ }_{P} C^{\xi}(\Omega, Y)\right|_{B}:=\left\{\left.g\right|_{B}: g \in{ }_{P} C^{\xi}(\Omega, Y)\right\}$ and $\left.{ }_{S} C^{\xi}(\Omega, Y)\right|_{B}:=\left\{\left.g\right|_{B}: g \in{ }_{S} C^{\xi}(\Omega, Y)\right\}, Y$ is a Banach space over $\mathbf{L}, \xi=(t, n), 0 \leq n \in \mathbb{Z}, 0 \leq t \in \mathbb{Z}, 1 \leq n$ for ${ }_{P} C^{\xi}, 1 \leq t$ for ${ }_{S} C^{\xi}$.

Proof. Let $\sigma$ be an approximation of the unity in $U$. In view of Section 2.1, it is sufficient to consider the case $m=1$. Choose $R=\rho^{s+1 / 2}$ for sufficiently large $s \in \mathbb{N}$ such that $0 \in$ $B:=B(\mathbf{K}, 0, R)$. If $x \in B(\mathbf{K}, y, R)$, then $\sigma_{s}(x)=\sigma_{s}(y)$ due to formula (iii) in Section 2.1. From formula (ii) in Section 2.1, it follows that $\sigma_{l}(x)=\sigma_{l}(y)$ for each $l<s$. Moreover, $\sigma_{l}(x)=: x_{l} \in B$ for each $l \geq s$, since $\rho^{s+1}<R<\rho^{s}$ and the valuation group $\Gamma_{\mathrm{K}}:=\left\{|q|_{\mathrm{K}}: 0 \neq\right.$ $q \in \mathbf{K}\}$ of $\mathbf{K}$ is discrete, since $\mathbf{K}$ is locally compact. Therefore,

(i) $\left.\left[{ }_{U} P^{n} f(x)\right]-{ }_{B} P^{n} f(x)\right]=\sum_{j=0}^{n-1} \sum_{k=0}^{s-1} f^{(j)}\left(x_{k}\right)\left(x_{k+1}-x_{k}\right)^{j+1} /[(j+1) !]$

for each $f \in C^{\xi}(U, Y)$, where $x_{k}=y_{k}$ is fixed, and the term on the right-hand side of (i) is independent of $x \in B$, that is, constant on $B$. Hence $g \in{ }_{P} C^{\xi}(U, Y)$ if and only if $\left.g\right|_{B} \in$ ${ }_{P} C^{\xi}(B, Y)$. From (2.3) and $\chi_{\Omega} \chi_{B}=\chi_{B}=\left.\chi_{\Omega}\right|_{B}$, the statement of this lemma follows.

Definition 2.17. Let a manifold $M$ satisfy Theorem 2.4, $f \in C^{(q, n-1)}(M, Y), 0 \leq q \in \mathbb{Z}$, $1 \leq n \in \mathbb{N}, Y$ is a Banach space over $\mathbf{L}, \mathbf{K}(\alpha) \subset \mathbf{L}$. Then put in the sense of distributions

$$
{ }_{M} P^{n}\left(g f^{\prime}\right):=-{ }_{M} P^{n}\left(g^{\prime} f\right)
$$


for each $g \in{ }_{S} C^{(1, n-1)}\left(M, Y^{*}\right)$ with

$$
\operatorname{supp}(g) \subset \widetilde{M}:=\{z \in M: z \text { is encompassed by } \partial M\}
$$

where $M \hookrightarrow \mathbf{K}(\alpha)^{N}$ (see Theorem 2.1), $Y^{*}$ is the topologically dual space of all $\mathbf{L}$-linear continuous functionals $\theta: Y \rightarrow \mathbf{L}$, the valuation group $\Gamma_{\mathbf{L}}$ of $\mathbf{L}$ is discrete.

THeOREM 2.18. Let a manifold $M$ satisfy Theorem 2.4 and let $f$ satisfy Theorem 2.4 for each $z \in M$, then the function

(1) $u(z):=\bar{z} C_{n}(\alpha)^{-1}{ }_{\partial M} P^{n}\left[f(\zeta)(\zeta-z)^{-1} d \zeta\right]-C_{n}(\alpha)^{-1}{ }_{M} P^{n}\left[f(\zeta)(\zeta-z)^{-1} d \bar{\zeta} \wedge d \zeta\right]$

is a solution of the equation

(2) $\partial u(z) / \partial \bar{z}=f(z)$

in the sense of distributions for each $z \in M$ encompassed by $\partial M$.

Proof. The space ${ }_{P} C^{(q, n)}(M, Y)$ is dense in $C^{(q, n-1)}(M, Y)$. Indeed, for each $\delta>0$ and for each continuous function $f \circ \phi$ on $\Omega$ or a continuous partial difference quotient $w_{q}:=\bar{\Phi}^{q} f \circ \phi\left(x ; h_{1}^{\otimes s_{1}}, \ldots, h_{m}^{\otimes s_{m}} ; \zeta_{1}, \ldots, \zeta_{q}\right)$ on a domain containing $\Omega^{q+1} \times B(\mathbf{K}, 0,1)^{t}$ with $0 \leq t \leq(n-1) m, 0 \leq s_{j} \leq n$, for each $j=1, \ldots, m, t=s_{1}+\cdots+s_{m}, x, x+\zeta_{j} h_{j} \in \Omega, h_{j} \in$ $V, \zeta_{j} \in B(\mathbf{K}, 0,1), V$ is a neighbourhood of 0 in $\mathbf{K}^{m}, \Omega+V \subset \Omega$ (see $\left.[15,16]\right), m:=$ $\operatorname{dim}_{\mathbf{K}} M$, there exists a finite partition of $\Omega^{q+1}$ into a disjoint union of balls $B_{j}$ such that on each $B_{j}$ the variation $\operatorname{var}\left(w_{q}\right):=\sup _{x, y \in B_{j}}\left|w_{q}(x)-w_{q}(y)\right|<\delta$, since $M$ is compact and for each covering of $M$ by such balls there exists a finite subcovering. Therefore, in $C^{(q, n-1)}(\Omega, Y)$ the subspace $\Sigma^{(q, n-1)}(\Omega, Y)$ of all $C^{(q, n-1)}(\Omega, Y)$-functions $f$ such that $w_{(n-1) m}$ corresponding to $f$ is locally constant on the diagonal $\Delta \Omega^{(n-1) m+1}:=\left\{\left(y_{1}, \ldots\right.\right.$, $\left.\left.y_{(n-1) m+1}\right) \in \Omega^{(n-1) m+1}: y_{1}=\cdots=y_{(n-1) m+1}\right\}$ is dense. Since the operator ${ }_{\Omega} P^{n}$ is continuous, then ${ }_{\Omega} P^{n}\left(\Sigma^{(q, n-1)}(\Omega, Y)\right)$ is dense in $C_{0}^{(q, n-1)}(\Omega, Y)$ and ${ }_{S} \Sigma^{(q+1, n-1)}(M, Y):=\{f \in$ $\left.{ }_{s} C^{(q+1, n-1)}(M, Y): f=y_{l}+{ }_{M} P_{x_{l}}^{n} g_{l} \forall l=1, \ldots, m, y_{l} \in Y, g_{l} \in \Sigma^{(q, n-1)}(M, Y)\right\}$ is dense in ${ }_{S} C^{(q+1, n-1)}(M, Y)$. From ${ }_{S} \sum^{(q+1, n-1)}(M, Y) \subset{ }_{S} C^{(q+1, n-1)}(M, Y) \subset{ }_{P} C^{(q, n)}(M, Y) \subset$ $C^{(q, n-1)}(M, Y)$ it follows that ${ }_{S} C^{(q+1, n-1)}(M, Y)$ is dense in $C^{(q, n-1)}(M, Y)$. In particular, take $\mathbf{L}$ such that $\mathbf{K}(\alpha) \subset \mathbf{L}$. Since ${ }_{P} C^{(0, n)}\left(M, Y^{*}\right) \subset\left\{g^{\prime}: g \in{ }_{S} C^{(1, n-1)}\left(M, Y^{*}\right)\right\} \subset$ $C^{(0, n-1)}\left(M, Y^{*}\right)$, the family of functionals $\left\{{ }_{M} P^{n}\left(g^{\prime} f\right): g \in{ }_{S} C^{(1, n-1)}\left(M, Y^{*}\right)\right\}$ separates points of $C^{(q, n-1)}(M, Y)$, since $Y^{*}$ separates points of $Y$ for discrete $\Gamma_{\mathbf{L}}$ (see [28, Theorem 4.15]). In view of (2.21), it is sufficient to prove this theorem for $f \circ \phi(z+\operatorname{Exp}(\eta))=$ : $\psi(\eta) \in{ }_{P} C^{(0, n)}\left(\omega_{\epsilon, x}, Y\right) \cap{ }_{P} C^{(0, n)}\left(\omega_{\epsilon, y}, Y\right)$ for each $\epsilon=\epsilon_{j}$, where $\omega(z):=\omega:=\{\eta \in \mathbf{K}(\alpha)$ : $z+\operatorname{Exp}(\eta) \in \Omega\}, z \in \Omega, \omega_{\epsilon}=\omega \backslash \log (B(\mathbf{K}(\alpha), z, \epsilon)), \epsilon=\epsilon_{j}, \omega_{\epsilon, x}=\pi_{x}\left(\omega_{\epsilon}\right), \omega_{\epsilon, y}:=\pi_{y}\left(\omega_{\epsilon}\right)$.

Using the diffeomorphism $\phi$ we can consider $\Omega$ instead of $M$. Choose a clopen ball $B:=B\left(\mathbf{K} \oplus \mathbf{K}(\alpha), z_{0}, R\right) \subset \Omega$ containing a point $z_{0} \in \Omega$ and its characteristic function $\chi:=\chi_{B}$. Then $(f \chi)_{1} \in{ }_{P} C^{(0, n)}\left(b_{x}, Y\right) \cap{ }_{P} C^{(0, n)}\left(b_{y}, Y\right)$ for suitable $0<R<\infty$, where $b:=$ $\left\{\eta \in \mathbf{K}(\alpha): z_{0}+\operatorname{Exp}(\eta) \in B\right\}, b_{x}:=\pi_{x}(b), b_{y}:=\pi_{y}(b)$ (see Lemma 2.16). Using the affine mapping $z \mapsto\left(z-z_{0}\right)$ we can consider 0 instead of $z_{0}$. Then $B$ is the additive group. We can take $R>0$ sufficiently small such that each point of $B$ is encompassed by $\partial \Omega$. Therefore,

(3) $u=u_{1}+u_{2}$, where

(4) $u_{1}(z):=C^{-1} \bar{z}_{\partial \Omega} P^{n}\left[f(\zeta)(\zeta-z)^{-1} d \zeta\right]-C^{-1}{ }_{\Omega} P^{n}\left[\chi(\zeta) f(\zeta)(\zeta-z)^{-1} d \bar{\zeta} \wedge d \zeta\right]$,

(5) $u_{2}(z):=-C_{\Omega}^{-1} P^{n}\left[(1-\chi(\zeta)) f(\zeta)(\zeta-z)^{-1} d \bar{\zeta} \wedge d \zeta\right]$. 
278 Line antiderivations over local fields and their applications

From (5) it follows that $\partial u_{2}(z) / \partial \bar{z}=0$ on $B$. From (4) it follows that

$$
\begin{aligned}
u_{1}(z)= & C^{-1} \bar{z}_{\partial \Omega} P^{n}\left[f(\zeta)(\zeta-z)^{-1} d \zeta\right] \\
& -C^{-1}{ }_{\Omega} P^{n}\left[\chi(\zeta+z) f(\zeta+z) \zeta^{-1} d(\bar{\zeta}+\bar{z}) \wedge d(\zeta+z)\right]
\end{aligned}
$$

for each $z \in B$, since $B+B=B \subset \Omega$. Since $\partial_{c} B$ encompasses $z$ and

$$
{ }_{\partial_{c} B} P^{n}\left[f(\zeta)(\zeta-z)^{-1} d \zeta\right]=\sum_{l=0}^{\infty}(z-y)^{l} \partial_{c} B P^{n}\left[f(\zeta)(\zeta-y)^{-l-1} d \zeta\right]
$$

for each $z \in B$ with $|z-y|<R$ due to formula (ii) in the proof of Corollary 2.6 for this antiderivative, then

(6) $\partial\left\{\partial_{c} B P^{n}\left[f(\zeta)(\zeta-z)^{-1} d \zeta\right]\right\} / \partial \bar{z}=0$. In view of formulas (2.1)-(2.4) and Section 2.2.5,

$$
\begin{aligned}
\frac{\partial u_{1}}{\partial \bar{z}}(z)= & C^{-1}{ }_{\partial \Omega} P^{n}\left[f(\zeta)(\zeta-z)^{-1} d \zeta\right] \\
& -C^{-1}{ }_{\Omega} P^{n}\left\{\left[\bar{\partial}_{\zeta+z}[\chi(\zeta+z) f(\zeta+z)] \zeta^{-1} \wedge d(\zeta+z)\right]\right\}
\end{aligned}
$$

consequently,

$$
\begin{aligned}
\frac{\partial u_{1}}{\partial \bar{z}}(z)= & C^{-1}{ }_{\partial \Omega} P^{n}\left[f(\zeta)(\zeta-z)^{-1} d \zeta\right] \\
& -C^{-1}{ }_{\Omega} P^{n}\left\{\left[\bar{\partial}_{\zeta}(\chi(\zeta) f(\zeta)) \wedge d \zeta\right](\zeta-z)^{-1}\right\}
\end{aligned}
$$

In view of Theorem 2.4 we get the statement of this theorem, since $B$ and $y$ are arbitrary forming covering of each point $z \in \Omega$ encompassed by $\partial \Omega$.

Definition 2.19. Let $M$ be a manifold over K satisfying Theorem 2.4. If $f \in C^{(q, n-1)}(M, Y)$ and for each loop $\gamma$ in $M,{ }_{\gamma} P^{n} f=0$, then $f$ is called $(q, n)$-antiderivationally holomorphic on $M$, where $Y$ is a Banach space over $\mathbf{L}, \mathbf{K}(\alpha) \subset \mathbf{L}$. If $f \in C^{(q, n)}(M, Y)$ and $\bar{\partial} f(z)=0$ for each $z \in M$, then $f$ is called derivationally $(q, n)$-holomorphic.

Theorem 2.20. Let $\Omega$ be a clopen compact subset in $(\mathbf{K} \oplus \alpha \mathbf{K})^{m}$. Consider the following conditions.

(i) $f$ satisfies (2.11) and $\bar{\partial} f(z)=0$ for each $z \in \Omega$ with $z_{j}$ encompassed by $\partial \Omega_{j}$ for each $j=1, \ldots, m$, where $\Omega_{j}=\pi_{j}(\Omega), \pi_{j}(\zeta)=\zeta_{j}$ for each $\zeta=\left(\zeta_{1}, \ldots, \zeta_{m}\right), \zeta_{j} \in \mathbf{K} \oplus \alpha \mathbf{K}$. 
(ii) $f$ is $(0, n)$-antiderivationally holomorphic on

$$
\widetilde{\Omega}:=\left\{z \in \Omega: z_{j} \text { is encompassed by } \partial \Omega_{j} \forall j=1, \ldots, m\right\} .
$$

(iii) $f \in C^{(0, n-1)}(\Omega, Y)$ and for each polydisc $B=B_{1} \times \cdots \times B_{m} \subset \Omega, B_{j}=B\left(\mathbf{K}(\alpha), z_{0, j}\right.$, $R_{j}$ ) for each $j=1, \ldots, m, f(z)$ is given by the antiderivative

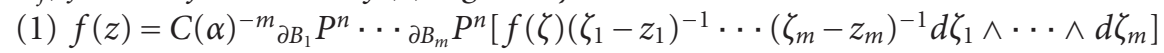
for each $z \in B$ with $z_{j}$ encompassed by $\partial B_{j}$ for each $j$.

(iv) $f$ is locally $z$-analytic, that is,

(2) $f(z)=\sum_{k} a_{k}(z-\zeta)^{k}$ in some neighborhood of $\zeta \in \widetilde{\Omega}, a_{k} \in Y, k=\left(k_{1}, \ldots, k_{m}\right)$, $0 \leq k_{j} \in \mathbb{Z}, z^{k}:=z_{1}^{k_{1}} \cdots z_{m}^{k_{m}}, z=\left(z_{1}, \ldots, z_{m}\right), z_{j} \in \mathbf{K}(\alpha)$.

(v) $f \in C^{\infty}(\Omega, Y)$.

(vi) $f \in C^{(0, n-1)}(\Omega, Y)$ and for every polydisc $B$ as in (iii) and each multiorder $k$ as in (iv) derivatives are given by

(3) $\partial_{z}^{k} f(z)=k ! C(\alpha)^{-m} \partial_{1} P^{n} \cdots \partial_{m} P^{n}\left[f(\zeta)\left(\zeta_{1}-z_{1}\right)^{-k_{1}-1} \cdots\left(\zeta_{m}-z_{m}\right)^{-k_{m}-1} d \zeta_{1}\right.$ $\left.\wedge \cdots \wedge d \zeta_{m}\right]$.

(vii) The coefficients in formula (2) are determined by the equation

(4) $a_{k}=\partial_{z}^{k} f(z) / k !$

(viii) The power series (2) converges uniformly in each polydisk $B \subset \widetilde{\Omega}$ with sufficiently small $b:=\max \left(R_{1}, \ldots, R_{m}, 1\right)$.

Then from (i) properties (ii), (iii), (iv), (v), (vi), (vii), and (viii) follow. Properties (iii) and (vi) are equivalent. From (iii) properties (iv), (v), (vii), and (viii) follow. In the subspace

$$
\begin{aligned}
\{f \in & C^{(0, n-1)}(\Omega, Y): f\left(z_{1}, \ldots, z_{l-1}, z_{l}+\operatorname{Exp}(\eta), z_{l+1}, \ldots, z_{m}\right) \\
& \left.=: \psi_{l}(\eta) \in{ }_{s} C^{(1, n-1)}\left(\omega_{l, \epsilon}, Y\right) \text { for each } l=1, \ldots, m \text { and each } \epsilon=\epsilon_{j}\right\}
\end{aligned}
$$

where $\omega_{l}:=\omega_{l}(z):=\left\{\eta \in \mathbf{K}(\alpha):\left(z_{1}, \ldots, z_{l-1}, z_{l}+\operatorname{Exp}(\eta), z_{l+1}, \ldots, z_{m}\right) \in \Omega\right\}, \omega_{l, \epsilon}:=\omega_{l} \backslash$ $\log \left(B\left(\mathbf{K}(\alpha), z_{l}, \epsilon\right)\right), z \in \Omega, Y$ is a Banach space over $\mathbf{L}$ such that $\mathbf{K}(\alpha) \subset \mathbf{L}$, properties (i), (ii), (iii), and (iv) are equivalent.

Proof. From (i), (iv) follows due to repeated application of Corollary 2.6. From (i), (iii) follows due to repeated application of Corollary 2.5. Other statements follow from Theorems 2.13 and 2.14, Lemma 2.11, and formulas (2.16) and (2.17), since from formula (3) it follows that

(5) $\left|\partial_{z}^{k} f(z) / k !\right| \leq|C(\alpha)|^{-m} \sup _{\zeta \in \Omega, l}\left|\partial_{z}^{l} f(\zeta)\right| \max _{l}\left[b^{|l|-|k|} /(l+\bar{e}) !\right]<\infty$,

where $l=\left(l_{1}, \ldots, l_{m}\right), 0 \leq l_{j} \in \mathbb{Z}, l_{j}<n$ for each $j=1, \ldots, m,|l|=l_{1}+\cdots+l_{m}, \bar{e}:=$ $(1, \ldots, 1) \in \mathbb{Z}^{m}, b:=\max \left(R_{1}, \ldots, R_{m}\right)$. The series (2) with $a_{k}$ given by (4) converges uniformly in $B$, when $\overline{\lim }_{k}\left|a_{k}\right|^{1 /|k|} b<1$.

Corollary 2.21. Spaces $C^{l a}(\Omega, \mathbf{K}(\alpha))$ of locally analytic functions $f: \Omega \rightarrow \mathbf{K}(\alpha)$ and the space $C^{(q, n), \text { dh }}(\Omega, \mathbf{K}(\alpha))$ of all derivationally $(q, n)$-holomorphic functions are rings. If $f$ is derivationally $(q, n)$-holomorphic and $f \neq 0$ on $\Omega$, then $1 / f$ is derivationally $(q, n)$ holomorphic on $\Omega$. 
Corollary 2.22. If $f$ satisfies (2.11) and there exists $\zeta \in \Omega$ encompassed by $\partial \Omega$ such that $\partial_{z}^{k} f(\zeta)=0$ for each $k$, then there exists a polydisc $B \subset \widetilde{\Omega}$ (see Theorem 2.20) such that $f=0$ on $B$.

Proof. In view of Theorem 2.20 there exists a polydisc $B$ where Theorem 2.20(iv)(2) and (vii)(4) are accomplished.

Remark 2.23. $\left.{ }_{U} P^{n} z^{k}\right|_{a} ^{b} \neq\left(b^{k+1}-a^{k+1}\right) /(k+1)$ for each $a \neq b \in U$, where $k>0$. In view of [22, Corollary 54.2, Theorem 54.4] and [1] the spaces $C^{l a}(\Omega, \mathbf{K}(\alpha)) \cap C^{((q, n), d h)}(\Omega, \mathbf{K}(\alpha))$, $C^{l a}(\Omega, \mathbf{K}(\alpha)) \cap{ }_{P} C^{(q, n)}(\Omega, \mathbf{K}(\alpha))$,

$$
\left\{f \in C^{l a}(\Omega, \mathbf{K}(\alpha)): f \text { is }(q, n) \text {-antiderivationally holomorphic }\right\}
$$

are infinite-dimensional over $\mathbf{K}(\alpha)$, since the condition of the local analyticity means that the expansion coefficients $a(m, f)$ of the function $f$ in the Amice polynomial basis $\bar{Q}_{m}$ are such that $\lim _{|m| \rightarrow \infty} a(m, f) / P_{m}(\tilde{u}(m))=0$, where $P_{m}$ are definite polynomials (see [17, formulas 2.6(i)-(iii)]).

Theorem 2.24. Let $\Omega$ and $f$ be as in Theorem 2.20(i). If $\zeta$ is zero of $f$ such that $f$ does not coincide with 0 on each neighborhood of $\zeta$, then there exists $n \in \mathbb{N}$ such that

$$
f(z)=(z-\zeta)^{n} g(z)
$$

where $g$ is analytic and $\phi \neq 0$ on some neighborhood of $z$.

Proof. In view of Theorem 2.20 there exists a neighborhood $V$ of $\zeta$ such that $f$ has a decomposition into converging series Theorem 2.20(iv)(2). If $a_{k}=0$ for each $k$, then $\left.f\right|_{V}=0$. Therefore, there exists a minimal $k$ denoted by $l$ such that

$$
f(z)=\sum_{k=l}^{\infty} a_{k}(z-\zeta)^{k},
$$

putting

$$
g(z)=\sum_{k=0}^{\infty} a_{k+l}(z-\zeta)^{k} .
$$

Since $a_{l} \neq 0$, there exists a neighborhood $\zeta \in W \subset V$ such that $\left.g\right|_{W} \neq 0$.

Theorem 2.25. Let $\Omega$ and two functions $f_{1}$ and $f_{2}$ satisfy Theorem 2.20(i) such that $f_{1}(z)$ $=f_{2}(z)$ for each $z \in E$, where $E \subset \Omega$ and $E$ contains a limit point $\zeta \in E^{\prime}$. Then there exists a clopen subset $W$ in $\Omega$ such that $\zeta \in W$ and $\left.f_{1}\right|_{W}=\left.f_{2}\right|_{W}$.

Proof. Put $f:=f_{1}-f_{2}$, then $f$ satisfies Theorem 2.20(i) and $f(\zeta)=0$. In view of Theorem $\left.2.24 f\right|_{W}=0$ for some clopen $W$ in $\Omega$, where $\zeta \in W$.

Theorem 2.26. Let $f$ satisfy (2.11) and let it be derivationally $(0, n)$-holomorphic on $\Omega:=$ $\left\{z \in(\mathbf{K} \oplus \alpha \mathbf{K})^{m}: R_{1} \leq|z-\xi| \leq R_{2}\right\}$, where $0<R_{1}<R_{2}<\infty, R_{1}$ and $R_{2} \in \Gamma_{\mathbf{K}}$. Then

$$
f(z)=\sum_{k} a_{k}(z-\xi)^{k}
$$


for each $z \in \Omega$ with $R_{2}>|z|>R_{1}$, where

$$
a_{k}=C(\alpha)^{-m}{ }_{\partial_{c} B_{R, 1}} P^{n} \cdots \partial_{c} B_{R, m} P^{n}\left[\left(\zeta_{1}-\xi_{1}\right)^{-k_{1}-1} \cdots\left(\zeta_{m}-\xi_{m}\right)^{-k_{m}-1} f(\zeta) d \zeta_{1} \wedge \cdots \wedge d \zeta_{m}\right]
$$

for each $k \in \mathbb{Z}^{m}, R_{1}<R<R_{2}, B_{R, l}:=\left\{z_{l} \in \mathbf{K} \oplus \alpha \mathbf{K}:\left|z_{l}-\xi_{l}\right| \leq R\right\}, k=\left(k_{1}, \ldots, k_{m}\right), k_{l} \in \mathbb{Z}$, $l=1, \ldots, m$.

Proof. Let $\pi_{l}(z)=z_{l}$ for each $z=\left(z_{1}, \ldots, z_{m}\right) \in(\mathbf{K} \oplus \alpha \mathbf{K})^{m}$, where $z_{l} \in \mathbf{K} \oplus \alpha \mathbf{K}$. Then $\pi_{l}(\Omega)=$ $\left\{z_{l} \in \mathbf{K} \oplus \alpha \mathbf{K}: R_{1} \leq\left|z_{l}-\xi_{l}\right| \leq R_{2}\right\}$. To prove the theorem consider $f$ by each variable $z_{l}$. That is, consider $z=z_{l}$ and $m=1$. Let $R_{3}$ and $R_{4}$ be such that $R_{1}<R_{3}<R_{4}<R_{2}$ and $z \in W \subset \Omega$, where $W=\left\{z \in \mathbf{K} \oplus \alpha \mathbf{K}: R_{3} \leq|z-\xi| \leq R_{4}\right\}$. In view of Theorems 2.8 and 2.20(iv), (vi),

$$
\begin{aligned}
f(z) & =C(\alpha)^{-1}{ }_{\partial W} P^{n}\left[f(\zeta)(\zeta-z)^{-1} d \zeta\right] \\
& =C(\alpha)^{-1} \partial_{c} B_{R_{4}} P^{n}\left[f(\zeta)(\zeta-z)^{-1} d \zeta\right]-C(\alpha)^{-1} \partial_{c} B_{R_{3}} P^{n}\left[f(\zeta)(\zeta-z)^{-1} d \zeta\right],
\end{aligned}
$$

since $W$ is the union $W_{1} \cup W_{2}, \operatorname{dim}_{\mathbf{K}}\left(W_{1} \cap W_{2}\right)=1$, where $W_{1}$ and $W_{2}$ satisfy Theorems 2.8 and 2.20. The part of the path $\gamma_{1,2}$ in $W_{1} \cap W_{2}$ joining $\partial_{c} B_{R_{4}}$ with $\partial_{c} B_{R_{3}}$ and forming two paths $\gamma_{1}$ and $\gamma_{2}$ affine homotopic to points in $W_{1}$ and $W_{2}, \gamma_{1} \subset W_{1}, \gamma_{2} \subset W_{2}$, such that $\gamma_{1,2}$ goes twice in two opposite directions, gives (2.35). For each $\zeta \in \partial_{c} B_{R_{4}}$ we have $\left|(z-\xi)(\zeta-\xi)^{-1}\right|<1$, hence $(\zeta-z)^{-1}=\sum_{k=0}^{\infty}(z-\xi)^{k}(\zeta-\xi)^{-k-1}$ and inevitably

$$
\partial_{c} B_{R_{4}} P^{n}\left[f(\zeta)(\zeta-z)^{-1} d \zeta\right]=\sum_{k=0}^{\infty} a_{k}(z-\xi)^{k},
$$

where $a_{k}=C(\alpha)^{-1} \partial_{c} B_{R_{4}} P^{n}\left[f(\zeta)(\zeta-\xi)^{-k-1} d \zeta\right]$ for each $0 \leq k \in \mathbb{Z}$.

If $\zeta \in \partial_{c} B_{R_{3}}$, then $\left|(\zeta-\xi)(z-\xi)^{-1}\right|<1$ and $(\zeta-z)^{-1}=-\sum_{k=1}^{\infty}(\zeta-\xi)^{k-1}(z-\xi)^{-k}$, hence due to continuity of $P^{n}-C(\alpha)^{-1} \partial_{c} B_{R_{3}} P^{n}\left[f(\zeta)(\zeta-z)^{-1} d \zeta\right]=\sum_{k=1}^{\infty} a_{-k}(z-\xi)^{-k}$, where $a_{-k}=C(\alpha)^{-1} \partial_{c} B_{R_{3}} P^{n}\left[f(\zeta)(\zeta-\xi)^{k-1} d \zeta\right]$ for each $k \geq 1$. In view of Theorem 2.8 we get (2.34).

Definitions 2.27. A point $z \in A(\mathbf{K} \oplus \alpha \mathbf{K})$ is called an isolated critical point of a function $f$ if there exists a set $B(\mathbf{K} \oplus \alpha \mathbf{K}, z, R) \backslash\{z\}$ for $z \neq A$, and $\{\zeta \in \mathbf{K} \oplus \alpha \mathbf{K}: R<|\zeta|<\infty\}$ for $z=A$, on which $f$ is $(q, n)$-antiderivationally holomorphic. An isolated critical point $z$ of the function $f$ is called removable if there exists a $\operatorname{limit}_{\zeta \rightarrow z} f(\zeta)=g \in Y$; it is called a pole if there exists $\lim _{\zeta \rightarrow z}\|f(\zeta)\|=\infty$; it is called an essentially critical point if there exists neither finite nor infinite limit point, when $\zeta$ tends to $z$.

Theorem 2.28. Let $f$ satisfy Theorem 2.20(i) on $\Omega \backslash\{z\}$. A point $z \in \mathbf{K} \oplus \alpha \mathbf{K}$ is removable if and only if decomposition (2.33) does not contain the main part

$$
f(\zeta)=\sum_{k=0}^{\infty} a_{k}(\zeta-z)^{k} .
$$

Theorem 2.29. Let $f$ satisfy Theorem 2.20(i) on $\Omega \backslash\{z\}$. An isolated critical point $z \in$ $\mathbf{K} \oplus \alpha \mathbf{K}$ is a pole if and only if the main part of the series (2.33) contains only a finite and 
positive number of nonzero terms

$$
f(\zeta)=\sum_{k=-N}^{\infty} a_{k}(\zeta-z)^{k}, \quad N>0 .
$$

Theorem 2.30. Let $f$ satisfy Theorem 2.20(i) for $Y=\mathbf{K}(\alpha)$ on $\Omega \backslash\{z\}$. An isolated critical point $z$ of $f$ is essentially critical if and only if the main part of the series (2.33) in a neighborhood of $z$ contains an infinite family $\left\{a_{k} \neq 0: k<0\right\}$. If $z$ is an essentially critical point of $f$, $r=2$, that is, $\mathbf{K}(\alpha)=\mathbf{K} \oplus \alpha \mathbf{K}$, then for each $\xi \in A \mathbf{K}(\alpha)$ there exists a sequence $\left\{z_{n}: n \in \mathbb{N}\right\}$, $\lim _{n \rightarrow \infty} z_{n}=z$ such that $\lim _{n \rightarrow \infty} f\left(z_{n}\right)=\xi$.

The proof of the previous three theorems is analogous to the classical case (see, e.g., [23, Section II.7], [24]) due to Theorems 2.20 and 2.26.

Definition 2.31. Let $f \in C^{(q, n)}(\Omega, Y)$ and $B:=B(\mathbf{K} \oplus \alpha \mathbf{K}, z, R) \subset \Omega, 0<R<\infty, f$ is $(q, n)$ antiderivationally holomorphic on $B \backslash\{z\}$, then

(i) $\operatorname{res}_{z} f:=C(\alpha)^{-1} \partial_{c} B P^{n}[f(\zeta) d \zeta]$

is called the residue of $f$, where $Y$ is a Banach space over $\mathbf{L}$ such that $\mathbf{K}(\alpha) \subset \mathbf{L}$.

THeовем 2.32. Let $f$ satisfy Theorem 2.20(i) on $\Omega \backslash \bigcup_{l=1}^{v}\left\{z_{l}\right\}$ such that $\partial \Omega$ does not contain critical points $z_{l}$ of $f$ and all of them are encompassed by $\partial \Omega, \nu \in \mathbb{N}$. Then

(i) $\partial_{\Omega} P^{n}[f(\zeta) d \zeta]=C(\alpha) \sum_{z_{l} \in \Omega} \operatorname{res}_{z_{l}} f$,

where $\operatorname{res}_{z_{l}} f$ is independent of $n$ and $R$ in Definition 2.31,

(ii) $\operatorname{res}_{z_{l}} f=a_{-1}, a_{k}$ is as in (2.33).

Proof. In view of Theorems 2.4 and 2.8, $C(\alpha)=C_{n}(\alpha)$ is independent of $n$ and $\operatorname{res}_{z_{l}} f$ is independent of $n$ and $R$. From (2.34), (ii) follows.

Definition 2.33. Let $f \in C^{(q, n)}(\Omega, Y)$ and let $A \in \Omega \subset A(\mathbf{K} \oplus \alpha \mathbf{K})$ be the isolated critical point of $f$. Put

$$
\operatorname{res}_{A} f:=-C(\alpha)^{-1}{ }_{\partial B} P^{n}[f(\zeta) d \zeta]
$$

Theorem 2.34. Let $f$ satisfy Theorem 2.20(i) on $(\mathbf{K} \oplus \alpha \mathbf{K}) \backslash \bigcup_{l=1}^{v}\left\{z_{l}\right\}$, then

(i) $\operatorname{res}_{A} f+\sum_{l=1}^{v} \operatorname{res}_{z_{l}} f=0$.

Proof. Take a ball $B_{R}:=B(\mathbf{K} \oplus \alpha \mathbf{K}, 0, R)$ of sufficiently large $0<R_{0}<R<\infty$ such that it contains all $\left\{z_{l}: l=1, \ldots, \nu\right\}, \Omega=A(\mathbf{K} \oplus \alpha \mathbf{K})$, and $\kappa(\Omega) \subset \mathbf{K} \oplus \alpha \mathbf{K}$ (see Section 2.2.4). In view of Theorem 2.32,

(ii) $\partial_{c} B_{R} P^{n}[f(\zeta) d \zeta]=\sum_{l=1}^{v} \operatorname{res}_{z_{l}} f$

and it is independent of $R$ for each $R>R_{0}, R<\infty$. In accordance with Definition 2.33 and Theorem 2.8,

$$
\text { (iii) } \partial_{c} B_{R} P^{n}[f(\zeta) d \zeta]=-\operatorname{res}_{A} f \text {. }
$$

Therefore, from (ii) and (iii), (i) follows.

Definitions 2.35. Let $f \in C^{(q, n)}(\Omega, \mathbf{K}(\alpha))$ and let $f$ be $(q, n)$-antiderivationally holomorphic on $B(\mathbf{K} \oplus \alpha \mathbf{K}, z, R) \backslash\{z\}$, where $\Omega \subset \mathbf{K} \oplus \alpha \mathbf{K}, f(z) \neq 0$. Then $\operatorname{res}_{z} f^{\prime}(z) / f(z)$ is called 
the logarithmic residue of $f$ at the point $z$. We count each zero and pole of $f$ a number of times equal to its order.

A function $f$ is called $(q, n)$-antiderivationally meromorphic if it is $(q, n)$-antiderivationally holomorphic on $\Omega$ beside a set of poles.

THEOREM 2.36. Let $f$ be $(q, n)$-antiderivationally meromorphic on $\Omega$ and let $\log (f)$ satisfy Theorem 2.20(i) on $\Omega \backslash \bigcup_{l=1}^{v}\left\{z_{l}\right\}$, where $z_{l}$ is the pole of $f$ for each $l=1, \ldots, \nu$, all zeros and poles of $f$ are encompassed by $\partial \Omega, Y=\mathbf{K}(\alpha)$. Then

$$
N-P=C(\alpha)^{-1}{ }_{\partial \Omega} P^{n}[d \log (f(\zeta))]
$$

where $N$ and $P$ denote total numbers of zeros and poles in $\Omega$.

Proof. Since $\Omega$ is compact, then $N$ and $P$ are finite. In view of Theorem 2.26,

$$
C(\alpha)^{-1}{ }_{\partial \Omega} P^{n}[d \log (f(\zeta))]=\sum_{l=1}^{\nu} \operatorname{res}_{z_{l}} \log (f)+\sum_{l=1}^{\mu}{\operatorname{res} \xi_{l}}_{\log }(f)
$$

where $z_{l}$ is the pole of $f$ and $\xi_{l}$ is the zero of $f$ for each $l$. On the other hand,

$$
\begin{aligned}
\frac{f^{\prime}(z)}{f(z)} & =\left[k\left(z-\xi_{l}\right)^{k-1} \phi(z)+\left(z-\xi_{l}\right)^{k} \phi^{\prime}(z)\right] \frac{\left(z-\xi_{l}\right)^{-k}}{\phi(z)} \\
& =\left(z-\xi_{l}\right)^{-1} \frac{\left[k \phi(z)+\left(z-\xi_{l}\right) \phi^{\prime}(z)\right]}{\phi(z)}
\end{aligned}
$$

where $f(z)=\left(z-\xi_{l}\right)^{k} \phi(z), k=k_{l}$ is the order of zero $\xi_{l}$, and $\phi(z) \neq 0$ in a neighborhood of $\xi_{l}$. Therefore,

$$
\operatorname{res}_{\xi_{l}} \log (f)=k_{l}, \quad \operatorname{res}_{z_{l}} \log (f)=-s_{l},
$$

where $s_{l}$ is the order of pole $z_{l}$. Hence, from (2.41), (2.43), (2.40) follows.

Theorem 2.37. Let $\Omega$ be a clopen compact subset in $B\left((\mathbf{K} \oplus \alpha \mathbf{K})^{m}, y, R\right), 0<R<p^{1 /(1-p)}$. Suppose

(i) $f_{j}\left(z_{1}, \ldots, z_{l-1}, z_{l}+\operatorname{Exp}(\eta), z_{l+1}, \ldots, z_{m}\right):=\psi_{j, l}(\eta): \omega_{l, \epsilon} \rightarrow Y$ belongs to ${ }_{S} C^{(q+1, n-1)}\left(\omega_{l, \epsilon}, Y\right)$ for each $j, l=1, \ldots, m$ and each $z=\left(z_{1}, \ldots, z_{m}\right) \in \Omega$, where $\omega_{l}=\left\{\eta \in \mathbf{K}(\alpha):\left(z_{1}, \ldots, z_{l-1}, z_{l}+\right.\right.$ $\left.\left.\operatorname{Exp}(\eta), z_{l+1}, \ldots, z_{m}\right) \in \Omega\right\}, \omega_{l, \epsilon}=\omega_{l} \backslash \log \left(B\left(\mathbf{K}(\alpha), z_{l}, \epsilon\right)\right), \epsilon=\epsilon_{k}, 0<\epsilon_{k}$ for each $k \in \mathbb{N}$, $\lim _{k \rightarrow \infty} \epsilon_{k}=0,0 \leq q \in \mathbb{Z}, 1 \leq n \in \mathbb{N}$, and $Y$ is a Banach space over $\mathbf{L}$ such that $\mathbf{K}(\alpha) \subset \mathbf{L}$. Assume that

(1) $\partial f_{j} / \partial \bar{z}_{l}=\partial f_{l} / \partial \bar{z}_{j}$ for each $j, l=1, \ldots, m$.

Then there exists $u \in C^{(q, n-1)}(\Omega, \mathbf{K}(\alpha))$ such that

(2) $\partial u(z) / \partial \bar{z}_{j}=f_{j}(z)$ for each $j=1, \ldots, m$ and each $z \in \widetilde{\Omega}$ (see Theorem 2.20).

Proof. Define

(3) $u(z):=C(\alpha)^{-1} \sum_{j=1}^{m} \bar{z}_{j \partial \Omega_{j}} P^{n}\left[f_{j}\left(z_{1}, \ldots, z_{j-1}, \zeta, z_{j+1}, \ldots, z_{m}\right)\left(\zeta-z_{j}\right)^{-1} d \zeta\right]-$ $C(\alpha)^{-1}{ }_{\Omega}^{n}\left[f_{1}\left(\zeta, z_{2}, \ldots, z_{m}\right)\left(\zeta-z_{1}\right)^{-1} d \bar{\zeta} \wedge d \zeta\right]$. 
Hence

$$
\begin{aligned}
u(z)= & C(\alpha)^{-1} \sum_{j=1}^{m} \bar{z}_{j \partial \Omega_{j}} P^{n}\left[f_{j}\left(z_{1}, \ldots, z_{j-1}, \zeta, z_{j+1}, \ldots, z_{m}\right)\left(\zeta-z_{j}\right)^{-1} d \zeta\right] \\
& +C(\alpha)^{-1} U^{2} P^{n}\left[\left(\chi_{\Omega_{1}} f_{1}\right)\left(z_{1}-\eta, z_{2}, \ldots, z_{m}\right) \eta^{-1} d\left(\overline{z_{1}-\eta}\right) \wedge d\left(z_{1}-\eta\right)\right],
\end{aligned}
$$

where $\eta:=z_{1}-\zeta$ and we can take $U=B(\mathbf{K}, 0, R)$ such that $U^{2}+U^{2}=U^{2}$ and $U^{2}$ is the additive group, $\Omega_{j}:=\left\{\xi \in U^{2}:\left(z_{1}, \ldots, z_{j-1}, \xi, z_{j+1}, \ldots, z_{m}\right) \in \Omega\right\}$. Therefore, $u \in C^{(q, n-1)}(\Omega, Y)$. Then

$$
\begin{aligned}
\frac{\partial u}{\partial \bar{z}_{j}}= & C(\alpha)^{-1} \partial_{\Omega_{j}} P^{n}\left[f_{j}\left(z_{1}, \ldots, z_{j-1}, \zeta, z_{j+1}, \ldots, z_{m}\right)\left(\zeta-z_{j}\right)^{-1} d \zeta\right] \\
& -C(\alpha)^{-1} U^{2} P^{n}\left[\left(\chi_{\Omega_{1}} \partial \frac{f_{1}}{\partial \bar{z}_{j}}\left(\zeta, z_{2}, \ldots, z_{m}\right)\right)\left(\zeta-z_{1}\right)^{-1} d \bar{\zeta} \wedge d \zeta\right]
\end{aligned}
$$

In view of condition (1), formula (6) in the proof of Theorem 2.18, and

$$
\begin{aligned}
f_{j}(z)= & C(\alpha)^{-1}{ }_{\partial \Omega_{j}} P^{n}\left[f_{j}\left(z_{1}, \ldots, z_{j-1}, \zeta, z_{j+1}, \ldots, z_{m}\right)\left(\zeta-z_{j}\right)^{-1} d \zeta\right] \\
& -C(\alpha)^{-1} U^{2} P^{n}\left[\chi_{\Omega_{j}}\left(\frac{\partial f_{j}}{\partial \bar{\zeta}}\left(\zeta, z_{2}, \ldots, z_{m}\right)\right)\left(\zeta-z_{1}\right)^{-1} d \bar{\zeta} \wedge d \zeta\right]
\end{aligned}
$$

(see Theorem 2.4), (2) follows.

\section{Antiderivational representations of functions and differential forms}

3.1. Remark and notation. Let $\Omega$ be a clopen compact subset in $(\mathbf{K} \oplus \alpha \mathbf{K})^{m}$. Put

(1) $w(z, \zeta):=\sum_{j=1}^{m}(-1)^{j+1}\left(\zeta_{j}-z_{j}\right)^{-1} d \zeta_{j} \wedge_{l \neq j}\left[(\xi(\bar{\zeta}-\bar{z}))^{-1} d_{\bar{\zeta}} \xi_{l}(\bar{\zeta}-\bar{z}) \wedge(\xi(\zeta-z))^{-1}\right.$ $\left.\times d_{\zeta} \xi_{l}(\zeta-z)\right]$ for each $z \neq \zeta \in \Omega^{2}$, where $\left(\alpha^{\prime}\right)^{j} \neq \alpha^{t}$ for each $j=1, \ldots, q^{\prime} ; t=$ $1, \ldots, q, m \leq q^{\prime} \leq \tilde{m}\left(\alpha^{\prime}\right), r \leq q \leq \tilde{m}(\alpha)$ (see Sections 2.1 and 2.2.6); there are constants $0<\epsilon_{1}<\epsilon_{2}<\infty$ such that

(2) $\epsilon_{1}|\pi|^{-s}|\zeta| \leq|\log (\xi(\zeta))| \leq \epsilon_{2}|\pi|^{-s}|\zeta|$ and $\xi(\zeta) \neq 0$ for each $\zeta \in \Omega-z, \xi(0)=1$, where $z \in \Omega, \xi(\zeta) \in C^{(q, n)}\left(\Omega-z, \mathbb{C}_{\mathbf{p}}\right), \log (\xi(\zeta)) \in(\mathbf{K} \oplus \alpha \mathbf{K})^{m}$ for each $\zeta \in \Omega-z$; here the embeddings used are: $(\mathbf{K} \oplus \alpha \mathbf{K})^{m} \hookrightarrow(\mathbf{K}(\alpha))^{m} \hookrightarrow \mathbf{K}\left(\alpha, \alpha^{\prime}\right) \hookrightarrow \mathbb{C}_{\mathbf{p}}$;

(3) $\xi$ is such that $d_{\zeta} w(z, \zeta)=0$ on $\Omega \backslash\{z\}$;

(4) $s:=s(\zeta):=-\operatorname{ord}_{\mathrm{K}\left(\alpha, \alpha^{\prime}\right)}(\zeta)$ for each $\zeta \in \Omega-z, z_{j} \neq \zeta_{j}$ for each $j, \alpha^{\prime}$ is the root of 1 in $\mathbb{C}_{\mathbf{p}}$ such that $\mathbf{K}(\alpha)^{m}$ is embedded into $\mathbf{K}\left(\alpha, \alpha^{\prime}\right)=(\mathbf{K}(\alpha))\left(\alpha^{\prime}\right),|z|_{\mathbf{K}\left(\alpha, \alpha^{\prime}\right)}=$ $|\pi|^{-\operatorname{ord}_{\mathbf{K}\left(\alpha, \alpha^{\prime}\right)}(z)}, \pi$ is the same as in Section 2.1;

(5) $\lim _{l \rightarrow \infty} \partial_{c} B\left((\mathbf{K} \oplus \alpha \mathbf{K})^{m}, z,|\pi|^{l}\right) P^{n}[w(z, \zeta)]=: q_{m} \neq 0$. If $f$ is a 1 -form of class $C^{(0, n-1)}$, we define

(6) $\left(B_{\Omega}^{n} f\right)(z):=q_{m}^{-1}{ }_{\Omega}^{n}[f(\zeta) \wedge w(z, \zeta)]$ for each $z \in \Omega$ encompassed by $\partial \Omega$.

If $f \in C^{(0, n-1)}(\Omega, Y)$, we define

(7) $\left(B_{\partial \Omega}^{n} f\right)(z):=q_{m}^{-1} \zeta \in \partial \Omega P^{n}[f(\zeta) w(z, \zeta)]$ for each $z \in \Omega$ encompassed by $\partial \Omega$, where $Y$ is a Banach space over $\mathbf{L}$ such that $\mathbf{K}(\alpha) \subset \mathbf{L}$. 
TheORem 3.1. Let $\Omega$ be a clopen compact subset in $B\left((\mathbf{K} \oplus \alpha \mathbf{K})^{m}, y, R\right), 0<R<p^{1 /(1-p)}$, $B_{\Omega}^{n}, B_{\partial \Omega}^{n}$ given by Section 3.1, $f \in C^{(q+1, n-1)}(\Omega, Y)$. Then

(1) $f(z)=\left(B_{\partial \Omega}^{n} f\right)(z)-\left(B_{\Omega}^{n} \bar{\partial} f\right)(z)$ for each $z=\left(z_{1}, \ldots, z_{m}\right) \in \widetilde{\Omega}$ (see Theorem 2.20) such that

(2) $(f w)\left(z_{1}, \ldots, z_{l-1}, z_{l}+\operatorname{Exp}(\eta), z_{l+1}, \ldots, z_{m}\right)=: \widetilde{\psi}_{l}(\eta) \in{ }_{S} C^{(q+1, n-1)}\left(\omega_{l, \epsilon}, L(\Lambda \mathbf{K}(\alpha), Y)\right)$ for each $l=1, \ldots, m$ and each $\epsilon=\epsilon_{j}$, where

$$
\omega_{l}:=\left\{\eta \in \mathbf{K}(\alpha):\left(z_{1}, \ldots, z_{l-1}, z_{l}+\operatorname{Exp}(\eta), z_{l+1}, \ldots, z_{m}\right) \in \Omega\right\}
$$

$\omega_{l, \epsilon}:=\omega_{l} \backslash \log \left(B\left(\mathbf{K}(\alpha), z_{l}, \epsilon\right)\right), 0<\epsilon_{j}$ for each $j \in \mathbb{N}, \lim _{j \rightarrow \infty} \epsilon_{j}=0,0 \leq q \in \mathbb{Z}, 1 \leq$ $n \in \mathbb{N}$ (see Section 2.2.6).

Proof. Fix $z \in \tilde{\Omega}$. In the particular case $\xi(\bar{\zeta}-\bar{z})=\operatorname{Exp}\left(\pi^{-s}(\bar{\zeta}-\bar{z})\right)$ properties (2), (3) in Section 3.1 are satisfied and $q_{m}=C(\alpha) m(2 \alpha)^{m-1}$ due to formulas (2.2) and (2.3), Section 2.2.4, and Theorem 2.4, since $d \bar{z} \wedge d z=2 \alpha d x \wedge d y$ and $\left.{ }_{U} P^{n}[d x]\right|_{a} ^{b}=b-a$ for each $a, b \in U$, where $z=x+\alpha y, x, y \in U \subset \mathbf{K}$. Therefore, the family of such $\xi$ and $w$ satisfying conditions (1)-(5) in Section 3.1 is nonvoid. In view of (3) in Section 3.1, $d_{\zeta} w(z, \zeta)$ $=0$ on $\Omega \backslash\{z\}$, hence $d(f(\zeta) w(z, \zeta))=\bar{\partial} f(\zeta) \wedge w(z, \zeta)$ on $\Omega \backslash\{z\}$, since $\partial f(\zeta) \wedge w(z, \zeta)$ $=0$ on $\Omega \backslash\{z\}$. From Corollary 2.3 it follows that there exists $\delta>0$ such that for each $0<\epsilon<\delta, \epsilon \in \Gamma_{\mathbf{K}}$, there is the inclusion $B(\epsilon):=B\left((\mathbf{K} \oplus \alpha \mathbf{K})^{m}, z, \epsilon\right) \subset \Omega$ and the following equality is satisfied:

$$
\zeta \in \partial_{c} B(\epsilon) P^{n}[f(\zeta) w(z, \zeta)]={ }_{\partial \Omega} P^{n}[f(\zeta) w(z, \zeta)]-{ }_{\Omega(\epsilon)} P^{n}[\bar{\partial} f(\zeta) \wedge w(z, \zeta)]
$$

for each $z \in \widetilde{\Omega}$ and satisfying Section 2.2.4(i-iii), where $\Omega(\epsilon):=\{\zeta \in \Omega:|\zeta-z| \geq \epsilon\}$. In the particular case, $\xi(\bar{\zeta}-\bar{z})=\operatorname{Exp}\left(\pi^{-s}(\bar{\zeta}-\bar{z})\right)$ due to Section 2.2.4:

$$
\begin{gathered}
\zeta \in \partial_{c} B(\epsilon) P^{n}[f(\zeta) w(z, \zeta)] \\
=\pi^{-2(m-1) s}{ }_{\zeta \in \partial_{c} B(\epsilon)} P^{n}\left[f(\zeta) \sum_{j=1}^{m}(-1)^{j+1}\left(\zeta_{j}-z_{j}\right)^{-1} d \zeta_{j} \wedge l \neq j\right. \\
=\pi^{-2(m-1) s}(2 \alpha)^{m-1}\left(\sum_{j=1}^{m}(-1)^{j+1}{ }_{B\left((\mathbf{K} \oplus \alpha \mathbf{K})^{m-1}, z^{\prime}, \epsilon\right)}\right. \\
\left.\times P^{n}\left\{\zeta_{l}\right)\right] \\
\wedge_{l \neq j}\left(d x_{c} B\left(\mathbf{K} \oplus \alpha \mathbf{K}, z_{j}, \epsilon\right)\right. \\
\left.\left.\left.\wedge_{l-1} \wedge d x_{2 l}\right)\right\}\right),
\end{gathered}
$$

where $z^{\prime}=\left(z_{1}, \ldots, z_{j-1}, z_{j+1}, \ldots, z_{m}\right), x_{2 l-1}, x_{2 l} \in U, z_{l}=x_{2 l-1}+\alpha x_{2 l}$ for each $l=1, \ldots, m$. Therefore, there exists

$$
\lim _{\epsilon \rightarrow 0} \zeta \in \partial_{c} B(\epsilon) P^{n}[f(\zeta) w(z, \zeta)]=f(z) q_{m}
$$

due to (2) and (5) in Section 3.1, since there exists $C=$ const $>0, C<\infty$, such that

$$
\left|{ }_{B(\epsilon)} P^{n}[(f(\zeta)-f(z)) w(z, \zeta)]\right| \leq C \epsilon\|f(\zeta)-f(z)\|_{C^{(0, n-1)}(B(\epsilon), Y)}
$$

for each $0<\epsilon<\delta$. 
Corollary 3.2. Let $\Omega$ and $f$ be as in Theorem 3.1 and let $f$ be derivationally $(q, n)$ holomorphic on $\Omega$, then

$$
f(z)=\left(B_{\partial \Omega}^{n} f\right)(z) \quad \text { for each } z \in \widetilde{\Omega}
$$

(see Theorem 2.20).

Remark 3.3. For $m=1$ formula (1) in Section 3.1 and (2.10) are equivalent; they are the non-Archimedean analogs of the Martinelli-Bochner and Cauchy-Green formulas, respectively (cf. the classical complex case in [8]).

3.2. Definitions and notations. Consider a clopen compact $\Omega \subset(\mathbf{K} \oplus \alpha \mathbf{K})^{m}$ and a $C^{(q, n+1)}$ function $v: \Omega \times(\partial \Omega)^{\delta} \rightarrow(\mathbf{K} \oplus \alpha \mathbf{K})^{m}, v=\left(v_{1}, \ldots, v_{m}\right), 0<\delta<\infty, v=v(z, \zeta), z \in \Omega, \zeta \in$ $(\partial \Omega)^{\delta}, \Psi^{\epsilon}:=\{z \in X: d(z, \Psi)<\epsilon\}$ for a topological space $X$ with a metric $d$ and a subset $\Psi \subset X, d(z, \Psi):=\inf _{x \in \Psi} d(z, x), 0<\epsilon, 0 \leq q \in \mathbb{Z}, 1 \leq n \in \mathbb{N}$. Suppose

$$
\tilde{\phi}(s):=-\operatorname{ord}_{\mathbf{K}\left(\alpha, \alpha^{\prime}\right)} v(z, \zeta), \quad s=-\operatorname{ord}_{\mathbf{K}\left(\alpha, \alpha^{\prime}\right)}(\bar{\zeta}-\bar{z})
$$

such that

(1) $\lim _{s \rightarrow \infty} \tilde{\phi}(s)=\infty$

for each $z \in \Omega$ and $\zeta \in(\partial \Omega)^{\delta}$. Put

$$
\eta^{v}(z, \zeta, \lambda):=\left(1-\frac{\lambda}{\beta}\right) \xi(v(z, \zeta))+\frac{\lambda \xi(\bar{\zeta}-\bar{z})}{\beta},
$$

where $\lambda \in B(\mathbf{K}, 0,1)$. Impose the condition

(2) $\wedge_{k=1}^{m} d_{\zeta} v_{k}(z, \zeta) \wedge_{j=1}^{m} d \zeta_{j} \neq 0$ and $\eta^{v}(z, \zeta, \lambda) \neq 0$

for each $z \in \Omega, \zeta \in(\partial \Omega)^{\delta}$, and $\lambda \in B(\mathbf{K}, 0,1)$. Let also

(3)

$$
\begin{array}{r}
\psi(z, \zeta):=\sum_{j=1}^{m}(-1)^{j+1}\left(\zeta_{j}-z_{j}\right)^{-1} d \zeta_{j} \wedge_{k \neq j}\left[(\xi(v(z, \zeta)))^{-1} d_{\zeta} \xi_{k}(v(z, \zeta))\right. \\
\left.\wedge(\xi(\zeta-z))^{-1} d_{\zeta} \xi_{k}(\zeta-z)\right] .
\end{array}
$$

If $f \in C^{(0, n-1)}(\partial \Omega, Y)$, we set

(4) $\left(L_{\partial \Omega}^{v, n} f\right)(z):=q_{m}^{-1} \zeta \in \partial \Omega P^{n}[f(\zeta) \psi(z, \zeta)]$

for each $z \in \Omega$, where $Y$ is a Banach space over $\mathbf{L}$ such that $\mathbf{K}(\alpha) \subset \mathbf{L}$. Put also

(5) $\gamma(z, \zeta, \lambda):=\sum_{j=1}^{m}(-1)^{j+1}\left(\zeta_{j}-z_{j}\right)^{-1} d \zeta_{j} \wedge_{k \neq j}\left[\left(\eta^{v}(z, \zeta, \lambda)\right)^{-1}\left(\bar{\partial}_{z, \zeta}+d_{\lambda}\right) \eta_{k}^{v}(z, \zeta, \lambda) \wedge\right.$ $\left.(\xi(\zeta-z))^{-1} d_{\zeta} \xi_{k}(\zeta-z)\right]$

for each $z \in \Omega, \zeta \in(\partial \Omega)^{\delta}, \lambda \in B(\mathbf{K}, 0,1)$. If $f$ is a $C^{(0, n-1)}-1$-form on $\partial \Omega$, put

(6) $\left(R_{\partial \Omega}^{v, n} f\right)(z):=q_{m}^{-1} \zeta \in \partial \Omega, \lambda \in B(\mathrm{~K}, 0,1) P^{n}[f(\zeta) \wedge \gamma(z, \zeta, \lambda)]$

for each $z \in \Omega$. Suppose that $v$ is such that 
(7) $d_{z, \zeta} \psi(z, \zeta)=0$ for each $\zeta \neq z$. In particular, if $v(z, \zeta)=\bar{\zeta}-\bar{z}$, then

$$
L_{\partial \Omega}^{v, n} f=B_{\partial \Omega}^{n} f, \quad R_{\partial \Omega}^{v, n} f=0,
$$

since $\gamma$ is the $(m, m)$-form by $(\zeta, \bar{\zeta})$ for $v(z, \zeta)=\bar{\zeta}-\bar{z}$.

THeOREM 3.4. Let $\Omega$ be a clopen compact subset in $(\mathbf{K} \oplus \alpha \mathbf{K})^{m}$ and let $v(z, \zeta), L_{\partial \Omega}^{v, n}$, and $R_{\partial \Omega}^{v, n}$ be given by Section 3.2, $f \in C^{(q+1, n-1)}(\Omega, Y)$. Then

(1) $f(z)=\left(L_{\partial \Omega}^{v, n} f\right)(z)-\left(R_{\partial \Omega}^{v, n} \bar{\partial} f\right)(z)-\left(B_{\Omega}^{n} \bar{\partial} f\right)(z)$ for each $z \in \widetilde{\Omega}$ (see Theorem 2.20) such that

(2) $(f \gamma)\left(z_{1}, \ldots, z_{l-1}, z_{l}+\operatorname{Exp}(\eta), z_{l+1}, \ldots, z_{m}\right)=: \widetilde{\psi}_{l}(\eta) \in{ }_{s} C^{(q+1, n-1)}\left(\omega_{l, \epsilon}, L(\Lambda \mathbf{K}(\alpha), Y)\right)$ for each $l=1, \ldots, m, \epsilon=\epsilon_{j}$, where

$$
\begin{gathered}
\omega_{l}:=\omega_{l}(z):=\left\{\eta \in \mathbf{K}(\alpha):\left(z_{1}, \ldots, z_{l-1}, z_{l}+\operatorname{Exp}(\eta), z_{l+1}, \ldots, z_{m}\right) \in \Omega\right\}, \\
\omega_{l, \epsilon}:=\omega_{l} \backslash \log \left(B\left(\mathbf{K}(\alpha), z_{l}, \epsilon\right)\right), \quad 0<\epsilon_{j} \in \Gamma_{\mathbf{K}},
\end{gathered}
$$

for each $j \in \mathbb{N}, \lim _{j \rightarrow \infty} \epsilon_{j}=0,0 \leq q \in \mathbb{Z}, 1 \leq n \in \mathbb{N}$ (see Section 2.2.6).

Proof. The use of Theorem 3.1 reduces the proof to that of the formula

(3) $\left(R_{\partial \Omega}^{v, n} \bar{\partial} f\right)(z)=\left(L_{\partial \Omega}^{v, n} f\right)(z)-\left(B_{\partial \Omega}^{n} f\right)(z)$

for each $z \in \widetilde{\Omega}$ and satisfying condition (2). In view of (2) in Section 3.1 and (7) in Section 3.2 we have $d_{\zeta, \lambda} \gamma(z, \zeta, \lambda)=0$, since $d_{\zeta, \lambda}\left[d_{\zeta, \lambda}\left(\eta^{v}\right)\right]=0$. Therefore, $d_{\zeta, \lambda}[f(\zeta) \gamma(z, \zeta, \lambda)]$ $=(\bar{\partial} f(\zeta)) \wedge \gamma(z, \zeta, \lambda)$, since $(\partial f) \wedge \gamma=0$. From (3) and (5) in Section 3.2 it follows that

$$
\begin{aligned}
\left.\gamma(z, \zeta, \lambda)\right|_{\lambda=0} & =\psi(z, \zeta), \\
\left.\gamma(z, \zeta, \lambda)\right|_{\lambda=\beta}= & \sum_{j=1}^{m}(-1)^{j+1}\left(\zeta_{j}-z_{j}\right)^{-1} d \zeta_{j} \\
& \wedge_{k \neq j}\left[(\xi(\bar{\zeta}-\bar{z}))^{-1} d_{\bar{\zeta}} \xi_{k}(\bar{\zeta}-\bar{z}) \wedge(\xi(\zeta-z))^{-1} d_{\zeta} \xi_{k}(\zeta-z)\right] .
\end{aligned}
$$

Mention that $\lambda=\left.P^{n} 1\right|_{0} ^{\lambda}$, hence $\lambda \in{ }_{P} C^{(q, n)}(B(\mathbf{K}, 0,1), \mathbf{K})$. Then for degree reasons

$$
\zeta \in \partial \Omega P^{n}\left[\left.f \gamma\right|_{\lambda=\beta}\right]=\zeta \in \partial \Omega P^{n}[f w],
$$

where $w$ is given by (1) in Section 3.1, $\operatorname{dim}_{K} \Omega=2 m, \operatorname{dim}_{K} \partial \Omega=2 m-1$. Then

$$
\begin{aligned}
q_{m}^{-1} \zeta \in \partial \Omega, \lambda \in B & P^{n}\left\{d_{\zeta, \lambda}[f(\zeta) \gamma(z, \zeta, \lambda)]\right\} \\
& =q_{m}^{-1} \zeta \in \partial \Omega, \lambda \in B \\
& =\left(R_{\partial \Omega}^{v, n} \bar{\partial} f\right)(z)
\end{aligned}
$$

for each $z \in \widetilde{\Omega}$, where $B:=B(\mathbf{K}, 0,1)$. On the other hand,

$$
\begin{aligned}
\partial((\partial \Omega) \times B) & =(-1)^{2 m-1}((\partial \Omega) \times\{\beta\}-(\partial \Omega) \times\{0\}) \\
& =-(\partial \Omega) \times\{\beta\}+(\partial \Omega) \times\{0\} .
\end{aligned}
$$


In view of Corollary 2.3,

$$
(\partial \Omega) \times B P^{n}\left\{d_{\zeta, \lambda}[f \gamma]\right\}=-{ }_{\partial \Omega} P^{n}[f w]+{ }_{\partial \Omega} P^{n}[f \psi],
$$

hence formula (3) is accomplished.

Corollary 3.5. Let $f$ be as in Theorem 3.4 and $\bar{\partial} f=0$ on $\Omega$, then

$$
f(z)=\left(L_{\partial \Omega}^{v, n} f\right)(z)
$$

for each $z \in \widetilde{\Omega}$ (see Theorem 2.20) and satisfying Theorem 3.4(2).

3.3. Definitions and remarks. Let $\Omega$ be a clopen compact subset in $(\mathbf{K} \oplus \alpha \mathbf{K})^{m}$, and consider the following differential form:

(1)

$$
\begin{aligned}
\tilde{w}(z, \zeta):=\sum_{j=1}^{m}(-1)^{j+1}\left(\zeta_{j}-z_{j}\right)^{-1} d \zeta_{j} \wedge l \neq j & {\left[(\xi(\bar{\zeta}-\bar{z}))^{-1} \bar{\partial}_{\zeta, z} \xi_{l}(\bar{\zeta}-\bar{z})\right.} \\
& \left.\wedge(\xi(\zeta-z))^{-1} d \xi_{l}(\zeta-z)\right] .
\end{aligned}
$$

Let $M$ be a compact manifold over $\mathbf{K}$ and let $\phi: \Omega \rightarrow M \hookrightarrow(\mathbf{K}(\alpha))^{N}$ be a ${ }_{S} C^{(q+1, n-1)}$ diffeomorphism (see Section 2.2.5). Then the diffeomorphism $\phi_{*} w$ of the differential form $w$ is the differential form on $M$. Consider these differential forms on $M$ also and denote them by the same notation, since $\left\{\phi\left(\zeta_{j}\right): j\right\}$ are coordinates in $M$. Therefore, Theorems 3.1, 3.4 and Corollaries 3.2, 3.5 are true for $M$, also because of Theorem 2.2 and Corollary 2.3, where $\widetilde{M}:=\phi^{-1}(\widetilde{\Omega})$ (see Section 2.2.5 and Theorem 2.20). If $f$ is a $C^{(0, n-1)}$-differential form on $M$, then we define

(2) $\left(B_{M}^{n} f\right)(z):=q_{m}^{-1} \zeta \in M P^{n}[f(\zeta) \wedge \widetilde{w}(z, \zeta)]$ for each $z \in M$ encompassed by $\partial M$. If $f$ is a $C^{(0, n-1)}$-differential form on $M$, then we define

(3) $\left(B_{\partial M}^{n} f\right)(z):=q_{m}^{-1} \zeta \in \partial M P^{n}[f(\zeta) \wedge \widetilde{w}(z, \zeta)]$ for each $z \in M$ encompassed by $\partial M$. Write $\widetilde{w}$ as

(4) $\widetilde{w}(z, \zeta)=\sum_{t=0}^{m-1} \Upsilon_{t}(z, \zeta)$, where $\Upsilon_{t}$ is of bidegree $(0, t)$ in $z$ and of bidegree $(m, m-$ $t-1)$ in $\zeta$. Decompose $f$ as follows:

(5) $f=\sum_{l+s=\operatorname{deg}(f)} f_{(l, s)}$, where $f_{(l, s)}$ is the $(l, s)$-form on $M$. Then $f_{(l, s)}(\zeta) \wedge_{j=1}^{m} d \zeta_{j}=0$ for each $l>0$, hence $B_{M}^{n} f=B_{M}^{n} f_{(0, \operatorname{deg}(f))}$. On the other hand,

$$
\begin{aligned}
f(\zeta) \wedge \Upsilon_{t}(z, \zeta)=0 & \text { if } \operatorname{deg}(f)>q+1, \\
\zeta \in M P^{n}\left[f(\zeta) \wedge \Upsilon_{t}(z, \zeta)\right]=0 & \text { when } \operatorname{deg}(f)<q+1,
\end{aligned}
$$

by the definition of the antiderivation. Therefore,

(6) $B_{M}^{n} f=\zeta_{\zeta M} P^{n}\left[f_{(0, \operatorname{deg}(f))}(\zeta) \wedge \Upsilon_{\operatorname{deg}(f)-1}(z, \zeta)\right]$ for $1 \leq \operatorname{deg}(f) \leq m$,

(7) $B_{M}^{n} f=0$ for $\operatorname{deg}(f)=0$ or $\operatorname{deg}(f)>m$, similarly,

(8) $B_{\partial M}^{n} f=\zeta \in \partial M P^{n}\left[f_{(0, \operatorname{deg}(f))}(\zeta) \wedge \Upsilon_{\operatorname{deg}(f)}(z, \zeta)\right]$ for $0 \leq \operatorname{deg}(f) \leq m-1$,

(9) $B_{\partial M}^{n} f=0$ for $\operatorname{deg}(f) \geq m$,

hence $B_{M}^{n} f$ is of bidegree $(0, \operatorname{deg}(f)-1) ; P_{\partial M}^{n} f$ is of bidegree $(0, \operatorname{deg}(f))$. Using the notation of Section 3.2, define 
(10)

$$
\begin{array}{r}
\widetilde{\psi}(z, \zeta):=\sum_{j=1}^{m}(-1)^{j+1}\left(\zeta_{j}-z_{j}\right)^{-1} d \zeta_{j} \wedge_{k \neq j}\left[(\xi(v(z, \zeta)))^{-1} \bar{\partial}_{z, \zeta} \xi_{k}(v(z, \zeta))\right. \\
\left.\wedge(\xi(\zeta-z))^{-1} d_{\zeta} \xi_{k}(\zeta-z)\right],
\end{array}
$$

(11) $\tilde{\gamma}(z, \zeta, \lambda):=\sum_{j=1}^{m}(-1)^{j+1}\left(\zeta_{j}-z_{j}\right)^{-1} d \zeta_{j} \wedge_{k \neq j}\left[\left(\eta^{v}(z, \zeta, \lambda)\right)^{-1}\left(\bar{\partial}_{z, \zeta}+d_{\lambda}\right) \eta^{v}(z, \zeta, \lambda) \wedge\right.$ $\left.(\xi(\zeta-z))^{-1} d_{\zeta} \xi_{k}(\zeta-z)\right]$.

If $f \in C^{(0, n-1)}(\partial M, L(\Lambda \mathbf{K}(\alpha), Y))$, put

(12) $\left(L_{\partial M}^{v, n} f\right)(z):=q_{m}^{-1} \zeta \in \partial M P^{n}[f(\zeta) \wedge \widetilde{\psi}(z, \zeta)]$,

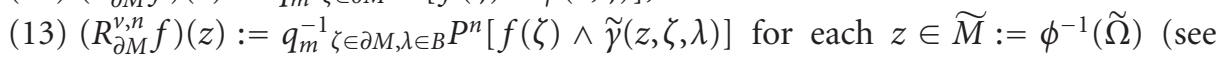
Section 2.2.5 and Theorem 2.20). There exists the decomposition

(14) $\tilde{\gamma}(z, \zeta, \lambda)=\sum_{t=0}^{m-1} \Upsilon_{t}^{v}(z, \zeta, \lambda)$, where $\Upsilon_{t}^{v}(z, \zeta, \lambda)$ is of bidegree $(0, t)$ in $z$ and $f$ is of bidegree $(m, m-t-1)$ in $(\zeta, \lambda)$. Let $f$ be a bounded differential form on $\partial M, f=$ $\sum f_{(l, s)}$, then $R_{\partial M}^{v, n} f=R_{\partial M}^{v, n} f_{(0, \operatorname{deg}(f))}$ and $f(\zeta) \wedge \Upsilon_{t}^{v}(z, \zeta, \lambda)=0$ if $\operatorname{deg}(f)>t+1$ by the definition of $P^{n},(\zeta, \lambda) \in \partial M \times B P^{n}\left[f(\zeta) \wedge \Upsilon_{t}^{v}(z, \zeta, \lambda)\right]=0$ if $\operatorname{deg}(f)<t+1$, hence

(15) $R_{\partial M}^{v, n} f={ }_{\zeta \in \partial M, \lambda \in B} P^{n}\left[f_{(0, \operatorname{deg}(f))}(\zeta) \wedge \Upsilon_{\operatorname{deg}(f)-1}^{v}(z, \zeta, \lambda)\right]$ if $1 \leq \operatorname{deg}(f) \leq m$,

(16) $R_{\partial M}^{v, n} f=0$ if $\operatorname{deg}(f)=0$ or $\operatorname{deg}(f)>m$. Similarly,

(17) $\widetilde{\psi}(z, \zeta)=\sum_{t=0}^{m-1} \Upsilon_{t}^{v}(z, \zeta)$, where $\Upsilon_{t}^{v}(z, \zeta)$ is of bidegree $(0, t)$ in $z$ and of bidegree $(m, m-t-1)$ in $\zeta$, hence

(18) $L_{\partial M}^{v, n} f={ }_{\zeta \in \partial M} P^{n}\left[f_{(0, \operatorname{deg}(f))} \wedge \Upsilon_{\operatorname{deg}(f)}^{v}(z, \zeta)\right]$ if $\operatorname{deg}(f) \leq m-1$,

(19) $L_{\partial M}^{v, n} f=0$ if $\operatorname{deg}(f) \geq m$. If $v(z, \zeta)=\bar{\zeta}-\bar{z}$, then $L_{\partial M}^{v, n} f=B_{\partial M}^{n} f$.

Theorem 3.6. Let $M$ be a compact manifold over $\mathbf{K}$ and let $B_{M}^{n}$ and $B_{\partial M}^{n}$ be given by Section 3.3. Suppose that $f$ is the $C^{(q+1, n-1)}-(0, t)-f o r m, 0 \leq t \leq m$. Then

(1) $(-1)^{t} f(z)=\left(B_{\partial M}^{n} f\right)(z)-\left(B_{M}^{n} \bar{\partial} f\right)(z)+\left(\bar{\partial} B_{M}^{n} f\right)(z)$ for each $z \in \widetilde{M}$ such that

(2) $(f \wedge \widetilde{w}) \circ \phi\left(z_{1}, \ldots, z_{l-1}, z_{l}+\operatorname{Exp}(\eta), z_{l+1}, \ldots, z_{m}\right)=: \widetilde{\psi}_{l}(\eta) \in{ }_{s} C^{(q+1, n-1)}\left(\omega_{l, \epsilon}\right.$, $L(\Lambda(\mathbf{K}(\alpha)), Y))$ for each $l=1, \ldots, m$ and each $\epsilon=\epsilon_{j}$, where $\omega_{l}:=\{\eta \in \mathbf{K}(\alpha)$ : $\left.\left(z_{1}, \ldots, z_{l-1}, z_{l}+\operatorname{Exp}(\eta), z_{l+1}, \ldots, z_{m}\right) \in \Omega\right\}, \omega_{l, \epsilon}:=\omega_{l} \backslash \log \left(B\left(\mathbf{K}(\alpha), z_{l}, \epsilon\right)\right), 0<\epsilon_{j}$ for each $j \in \mathbb{N}, \lim _{j \rightarrow \infty} \epsilon_{j}=0,0 \leq q \in \mathbb{Z}, 1 \leq n \in \mathbb{N}$.

Proof. Using the diffeomorphism $\phi$ it is possible to reduce the case to $\Omega \subset(\mathbf{K} \oplus \alpha \mathbf{K})^{m}$. If $q=0$, then by (7) in Section $3.3 B_{\Omega}^{n} f=0$ and $f=B_{\partial \Omega}^{n} f-B_{\Omega}^{n} \bar{\partial} f$ Theorem 3.1(1). Since (2) is satisfied, $v$ and $\xi \in C^{(q, n)}$, then $B_{\partial \Omega}^{n} f$ and $B_{\Omega}^{n} \bar{\partial} f$ are in ${ }_{S} C^{(q, n)}\left(\omega_{l, \epsilon}, L(\Lambda(\mathbf{K}(\alpha)), Y)\right)$ for each $l=1, \ldots, m, \epsilon=\epsilon_{j}$. From the definition of $B_{M}^{n}$ it follows that

$$
\begin{aligned}
\sup _{u}\left\|\bar{\Phi}^{u}\left(B_{\Omega}^{n} \bar{\partial} f\right)\left(z ; h_{1}^{\otimes u_{1}}, \ldots, h_{m}^{\otimes u_{m}} ; \zeta_{1}, \ldots, \zeta_{u}\right)-\bar{\Phi}^{u}\left(B_{\Omega}^{n} \bar{\partial} f\right)\left(y ; h_{1}^{\otimes u_{1}}, \ldots, h_{m}^{\otimes u_{m}} ; \zeta_{1}, \ldots, \zeta_{u}\right)\right\|_{C^{(q, n)}} \\
\quad \leq C_{1}\|f\|_{C^{(q, n)}}\left|1-\pi^{-2 s m}\right|
\end{aligned}
$$

where $s=s(\zeta-z), u=u_{1}+\cdots+u_{m}, 0 \leq u_{l} \leq n$, hence $\left(B_{M}^{n} \bar{\partial} f\right) \in C^{(q, n)}(M, L(\Lambda(\mathbf{K}(\alpha)), Y))$. Analogously, $B_{\partial M}^{n} f$ and $B_{M}^{n} f$ are in $C^{(q, n)}(M, L(\Lambda(\mathbf{K}(\alpha)), Y))$. It remains to prove that in 
the sense of distributions,

$$
\left(\bar{\partial} B_{\Omega}^{n} f\right)(z)=(-1)^{t} f(z)-\left(B_{\partial M}^{n} f\right)(z)+\left(B_{M}^{n} \bar{\partial} f\right)(z)
$$

for each $z \in \widetilde{\Omega}$ and satisfying condition (2). This means that for each ${ }_{S} C^{(q+1, n-1)}$-form $\nu$, $\operatorname{supp}(\nu) \subset \widetilde{\Omega}$, the following equality is satisfied:

(3) $(-1)_{\Omega}^{t} P^{n}\left[B_{\Omega}^{n} f \wedge \bar{\partial} \nu\right]=(-1)_{\Omega}^{t} P^{n}[f \wedge \nu]-{ }_{\Omega} P^{n}\left[B_{\partial \Omega}^{n} f \wedge \nu\right]+{ }_{\Omega} P^{n}\left[B_{\Omega}^{n}\left(\partial^{-} f\right) \wedge \nu\right]$. In view of formulas (6) and (8) in Section $3.3 B_{\partial \Omega}^{n} f$ and $B_{\Omega}^{n} \bar{\partial} f$ are of bidegree $(0, t)$ and $B_{\Omega}^{n} f$ is of bidegree $(0, t-1)$, we can assume that $\nu$ is of bidegree $(m, m-t)$. Then (3) takes the form

(4)

$$
\begin{aligned}
(-1)^{t}{ }_{(\zeta, z) \in \Omega^{2}} P^{n}[f(\zeta) \wedge \widetilde{w}(z, \zeta) \wedge \bar{\partial} \nu(z)] \\
=(-1)^{t}{ }_{z \in \Omega} P^{n}[f(z) \wedge \nu(z)] \\
\quad-(\zeta, z) \in(\partial \Omega) \times \Omega P^{n}[f(\zeta) \wedge \widetilde{w}(z, \zeta) \wedge \nu(z)] \\
\quad+(\zeta, z) \in \Omega^{2} P^{n}[\bar{\partial} f(\zeta) \wedge \widetilde{w}(z, \zeta) \wedge \nu(z)] .
\end{aligned}
$$

Put

(5) $\tilde{\theta}(z, \zeta):=\sum_{j=1}^{m}(-1)^{j+1}\left(\zeta_{j}-z_{j}\right)^{-1}\left(d \zeta_{j}-d z_{j}\right) \wedge_{k \neq j}\left[(\xi(\bar{\zeta}-\bar{z}))^{-1} \bar{\partial}_{\zeta, z} \xi_{k}(\bar{\zeta}-\bar{z})\right.$ $\left.\wedge(\xi(\zeta-z))^{-1} \partial_{\zeta, z} \xi_{k}(\zeta-z)\right]$, then from (1) and (3) in Section 3.1, and $d_{\zeta, z}=d_{\zeta}+$ $d_{z}$ it follows that

(6) $d_{\zeta, z} \tilde{\theta}(z, \zeta)=0$ for $\zeta \neq z$, since $\bar{\partial}\left(\zeta_{j}-z_{j}\right)=0, d_{\zeta}^{2}=0$.

Then all monomials in $\tilde{\theta}(z, \zeta)-\widetilde{w}(z, \zeta)$ contain at least one of the differentials $d z_{1}, \ldots, d z_{m}$. $v(z)$ of bidegree $(m, m-t)$ contains the factor $d z_{1} \wedge \cdots \wedge d z_{m}$, hence from (5), (6) it follows that

(7) $d_{\zeta, z}(\tilde{w}(z, \zeta) \wedge v(z))=d_{z, \zeta}(\tilde{\theta}(z, \zeta) \wedge \nu(z))=(-1)^{2 m-1} \tilde{\theta}(z, \zeta) \wedge d v(z)=-\tilde{w}(z, \zeta) \wedge$ $\bar{\partial} \nu(z)$ for $\zeta \neq z$, since $\widetilde{w}(z, \zeta)$ contains the factor $d \zeta_{1} \wedge \cdots \wedge d \zeta_{m}$. Hence (7) implies

(8) $d_{z, \zeta}(f(\zeta) \wedge \tilde{w}(z, \zeta) \wedge \nu(z))=(\bar{\partial} f(\zeta)) \wedge \widetilde{w}(z, \zeta) \wedge \nu(z)-(-1)^{t} f(\zeta) \wedge \tilde{w}(z, \zeta) \wedge \bar{\partial} \nu(z)$ for $\zeta \neq z$. Then

(9) $\partial(\Omega \times \Omega \backslash U(\epsilon)) \cap\left[(\mathbf{K} \oplus \alpha \mathbf{K})^{m} \times \operatorname{supp}(\nu)\right]=(((\partial \Omega) \times \Omega) \cup(\Omega \times \partial \Omega)-\partial U(\epsilon)) \cap$ $\left[(\mathbf{K} \oplus \alpha \mathbf{K})^{m} \times \operatorname{supp}(\nu)\right]$, where $U(\epsilon):=\left\{(\zeta, z) \in(\mathbf{K} \oplus \alpha \mathbf{K})^{m} \times(\mathbf{K} \oplus \alpha \mathbf{K})^{m}: \mid \zeta-\right.$ $z \mid<\epsilon\}, 0<\epsilon<\epsilon_{0}, 0<\epsilon_{0}<\infty$, is fixed. In view of Corollary 2.3 and formulas (8), (9),

(10) ${ }_{(\partial \Omega) \times \Omega \cup \Omega \times(\partial \Omega)} P^{n}[f(\zeta) \wedge \tilde{w}(z, \zeta) \wedge \nu(z)]-\partial U(\epsilon) P^{n}[f(\zeta) \wedge \tilde{w}(z, \zeta) \wedge v(z)]=$ $\Omega^{2} \backslash U(\epsilon) P^{n}[(\bar{\partial} f(\zeta)) \wedge \widetilde{w}(z, \zeta) \wedge v(z)]-(-1)^{t} \Omega^{2} \backslash U(\epsilon) P^{n}[f(\zeta) \wedge \widetilde{w}(z, \zeta) \wedge \bar{\partial} v(z)]$.

For $B_{\epsilon}^{-}:=\left\{\zeta \in(\mathbf{K} \oplus \alpha \mathbf{K})^{m}:|\zeta|<\epsilon\right\}$, there exists $\partial B_{\epsilon}^{-}$such that $T\left(\partial B_{\epsilon}^{-} \times(\mathbf{K} \oplus \alpha \mathbf{K})^{m}\right)=$ $\partial U(\epsilon)$, where $T(\zeta, z):=(z+\zeta, z), T:(\mathbf{K} \oplus \alpha \mathbf{K})^{m} \times(\mathbf{K} \oplus \alpha \mathbf{K})^{m} \rightarrow(\mathbf{K} \oplus \alpha \mathbf{K})^{2 m}$. The differential form $v(z)$ contains the factor $d z_{1} \wedge \cdots \wedge d z_{m}$, hence $\widetilde{w}(z, \zeta) \wedge \nu(z)=w(z, \zeta) \wedge v(z)$ and $T^{*}(f(\zeta) \wedge \widetilde{w}(z, \zeta) \wedge v(z))=\sum_{|I|=t} f_{I}(z+\zeta) d(\bar{z}+\bar{\zeta})^{\wedge I} \wedge w(z, \zeta) \wedge v(z)$, where $T^{*}$ is the pullback operator on differential forms (see Section 2.2.5). The degree of $w(z, \zeta)$ is $2 m-1$ and $2 m-1=\operatorname{dim} \mathbf{K}\left(\partial B_{\epsilon}^{-}\right)$, consequently, $\left.d(\bar{z}+\bar{\zeta})^{\wedge I} \wedge w(z, \zeta)\right|_{\left(\partial B_{\epsilon}^{-}\right) \times(\mathbf{K} \oplus \alpha \mathbf{K})^{m}}=$ $\left.d \bar{z}^{\wedge I} \wedge w(z, \zeta)\right|_{\left(\partial B_{\epsilon}^{-}\right) \times(\mathbf{K} \oplus \alpha \mathbf{K})^{m}}$. 
Therefore, taking $R>0$ such that $\Omega \subset B\left((\mathbf{K} \oplus \alpha \mathbf{K})^{m}, 0, R\right)=: B_{R}: \partial U(\epsilon) P^{n}[f(\zeta) \wedge \widetilde{w}(z, \zeta)]$ $=(-1)^{t}{ }_{z \in B_{R}} P^{n}\left[\sum_{|I|=t} \zeta \in T^{-1}(\partial U(\epsilon)) P^{n}\left[f_{I}(z+\zeta) \wedge w(z, \zeta)\right] d \bar{z}^{\wedge I} \wedge v(z)\right]$, since $d \bar{z}^{\wedge I} \wedge w(z, \zeta)$ $=(-1)^{t} \tilde{w}(z, \zeta) d \bar{z}^{\wedge I}$ for $|I|=t, \Omega+\Omega \subset B_{R}+B_{R}=B_{R}$, where $\operatorname{supp}(f) \subset \Omega$ (see Lemma 2.16). In view of Theorem 3.1,

$$
\zeta \in T^{-1}(\partial U(\epsilon)) P^{n}\left[f_{I}(z+\zeta) \wedge w(z, \zeta)\right]=f_{I}(z)+\zeta \in T^{-1}(\partial U(\epsilon)) P^{n}\left\{\left[f_{I}(z+\zeta)-f_{I}(z)\right] \wedge w(z, \zeta)\right\}
$$

for $|I|=t$, which tends to $f_{I}(z)$ when $\epsilon$ tends to zero, since $\operatorname{supp}(f)$ is bounded, where $T^{-1}(\partial U(\epsilon))=\left(\partial B_{\epsilon}^{-}\right) \times(\mathbf{K} \oplus \alpha \mathbf{K})^{m}$, and inevitably

$$
\lim _{\epsilon \rightarrow 0} \partial U(\epsilon) P^{n}[f(\zeta) \wedge \tilde{\mathcal{w}}(z, \zeta) \wedge \nu(z)]=(-1)_{\Omega}^{t} P^{n}[f(z) \wedge v(z)]
$$

TheOREM 3.7. Let $M$ be a compact manifold and let $L_{\partial M}^{v, n}, R_{\partial M}^{v, n}, B_{M}^{n}$ be given by Section 3.3. Suppose $f$ is the $C^{(q, n)}-(0, t)$-differential form, $0 \leq t \leq m$. Then

(1) $(-1)^{t} f(z)=\left(L_{\partial M}^{v, n} f\right)(z)-\left(R_{\partial M}^{v, n} \bar{\partial} f+B_{M}^{n} \bar{\partial} f\right)(z)+\bar{\partial}\left(R_{\partial M}^{v, n} f+B_{M}^{n} f\right)(z)$ for each $z \in \widetilde{M}$ such that

(2) $(f \wedge \tilde{\gamma}) \circ \phi\left(z_{1}, \ldots, z_{l-1}, z_{l}+\operatorname{Exp}(\eta), z_{l+1}, \ldots, z_{m}\right)=: \tilde{\psi}_{l}(\eta) \in{ }_{s} C^{(q+1, n-1)}\left(\omega_{l, \epsilon}, L(\Lambda \mathbf{K}(\alpha)\right.$, $Y)$ ) for each $l=1, \ldots, m$ and each $\epsilon=\epsilon_{j}$, where

$$
\begin{gathered}
\omega_{l}:=\left\{\eta \in \mathbf{K}(\alpha):\left(z_{1}, \ldots, z_{l-1}, z_{l}+\operatorname{Exp}(\eta), z_{l+1}, \ldots, z_{m}\right) \in \Omega\right\}, \\
\omega_{l, \epsilon}:=\omega_{l} \backslash \log \left(B\left(\mathbf{K}(\alpha), z_{l}, \epsilon\right)\right), \quad \epsilon_{j}>0 \text { for each } j \in \mathbb{N}, \\
\lim _{j \rightarrow \infty} \epsilon_{j}=0, \quad 0 \leq q \in \mathbb{Z}, 1 \leq n \in \mathbb{N} .
\end{gathered}
$$

Proof. If $v(z, \zeta)=\bar{\zeta}-\bar{z}$, then $L_{\partial M}^{v, n}=B_{\partial M}^{n}, R_{\partial M}^{v, n}=0$, and Theorem 3.7(1) reduces to Theorem 3.6(1). If $t=0$, then by (7) and (16) in Section $3.3 B_{M}^{n} f=0$ and $R_{\partial M}^{v, n} f=0$, hence Theorem 3.7(1) reduces to Theorem 3.4(1). Assume $1 \leq t \leq m$. In view of Section 3.3 and Theorem 3.6, $L_{\partial M}^{v, n} f, R_{\partial M}^{v, n} \bar{\partial} f, B_{M}^{n} \bar{\partial} f, \bar{\partial} R_{\partial M}^{v, n} f$, and $\bar{\partial} B_{M}^{n} f$ are in $C^{(q, n)}(M, L(\Lambda \mathbf{K}(\alpha), Y))$. Using the diffeomorphism $\phi$, consider $\Omega$ instead of $M$. In view of Theorem 3.6(1) it remains to prove

(3) $\bar{\partial}\left(R_{\partial \Omega}^{v, n} f\right)(z)=\left(B_{\partial \Omega}^{n} f\right)(z)-\left(L_{\partial \Omega}^{v, n} f\right)(z)+\left(R_{\partial \Omega}^{v, n} \bar{\partial} f\right)(z)$ for each $z \in \widetilde{\Omega}$ and satisfying condition (2). Consider the differential form

(4)

$$
\begin{array}{r}
\kappa:=\sum_{j=1}^{m}(-1)^{j+1}\left(\zeta_{j}-z_{j}\right)^{-1} d \zeta_{j} \wedge_{k \neq j}\left[\left(\eta^{v}(z, \zeta, \lambda)\right)^{-1} d_{z, \zeta, \lambda} \eta_{k}^{v}(z, \zeta, \lambda)\right. \\
\left.\wedge(\xi(\zeta-z))^{-1} d_{\zeta} \xi_{k}(\zeta-z)\right] .
\end{array}
$$

In accordance with Sections 3.1 and 3.2, $\xi$ and $v$ are of class of smoothness $C^{(q, n)}$, hence $\kappa$ and $\tilde{\gamma}$ belong to $C^{(q, n)}(W, L(\Lambda \mathbf{K}(\alpha), Y))$ for suitable clopen $W \subset \Omega \times(\mathbf{K} \oplus \alpha \mathbf{K})^{m} \times$ $B(\mathbf{K}, 0,1)$ such that $\Omega \times(\partial \Omega) \times B(\mathbf{K}, 0,1) \subset W, \zeta \neq z$. Condition (7) in Section 3.2 is satisfied for $\xi(\bar{\zeta}-\bar{z})=\operatorname{Exp}\left(\pi^{-s}(\bar{\zeta}-\bar{z})\right)$ and $v(z, \zeta)$ such that $\xi(v(z, \zeta))=\operatorname{Exp}\left(\pi^{-\widetilde{\phi}(s)} v(z, \zeta)\right)$, where $\tilde{\phi}(s)$ is given by Section 3.2 and satisfies formula (3.7) and condition (2) of Theorem 3.1. Therefore, the family of such differential forms $\psi$ and $w$ is nonvoid. In view of conditions in (3) Section 3.1 and in (7) Section 3.2, in the sense of distributions, 
(5) $d_{z, \zeta, \lambda} \kappa=0$ on $W$, that is, $z \in \Omega P^{n}\left[\left(d_{z, \zeta, \lambda} \kappa\right) \wedge \nu\right]=0$ for each $\nu$ as above. From $\partial_{\zeta} \kappa=0$ and (5) it follows that $\left(\bar{\partial}_{z, \zeta}+d_{\lambda}+\partial_{z}\right)(\kappa)=0$, together with $\partial_{\zeta}(\tilde{\gamma})=0$, implies

(6) $\left(\bar{\partial}_{z, \zeta}+d_{\lambda}\right)(\tilde{\gamma})+\partial_{z}(\tilde{\gamma})+\left(\bar{\partial}_{z, \zeta}+d_{\lambda}+\partial_{z}\right)(\kappa-\tilde{\gamma})=0$ on $W, \zeta \neq z$,

since $(\kappa-\tilde{\gamma})$ contains a factor $\partial_{z} \eta_{k}^{v}$ and $\partial_{z}(\kappa-\tilde{\gamma})=0$. The monomials in $(\kappa-\tilde{\gamma})$ with respect to $d z_{j}, d \bar{z}_{j}, d \zeta_{j}, d \bar{\zeta}_{j}$, and $d \lambda$ and, consequently, in $\left(\bar{\partial}_{z, \zeta}+d_{\lambda}+\partial_{z}\right)(\tilde{\gamma}-\kappa)$ contain at least one of the differentials $d z_{1}, \ldots, d z_{m}$ as a factor. The same is true for $\partial_{z}(\tilde{\gamma})$. The monomials in $\left(\bar{\partial}_{z, \zeta}+d_{\lambda}\right)(\tilde{\gamma})$ do not contain any of the differentials $d z_{j}$. Hence from (6) it follows that $\left(\bar{\partial}_{\zeta}+d_{\lambda}\right)(\tilde{\gamma})=-\bar{\partial}_{z} \tilde{\gamma}$. Then

(7) $d_{\zeta, \lambda}(f \wedge \tilde{\gamma})=\left(\bar{\partial}_{\zeta}+d_{\lambda}\right)(f \wedge \tilde{\gamma})=(\bar{\partial} f) \wedge \tilde{\gamma}+(-1)^{t} f \wedge\left(\bar{\partial}_{\zeta}+d_{\lambda}\right) \tilde{\gamma}=(\bar{\partial} f) \wedge \tilde{\gamma}-$ $\bar{\partial}_{z}(f \wedge \tilde{\gamma})$. Applying Corollary 2.3 and formula (7) to the differential form $f \wedge \tilde{\gamma}$ on $(\partial \Omega) \times B$, where $B:=B(\mathbf{K}, 0,1)$, gives

(8) ${ }_{(\zeta, \lambda) \in(\partial \Omega) \times B} P^{n}[(\bar{\partial} f) \wedge \tilde{\gamma}]-\bar{\partial}_{z(\zeta, \lambda) \in(\partial \Omega) \times B} P^{n}[f \wedge \tilde{\gamma}]={ }_{(\partial \Omega) \times\{0\}} P^{n}[f \wedge \tilde{\gamma}]-$ $(\partial \Omega) \times\{\beta\} P^{n}[f \wedge \tilde{\gamma}]$.

On the other hand, $\left.\tilde{\gamma}\right|_{\lambda=0}=\psi,\left.\tilde{\gamma}\right|_{\lambda=\beta}=\widetilde{w}$, and formula (8) is equivalent to formula (3) due to formulas (3), (12), and (13) in Section 3.3.

Corollary 3.8. Let $M$ and $f$ be as in Theorem 3.7 and $\partial v / \partial \bar{z}=0$ on $M$. For $t=1, \ldots, m$, put

(1) $T_{t}^{n}:=(-1)^{t}\left(R_{\partial M}^{v, n}+B_{M}^{n}\right)$. Then

(2) $f(z)=\bar{\partial}\left(T_{t}^{n} f\right)(z)+\left(T_{t+1}^{n} \bar{\partial} f\right)(z)$

for each $z \in \widetilde{M}$ and satisfying Theorem 3.7(2). If $\bar{\partial} f=0$, then $u=T_{t}^{n} f$ is a solution of $\bar{\partial} u(z)=f(z)$ for each $z \in \widetilde{M}$ and $f$ satisfying Theorem 3.7(2).

Proof. In view of formula (18) in Section 3.3, $L_{\partial M}^{v, n} f={ }_{\partial M} P^{n}\left[f \wedge \Upsilon_{t}^{v}\right]$. Since $\partial v(z, \zeta) / \partial \bar{z}=$ 0 , the monomials in $\Upsilon_{t}^{v}$ of bidegree $(0, t)$ in $z$ vanish if $t \geq 1$. Therefore, $L_{\partial M}^{v, n} f=0$ and (2) follows from Theorem 3.7(1). Then from (2) it follows that $\bar{\partial} u(z)=f(z)$ if $\bar{\partial} f(z)=0$ for each $z \in \widetilde{M}$ and satisfying Theorem 3.7(2), where $u=T_{t}^{n} f$.

Definition 3.9. Let $M$ be a manifold over K satisfying 2.4 with $(q+1, n)$-antiderivationally holomorphic ${ }_{S} C^{(q+1, n-1)}$-transition maps $\phi_{i} \circ \phi_{j}^{-1}$ between charts $\left(U_{i}, \phi_{i}\right)$ and $\left(U_{j}, \phi_{j}\right)$ for each $U_{i} \cap U_{j} \neq \varnothing$ and let $G L(N, \mathbf{K}(\alpha))$ be the group of invertible $N \times N$-matrices with entries in $\mathbf{K}(\alpha)$.

(1) A $(q+1, n)$-antiderivationally holomorphic vector bundle over $\mathbf{K}(\alpha)$ of $\mathbf{K}(\alpha)$ dimension $N$ over $M$ is a ${ }_{S} C^{(q+1, n-1)}$-vector bundle over $M$ with the characteristic fiber $(\mathbf{K}(\alpha))^{N}$ and with $(q+1, n)$-antiderivationally holomorphic atlas of local trivializations of $B$, that is, with a family $\left\{U_{j}, h_{j}\right\}$ such that $\left\{U_{j}\right\}$ is a (cl)open covering of $M$, for each $j, h_{j}$ is a ${ }_{S} C^{(q+1, n-1)}$-bundle isomorphism from $\left.B\right|_{U_{j}}$ onto $U_{j} \times(\mathbf{K}(\alpha))^{N}$; the corresponding transition mappings $g_{i, j}: U_{i} \cap U_{j} \rightarrow G L(N, \mathbf{K}(\alpha))$ defined by $\left(z, g_{i, j}(z) v\right)=h_{i} \circ h_{j}^{-1}(z, v), z \in$ $U_{i} \cap U_{j}, v \in(\mathbf{K}(\alpha))^{N}$ are $(q+1, n)$-antiderivationally holomorphic ${ }_{S} C^{(q+1, n-1)}$-mappings. Equipped with the atlas $\left\{\left.B\right|_{U_{j}}, h_{j}\right\}$, the bundle $B$ gets the structure of the ${ }_{S} C^{(q+1, n-1)}-(q+$ $1, n)$-antiderivationally holomorphic manifold.

(2) $\mathrm{A}{ }_{S} C^{(q+1, n-1)}$-bundle homomorphism between ${ }_{S} C^{(q+1, n-1)}-(q+1, n)$-antiderivationally holomorphic vector bundles $B_{1}$ and $B_{2}$ is called ${ }_{S} C^{(q+1, n-1)}-(q+1, n)$-antiderivationally holomorphic if it is ${ }_{s} C^{(q+1, n-1)}-(q+1, n)$-antiderivationally holomorphic as a map between the ${ }_{S} C^{(q+1, n-1)}-(q+1, n)$-antiderivationally holomorphic manifolds 
$B_{1}$ and $B_{2}$. A ${ }_{S} C^{(q+1, n-1)}-(q+1, n)$-antiderivationally holomorphic section of a $\mathbf{K}(\alpha)_{S} C^{(q+1, n-1)}-(q+1, n)$-antiderivationally holomorphic vector bundle is defined similarly.

(3) $\mathrm{A}{ }_{S} C^{(q+1, n-1)}-(q+1, n)$-antiderivationally holomorphic vector bundle over $M$ is called ${ }_{S} C^{(q+1, n-1)}-(q+1, n)$-antiderivationally holomorphically trivial if there exists a ${ }_{S} C^{(q+1, n-1)}-(q+1, n)$-antiderivationally holomorphic bundle isomorphism from $B$ onto $M \times(\mathbf{K}(\alpha))^{N} . B$ is called ${ }_{S} C^{(q+1, n-1)}-(q+1, n)$-antiderivationally holomorphically trivial over a (cl)open set $U \subset M$ if $\left.B\right|_{U}$ is ${ }_{S} C^{(q+1, n-1)}-(q+1, n)$-antiderivationally holomorphically trivial. A ${ }_{S} C^{(q+1, n-1)}-(q+1, n)$-antiderivationally holomorphic trivialization of $B$ (over $U$ ) is a ${ }_{S} C^{(q+1, n-1)}-(q+1, n)$-antiderivationally holomorphic bundle isomorphism from $B$ onto $M \times(\mathbf{K}(\alpha))^{N}\left(\left.B\right|_{U}\right.$ onto $\left.U \times(\mathbf{K}(\alpha))^{N}\right)$.

(4) A $\mathbf{K}(\alpha)$-valued differential form of degree $r$ over $M$ can be defined as a section of the vector bundle $\Lambda^{r} T^{*}(M)_{\mathbf{K}(\alpha)}$, where $T^{*}(X)_{\mathbf{K}(\alpha)}$ is the $\mathbf{K}(\alpha)$ cotangent bundle of $M$ over scalars $b \in \mathbf{K}(\alpha)$ (see [19]). A differential form of degree $r$ with values in a ${ }_{S} C^{(q+1, n-1)}-$ $(q+1, n)$-antiderivationally holomorphic bundle (or a $B$-valued differential form) over $M$ is a section of the bundle $\Lambda^{r}\left(T^{*}(M)_{\mathbf{K}(\alpha)}\right) \otimes_{\mathbf{K}(\alpha)} B$.

If $\left\{U_{j}: j \in J\right\}$ is a (cl)open covering of $M$ such that $B$ is ${ }_{S} C^{(q+1, n-1)}-(q+1, n)$-antiderivationally holomorphically trivial over each $U_{j}$ and $\left\{g_{i, j}: i, j \in J\right\}$ is the corresponding system of transition functions, then a differential form with values in $M$ can be identified with a system $\left\{f_{j}\right\}$ of $N$-tuplets of differential forms on $U_{j}$ such that $f_{i}=g_{i, j} f_{j}$ over $U_{i} \cap U_{j}$ for each $i, j \in J$. A differential form $f$ with values in $B$ is called a $(0, t)$ form, ${ }_{s} C^{(q+1, n-1)}-(0, t)$-form, and so forth if for each (cl)open subset $U \subset M$, where $B$ is ${ }_{S} C^{(q+1, n-1)}-(q+1, n)$-antiderivationally holomorphically trivial, the corresponding $N$-tuple of differential forms on $U$ consists of $(0, t)$-forms, ${ }_{S} C^{(q+1, n-1)}-(0, t)$-forms, and so forth. Each $(s, t)$-form with values in a ${ }_{S} C^{(q+1, n-1)}-(q+1, n)$-antiderivationally holomorphic vector bundle can be identified with some $(0, t)$-forms with values in some other $n$-antiderivationally holomorphic vector bundle.

Definition 3.10. Let $M$ be a ${ }_{S} C^{(q+1, n-1)}-(q+1, n)$-antiderivationally holomorphic manifold, let $B$ be a ${ }_{S} C^{(q+1, n-1)}-(q+1, n)$-antiderivationally holomorphic vector bundle over $M$, and let $\left\{U_{j}: j \in J\right\}$ be a $(\mathrm{cl})$ open covering of $M$, where $J$ is a set. A derivationally $(q+1, n-1)$-holomorphic Cousin data in $M$ means a system $\left\{f_{i, j}: i, j \in J\right\}$ of derivationally $(q+1, n-1)$-holomorphic sections $f_{i, j}: U_{i} \cap U_{j} \rightarrow B$ such that $f_{i, j}+f_{j, k}=f_{i, k}$ in $U_{i} \cap U_{j} \cap U_{k}$ for each $i, j, k \in J$. The corresponding Cousin problem consists in finding a system $\left\{f_{j}: j \in J\right\}$ of derivationally $(q+1, n-1)$-holomorphic sections $f_{j}: U_{j} \rightarrow B$ such that $f_{i, j}=f_{i}-f_{j}$ in $U_{i} \cap U_{j}$ for each $i, j \in J$.

THEOREM 3.11. Let $M$ be $a_{S} C^{(q+1, n-1)}-(q+1, n)$-antiderivationally holomorphic manifold and let $B$ be $a{ }_{S} C^{(q+1, n-1)}-(q+1, n)$-antiderivationally holomorphic vector bundle over $M$. Consider two conditions:

(1) each derivationally $(q+2, n-1)$-holomorphic Cousin problem in B has a solution;

(2) each $B$-valued ${ }_{S} C^{(q+1, n-1)}-(0,1)$-form on $M$ such that $\bar{\partial} f=0$ on $M$ has a section $u: M \rightarrow B$ such that $\bar{\partial} u=f$ on $M$.

Then from (1), (2) follows. From (2) it follows that (1) in the class $u \in C^{(q+2, n-1)}$ and $\bar{\partial} u \in{ }_{s} C^{(q+1, n-1)}$ is $(q+1, n-1)$-antiderivationally holomorphic. 
Proof. $(1) \Rightarrow(2)$. First, that $f$ is a ${ }_{S} C^{(q+1, n-1)}$-form means that there exists a refinement $\left\{U_{k}^{\prime}: k\right\}$ of $\left\{U_{j}\right\}$ consisting of clopen $U_{k}^{\prime}$ such that $g_{k}\left(U_{k}^{\prime}\right)$ is bounded in $(\mathbf{K}(\alpha))^{N}$ and $\left.f\right|_{U_{k}^{\prime}} \in{ }_{s} C^{(q+1, n-1)}$, where $A t^{\prime}(M)=\left\{\left(U_{k}^{\prime}, g_{k}\right): k\right\}$. Choose $A t^{\prime}(M)$ such that $\bigcup_{k} \tilde{U}_{k}^{\prime}=M$. Denote $\left\{U_{k}^{\prime}: k\right\}$ by $\left\{U_{j}: j \in J\right\}$ also such that $\partial U_{k}^{\prime}$ satisfies the condition of Theorem 2.37 up to the ${ }_{S} C^{(q+1, n-1)}$-diffeomorphism. Then Theorem 2.37 on each $U_{j}$ gives a solution $u_{j}$ such that $\left(u_{i}-u_{j}\right)$ are derivationally $(q+2, n-1)$-holomorphic on $U_{i} \cap U_{j}$ and form derivationally $(q+2, n-1)$-holomorphic Cousin data in $B$. According to (1) there exists a derivationally $(q+2, n-1)$-holomorphic section $h_{j}: U_{j} \rightarrow B$ such that $u_{i}-u_{j}=h_{i}-h_{j}$ in $U_{i} \cap U_{j}$. Set $u:=u_{i}-h_{i}$ in $U_{i}$ for each $j \in J$.

$(2) \Rightarrow(1)$ in the class $u \in C^{(q+2, n-1)}$ and $\bar{\partial} u \in{ }_{S} C^{(q+1, n-1)}$ is $(q+1, n-1)$-antiderivationally holomorphic. Characteristic functions of clopen compact subsets belong to $C^{\infty}$. It is possible to take a refinement $A t^{\prime}(M)$ of $A t(M)$ such that its charts satisfy Lemma 2.16, that is, $g_{k}\left(U_{k}^{\prime}\right)$ are balls satisfying Lemma 2.16. Choose $A t^{\prime}(M)$ such that $\bigcup_{k} \tilde{U}_{k}^{\prime}=M$. Denote it also by $\operatorname{At}(M)$. Since $M$ is metrizable, it has an atlas consisting of clopen compact charts, hence $M$ has a $C^{\infty}$-partition of unity, $\chi_{k}:=\chi_{U_{k}}$. For each $i$ and $j, f_{k, j}$ is ${ }_{S} C^{(q+1, n-1)}-$ $(q+1, n-1)$-antiderivationally holomorphic, hence $\chi_{k} f_{k, j}$ is also by Lemma 2.16 ${ }_{s} C^{(q+1, n-1)}-(q+1, n-1)$-antiderivationally holomorphic for suitable refinement $\left\{U_{j}\right.$ : $j \in J\}$, since $\chi_{k} f_{k, j}=\left.f_{k, j}\right|_{\left(U_{k} \cap \operatorname{dom}\left(f_{k, j}\right)\right)}, \bar{\partial}\left(\chi_{k} f_{k, j}\right)=0$. Set $\theta_{j}:=-\sum_{k} \chi_{k} f_{k, j}$ in $U_{j}$, hence by Theorem 2.20 there exists a $C^{(q+2, n-1)}$-solution of the Cousin problem: $f_{i, j}:=\sum_{k} \chi_{k}\left(f_{i, k}+\right.$ $\left.f_{k, j}\right)=\theta_{i}-\theta_{j}$ in $U_{i} \cap U_{j} ; \bar{\partial} \theta_{i}=\bar{\partial} \theta_{j}$ in $U_{i} \cap U_{j}$. Hence by (2) there exists a section $u: M \rightarrow$ $B$ such that $\bar{\partial} u=\bar{\partial} \theta_{j}$ in $U_{j}$. The setting $h_{j}=\theta_{j}-u$ in $U_{j}$ provides (1).

Remark 3.12. Theorem 3.4(1), Theorem 3.6(1), and Theorem 3.7(1) are the non-Archimedean analogs of the Leray, Koppelman, and Koppelman-Leray formulas, respectively.

3.4. Notes and definitions. The local field $\mathbf{K}$ is the disjoint union of balls $B\left(\mathbf{K}, z_{j}, R\right)$ for a given $0<R<\infty$, where $z_{j} \in \mathbf{K}$ for each $j \in \mathbb{N}$. Therefore, the antiderivation operators ${ }_{B_{j}} P^{n}$ on $B_{j}:=B\left(\mathbf{K}, z_{j}, R\right)$ induce the antiderivation operator ${ }_{\mathbf{K}} P^{n}$ on $\mathbf{K}$ such that

$(1)_{\mathbf{K}}\left(P^{n}[f]\right)(y):=\sum_{j=1}^{\infty}\left(B_{j} P^{n}\left[f \chi_{B_{j}}\right]\right)(y)$

on $C^{(q, n-1)}(\mathbf{K}, \mathbf{L})$, where $\mathbf{K} \subset \mathbf{L} \subset \mathbf{C}_{\mathbf{p}}$, $\mathbf{L}$ is a complete field relative to its uniformity. Then

$$
\begin{aligned}
{ }_{P} C^{(q, n)}\left(\mathbf{K}^{1}, Y\right):= & { }_{\mathbf{K}} P^{n}\left(C^{(q, n-1)}\left(\mathbf{K}^{\mathbf{1}, Y)}\right) \oplus Y,\right. \\
{ }_{S} C^{(q+1, n-1)}\left(\mathbf{K}^{1}, Y\right):= & \left\{g \in C^{(q+1, n-1)}\left(\mathbf{K}^{1}, Y\right): g\left(x_{1}, \ldots, x_{l}\right) \in{ }_{P, x_{j}} C^{(q+1, n-1)}\left(\mathbf{K}^{1}, Y\right)\right. \\
& \quad \text { for each } j=1, \ldots, l\}, \\
{ }_{P, x_{j}} C^{(q+1, n-1)}\left(\mathbf{K}^{1}, Y\right):= & { }_{\mathbf{K}} P_{x_{j}}^{n}\left(C^{(q, n-1)}\left(\mathbf{K}^{l}, Y\right)\right) \oplus Y,
\end{aligned}
$$

where $C^{(q, n-1)}\left(\mathbf{K}^{1}, Y\right)$ and ${ }_{P} C^{(q, n)}\left(\mathbf{K}^{1}, Y\right)$ are supplied with the inductive limit topologies induced by the embeddings $C^{(q, n-1)}\left(B\left(\mathbf{K}^{1}, z, R^{\prime}\right), Y\right) \hookrightarrow C^{(q, n-1)}\left(\mathbf{K}^{1}, Y\right), 0<R^{\prime}<\infty$, where ${ }_{\mathbf{K}^{1}} P^{n}:={ }_{\mathbf{K}} P_{x_{1}}^{n} \cdots_{\mathrm{K}} P_{x_{l}}^{n}, x_{1}, \ldots, x_{l} \in \mathbf{K}, Y$ is a Banach space over $\mathbf{L}$ such that $\mathbf{K} \subset \mathbf{L}$ (see also $[17,20])$.

Therefore, in the standard way we get the definition of a locally compact manifold $M$ over $\mathbf{K}$ of class ${ }_{P} C^{(q, n)}$ or ${ }_{S} C^{(q+1, n-1)}$, that is, transition mappings of charts $\phi_{i, j} \in{ }_{P} C^{(q, n)}$ 
or $\phi_{i, j} \in{ }_{S} C^{(q+1, n-1)}$, where $V_{j}$ is clopen in $M, \phi_{j}\left(V_{j}\right)$ is clopen in $\mathbf{K}^{1}, 1 \leq l \in \mathbb{N}, l=$ $\operatorname{dim}_{\mathbf{K}} M$ (see Section 2.2.5). Using charts and ${ }_{P} C^{(q, n)}\left(\mathbf{K}^{\mathbf{l}}, \mathbf{K}^{\mathbf{m}}\right)$ or ${ }_{S} C^{(q+1, n-1)}\left(\mathbf{K}^{\mathbf{l}}, \mathbf{K}^{\mathbf{m}}\right)$, we get the uniform space ${ }_{P} C^{(q, n)}(M, N)$ or ${ }_{S} C^{(q+1, n-1)}(M, N)$ of all mappings $g: M \rightarrow N$ of class ${ }_{P} C^{(q, n)}$ or ${ }_{S} C^{(q+1, n-1)}$, respectively, where $M$ is the ${ }_{P} C^{(q, n)}$-manifold or ${ }_{S} C^{(q+1, n-1)}$ manifold on $\mathbf{K}^{\mathbf{l}}$ and $N$ is the ${ }_{P} C^{(q, n)}$-manifold or ${ }_{S} C^{(q+1, n-1)}$-manifold on $\mathbf{K}^{\mathbf{m}}$, respectively, that is, $\psi_{i} \circ g \circ \phi_{j}^{-1}$ is of class ${ }_{P} C^{(q, n)}$ or ${ }_{S} C^{(q+1, n-1)}$ for each $i$ and $j$ such that its domain is nonempty, where $A t(M)=\left\{\left(V_{j}, \phi_{j}\right): j\right\}, A t(N)=\left\{\left(W_{j}, \psi_{j}\right): j\right\}$. The uniformity in ${ }_{P} C^{(q, n)}\left(\mathbf{K}^{\mathbf{l}}, \mathbf{K}^{\mathbf{m}}\right)$ or ${ }_{S} C^{(q+1, n-1)}\left(\mathbf{K}^{\mathbf{l}}, \mathbf{K}^{\mathbf{m}}\right)$ induces the uniformity in ${ }_{P} C^{(q, n)}(M, N)$ or ${ }_{S} C^{(q+1, n-1)}(M, N)$, respectively (see [17, Remark 2.4]).

For a locally compact manifold $M$ over $\mathrm{K}$ of class ${ }_{P} C^{(q, n)}$ or ${ }_{S} C^{(q+1, n-1)}$, let $\operatorname{Dif} P^{(q, n)}(M)$ or Dif $S^{(q+1, n-1)}(M)$ denote a family of all diffeomorphisms $f: M \rightarrow M, f(M)=M,(f-$ id $) \in{ }_{P} C^{(q, n)}$, and $\left(f^{-1}-\mathrm{id}\right) \in{ }_{P} C^{(q, n)}$ or $(f-\mathrm{id}) \in{ }_{S} C^{(q+1, n-1)}$ and $\left(f^{-1}-\mathrm{id}\right) \in{ }_{S} C^{(q+1, n-1)}$, respectively, where $\operatorname{id}(z)=z$ for each $z \in M, M \hookrightarrow \mathbf{K}^{N},{ }_{P} C^{(q, n)}(M, M) \hookrightarrow{ }_{P} C^{(q, n)}\left(M, \mathbf{K}^{N}\right)$, ${ }_{S} C^{(q+1, n-1)}(M, M) \hookrightarrow{ }_{S} C^{(q+1, n-1)}\left(M, \mathbf{K}^{N}\right)$ such that $(f-\mathrm{id})$ is correctly defined, $N \in \mathbb{N}$.

Theorem 3.13. (1) The uniform spaces $\operatorname{Dif} P^{(q, n)}(M)$ and $\operatorname{Dif} S^{(q+1, n-1)}(M)$ are the topological groups for each $0 \leq q \in \mathbb{Z}, 1 \leq n \in \mathbb{N}$.

(2) They have embeddings as clopen subsets into ${ }_{P} C^{(q, n)}(M, M)$ and into ${ }_{S} C^{(q+1, n-1)}(M$, $M)$, respectively.

(3) The uniform spaces ${ }_{P} C^{(q, n)}(M, N), \quad{ }_{S} C^{(q+1, n-1)}(M, N), \quad \operatorname{Dif} P^{(q, n)}(M)$, and Dif $S^{(q+1, n-1)}(M)$ are complete and separable.

(4) The groups $\operatorname{Dif} P^{(q, n)}(M)$ and $\operatorname{Dif} S^{(q+1, n-1)}(M)$ are ultrametrizable when $M$ is compact.

(5) The uniform spaces $\operatorname{Dif} P^{(q, n)}(M)$ and $\operatorname{Dif} S^{(q+1, n-1)}(M)$ have the infinite-dimensional manifold structures over $\mathbf{K}$.

Proof. First, prove that compositions of diffeomorphisms preserve classes ${ }_{P} C^{(q, n)}(M, M)$ and ${ }_{S} C^{(q+1, n-1)}(M, M)$, respectively. For this consider two diffeomorphisms $\psi, \phi \in$ $\operatorname{Dif} P^{(q, n)}\left(U^{m}\right)$ or Dif $S^{(q+1, n-1)}\left(U^{m}\right)$ simultaneously. A diffeomorphism $\phi$ is called the simplest diffeomorphism if it has the coordinate form

$$
\begin{gathered}
x_{j}=\phi_{j}\left(y_{1}, \ldots, y_{m}\right)=y_{j} \quad \text { for each } j=1, \ldots, k-1, k+1, \ldots, m, \\
x_{k}=\phi_{k}\left(y_{1}, \ldots, y_{m}\right)=\phi_{k}\left(y_{1}, \ldots, y_{k}, \ldots, y_{m}\right),
\end{gathered}
$$

where $x_{j}, y_{j} \in U, x=\left(x_{1}, \ldots, x_{m}\right), m=\operatorname{dim}_{\mathbf{K}} M$. Suppose that the marked number $k$ is for $\phi$ and $l$ is for $\psi$. To prove $\phi \circ \psi \in \operatorname{Dif} P^{(q, n)}\left(U^{m}\right)$ or $\operatorname{Dif} S^{(q+1, n-1)}\left(U^{m}\right)$ it is sufficient to verify that $\left\{\phi_{k}\left(y_{1}, \ldots, y_{k-1}, \psi_{l}\left(y_{1}, \ldots, y_{m}\right), y_{k+1}, \ldots, y_{m}\right)-y_{k}\right\}$ is in ${ }_{P} C^{(q, n)}\left(U^{m}, \mathbf{K}\right)$ or ${ }_{S} C^{(q+1, n-1)}\left(U^{m}, \mathbf{K}\right)$ correspondingly.

In $C^{0}\left(U^{m}, \mathbf{K}^{m}\right)$ there exists the polynomial Amice base $\left\{\bar{Q}_{n}(x): n \in \mathbb{N}_{0}^{m}\right\}$ and it is also the base in $C^{(q, n)}\left(U^{m}, \mathbf{K}^{m}\right)$, where $\mathbb{N}_{0}:=\{j: 0 \leq j \in \mathbb{Z}\}$ (see $[1,17]$ ). The linear ordering $\triangle$ in $\mathbf{K}$ induces the linear ordering $\triangle$ in $\mathbf{K}^{m}$ and hence in $U^{m}: x \Delta y$ if and only if $x_{1}=$ $y_{1}, \ldots, x_{j-1}=y_{j-1}, x_{j} \triangle y_{j}$, where $1 \leq j \leq m, y_{j} \in \mathbf{K}, y=\left(y_{1}, \ldots, y_{m}\right)$ (see Section 2.2.1). Take, in particular, $U=B(\mathbf{K}, 0,1)$. Then $(\beta, \ldots, \beta)$ is the largest element in $U^{m}$. Let $\mathbb{Z}_{K}:=$ $\left\{z \in \mathbf{K}: z=\sum_{l=0}^{t} z_{l} \pi_{l}, 0 \leq t \in \mathbb{Z}, z_{l} \in\left\{0, \theta_{1}, \ldots, \theta_{p^{n}-1}\right\}\right\}$, then $\mathbb{Z}_{K}$ is dense in $B(\mathbf{K}, 0,1)$ and is countable. There are decompositions 
(i) $\psi_{l}(y)=\sum_{n \in \mathbb{N}_{0}{ }^{m}} a\left(n, \psi_{l}\right) \bar{Q}_{n}(y)$,

(ii) $\phi_{k}(y)=\sum_{n \in \mathbb{N}_{0} m} a\left(n, \phi_{k}\right) \bar{Q}_{n}(y)$,

where $a\left(n, \psi_{l}\right)$ and $a\left(n, \phi_{k}\right) \in \mathbf{K}$. In view of the conditions imposed on $\psi_{l}$ and $\phi_{k}$ and the continuity of the K-linear operators ${ }_{U} P_{x_{j}}^{n}$,

(iii) $\phi_{k}(y)=\left\{\sum_{n \in \mathbb{N}_{0}{ }^{m}} a\left(n, \partial \phi_{k}(y) / \partial y_{j}\right)\left(\left._{U} P_{y_{j}}^{n} \bar{Q}_{n}(y)\right|_{y_{j, 0}} ^{y_{j}}\right)\right\}+\phi_{k}\left(y_{1}, \ldots, y_{j-1}, y_{j, 0}, y_{j+1}, \ldots\right.$, $\left.y_{m}\right)$

for each $j=1, \ldots, m$ and analogously for $\psi_{l}$, where $y_{j, 0}$ and $y_{j} \in U$. To show $\left(\phi_{k}\left(y_{1}, \ldots\right.\right.$, $\left.\left.y_{k-1}, \psi_{l}(y), y_{k+1}, \ldots, y_{m}\right)-y_{k}\right) \in{ }_{s} C^{(q+1, n-1)}\left(U^{m}, \mathbf{K}\right)$, it is sufficient to find $h_{j}: U^{m} \rightarrow \mathbf{K}$ such that

(iv) $\left.{ }_{U} P_{y_{j}}^{n} h_{j}\right|_{y_{j, 0}} ^{y_{j}}=-h_{j, 0}+\phi_{k}\left(y_{1}, \ldots, y_{k-1}, \psi_{l}(y), y_{k+1}, \ldots, y_{m}\right)-y_{k}$

for each $j=1, \ldots, m$, where $h_{j, 0} \in \mathbf{K}$.

From (iii) and the continuity of the K-linear operator ${ }_{U} P_{y_{j}}^{n}$ it follows that to resolve (iv) it is sufficient to find a solution of the problem

(v) $\left.{ }_{U} P_{y_{j}}^{n} h\right|_{y_{j, 0}} ^{y_{j}}=\left(\left.{ }_{U} P_{y_{j}}^{n} y^{t^{1}}\right|_{y_{j, 0}} ^{y_{j}}\right) \cdots\left(\left.{ }_{U} P_{y_{j}}^{n} y^{t^{l}}\right|_{y_{j, 0}} ^{y_{j}}\right)$

for each $l \in \mathbb{N}$ and each $t^{k}=\left(t_{1}^{k}, \ldots, t_{m}^{k}\right) \in \mathbb{N}_{0}^{m}, k=1, \ldots, l, y^{t}=y_{1}^{t_{1}} \cdots y_{m}^{t_{m}}$. On the other hand,

(vi) ${ }_{U} P^{n} z^{t}=\sum_{0 \leq j \leq n-1, k \in \mathbb{N}_{0}} t(t-1) \cdots(t-j+1) z_{k}^{t-j}\left(z_{k+1}-z_{k}\right)^{j+1} /(j+1)$ !, where $z \in U, t \in \mathbb{N}, j \in \mathbb{Z}$. Moreover, $\left.\left(\partial / \partial y_{j}\right)_{U} P_{y_{j}}^{n}\right|_{\left(C^{(q, n-1)}\left(U^{m}, \mathbf{K}\right)\right)}=I$, hence $(\mathrm{v})$ can be simplified in the considered class of ${ }_{P, y_{j}} C_{0}^{(q+1, n-1)}\left(U^{m}, \mathbf{K}\right)$-functions acting on both sides of (v) by $\left(\partial / \partial y_{j}\right)$. For each $z \in \mathbb{Z}_{\mathbf{K}}$ there exists a solution ${ }_{z} h(y)$ of $(\mathrm{v})$ for each $y \in U^{m}$ such that $y_{j} \triangle z$, since the set $\left\{u \in \mathbb{Z}_{\mathbf{K}}: u \triangle z\right\}$ is finite. In view of (vi) and Section 2.1, this family $\left\{{ }_{z} h(y): z \in \mathbb{Z}_{\mathbf{K}}\right\}$ can be chosen consistent, that is, ${ }_{z} h(y)={ }_{\eta} h(y)$ for each $y$ such that $y_{j} \triangle \min (z, \eta)$. Therefore, there exists

(vii) $h=\lim _{z \rightarrow \beta z} h$

such that $(\mathrm{v})$ is satisfied for each $y \in U^{m}$. In particular, id $\in{ }_{S} C^{(q+1, n-1)}(M, M)$.

For the class ${ }_{P} C^{(q, n)}\left(U^{m}, \mathbf{K}\right)$ it is sufficient to find the solution of the problem

(viii) $\left(U^{m} P^{n} h\right)(y)=\left(U^{m} P^{n} y^{t^{1}}\right) \cdots\left(U^{m} P^{n} y^{t^{l}}\right)$

for each $l \in \mathbb{N}$ and each $t^{k} \in \mathbb{N}_{0}^{m}, k=1, \ldots, l,|t|:=t_{1}+\cdots+t_{m} \geq 1$. In view of (vi), Section 2.1, and ${ }_{U^{m}} P^{n}={ }_{U} P_{y_{1}}^{n} \cdots_{U} P_{y_{m}}^{n}$ there exists a consistent family ${ }_{z} h$ satisfying (viii) for each $z \in \mathbb{Z}_{\mathbf{K}}^{m}$ and each $y \triangle z$ such that ${ }_{z} h(y)={ }_{\eta} h(y)$ for each $y \triangle \min (z, \eta)$, where $\eta \in \mathbb{Z}_{\mathbf{K}}^{m}$, since the set $\left\{u \in \mathbb{Z}_{\mathbf{K}}^{m}: u \triangle z\right\}$ is finite, $\left.\left(\partial / \partial y_{1}\right) \cdots\left(\partial / \partial y_{m}\right)_{U^{m}} P^{n}\right|_{\left(C^{(q, n-1)}\left(U^{m}, \mathbf{K}\right)\right)}=I$, and the acting by $\left(\partial / \partial y_{1}\right) \cdots\left(\partial / \partial y_{m}\right)$ on both sides of (viii) simplifies it in the class of ${ }_{P} C_{0}^{(q, n)}\left(U^{m}, \mathbf{K}\right)$-functions. Then

(ix) $h=\lim _{z \rightarrow(\beta, \ldots, \beta)} z$ is the solution of (viii). Therefore, $(\phi \circ \psi(y)-y)$ and $(\phi \circ$ $\psi^{-1}(y)-y$ ) belong to ${ }_{S} C^{(q+1, n-1)}$ or ${ }_{P} C^{(q, n)}$, respectively. The proof above also shows that if a bijective surjective $\psi$ is in ${ }_{P} C^{(q, n)}(M, M)$ or in ${ }_{S} C^{(q+1, n-1)}(M, M)$, then $\psi^{-1}$ is in ${ }_{P} C^{(q, n)}(M, M)$ or in ${ }_{S} C^{(q+1, n-1)}(M, M)$, respectively, by solving the equation of the type $v(\operatorname{id}(y)+g(y))=-g(y)$ relative to the function $v$ for known $g:=\psi-$ id. Hence using charts $\left(\widetilde{V}_{j}, \tilde{\phi}_{j}\right)$ of $\widetilde{A} t(M)$ such that $\tilde{\phi}_{j}\left(\tilde{V}_{j}\right)=B \subset U^{m}+z_{j}$ with suitable $z_{j} \in \mathbf{K}^{m}$ for each $j, \tilde{A} t(M)$ is the refinement of $A t(M)$, and $B$ satisfies Lemma 2.16 (or applying the above proof to $B$ instead of $U^{m}$ ), we get 
that $\left(\tilde{\phi}_{l} \circ \phi \circ \psi^{k} \circ \tilde{\phi}^{-1}(y)-y\right)$ belongs to ${ }_{P} C^{(q, n)}$ or ${ }_{S} C^{(q+1, n-1)}$, respectively, on its domain for each $l$ and $j$, where $k=1$ or $k=-1$. Together with Lemma 2.16 it provides $\phi \circ \psi^{k} \in \operatorname{Dif} P^{(q, n)}(M)$ or $\phi \circ \psi^{k} \in \operatorname{Dif} S^{(q+1, n-1)}(M)$ correspondingly for each $k \in\{-1,1\}$.

If $M$ is compact, then ${ }_{P} C^{(q, n)}(M, Y)$ is normable for a Banach space $Y$ over $\mathbf{L}, \mathbf{K} \subset \mathbf{L}$ (see analogously [19, Lemma 2.1]). Let $V=B\left(C^{(q, n-1)}(M, Y), 0,1\right)$, consider $W:=\{f \in$ $C^{(q+1, n-1)}(M, Y): f\left(x_{1}, \ldots, x_{m}\right) \in_{P, x_{j}} C^{(q+1, n-1)}(M, Y) \cap\left(P_{x_{j}}^{n}(V) \oplus Y\right)$ for each $\left.j=1, \ldots, m\right\}$. In view of $\mathbf{K}$-convexity of $V$ the set $W$ is absolutely $\mathbf{K}$-convex (disked) and $W$ is absorbing in ${ }_{S} C^{(q+1, n-1)}(M, Y)$, since $P_{x_{j}}^{n}$ are continuous $\mathbf{K}$-linear and $V$ is absorbing in $C^{(q, n-1)}(M, Y)$. Then $W$ is bounded in the weak topology in ${ }_{S} C^{(q+1, n-1)}(M, Y)$. Therefore, the Minkowski functional on ${ }_{S} C^{(q+1, n-1)}(M, Y)$ generated by $W$ induces a norm in ${ }_{s} C^{(q+1, n-1)}(M, Y)$ (see [20, Exercise 6.204]). Each space ${ }_{P, x_{j}} C^{(q+1, n-1)}(M, Y)$ is complete (see, analogously, [19, Lemma 2.1]), since $Y$ is complete.

Consider the K-linear space $\Psi_{j}:={ }_{P, x_{j}} C^{(q+1, n-1)}(M, Y) \cap{ }_{S} C^{(q+1, n-1)}(M, Y)$ and topologies $\tau_{P, j}$ on ${ }_{P, x_{j}} C^{(q+1, n-1)}(M, Y)$ and $\tau_{S}$ on ${ }_{S} C^{(q+1, n-1)}(M, Y)$ induced by norms in these spaces, then $\left.\tau_{S}\right|_{\Psi_{j}} \subset \tau_{P, j}$ for each $j$ due to continuity of $P_{x_{j}}^{n}$ (for $M$ supplied with coordinates $x_{j}$ due to ${ }_{P} C^{(q, n)}$ - or ${ }_{S} C^{(q+1, n-1)}$-diffeomorphisms with $\Omega$ as in Section 2.2.5) and definition of $\tau_{S}$, since $\operatorname{ker}\left(P_{x_{j}}^{n}\right)=\{0\}$, and due to the open mapping [20, Theorem (14.4.1)], there exists the continuous K-linear operator

$$
\left(P_{x_{j}}^{n}\right)^{-1}:\left({ }_{P, x_{j}} C_{0}^{(q+1, n-1)}(M, Y), \tau_{P, j}\right) \longrightarrow\left(C^{(q, n-1)}(M, Y),\|*\|_{C^{(q, n-1)}(M, Y)}\right)
$$

consequently,

$$
\left(P_{x_{j}}^{n}\right)^{-1}:\left(\Psi_{j, 0}, \tau_{S} \mid \Psi_{j, 0}\right) \longrightarrow\left(C^{(q, n-1)}(M, Y),\|*\|_{C^{(q, n-1)}(M, Y)}\right)
$$

is continuous, where $\Psi_{j, 0}:=\Psi_{j} \cap{ }_{P, x_{j}} C_{0}^{(q+1, n-1)}(M, Y), \Psi_{j}=\Psi_{j, 0} \oplus Y$. Hence $C^{(q+1, n-1)}(M$, $Y)$ is complete relative to the above norm.

For noncompact $M$ using a refinement $A t^{\prime}(M)$ consisting of compact charts $\left(V_{j}^{\prime}, \phi_{j}^{\prime}\right)$ and the strict inductive limits of ${ }_{P} C^{(q, n)}\left(\bigcup_{j=1}^{l} V_{j}^{\prime}, Y\right)$ or ${ }_{S} C^{(q+1, n-1)}\left(\bigcup_{j=1}^{l} V_{j}^{\prime}, Y\right), l \in \mathbb{N}$, we get that ${ }_{P} C^{(q, n)}(M, Y)$ and ${ }_{S} C^{(q+1, n-1)}(M, Y)$ are complete relative to their uniformities (see [20, Theorems (12.1.6) and (12.1.8)]). In view of [20, Theorem (12.1.4)] these spaces are separable.

Let $(f-\mathrm{id}) \in{ }_{P} C^{(q, n)}(M, M)$ or $(f-\mathrm{id}) \in{ }_{S} C^{(q+1, n-1)}(M, M)$ such that $M$ is compact and $\max _{j, l}\left\|f_{l, j}-\mathrm{id}_{l, j}\right\|<1$, where $f_{l, j}:=\phi_{l} \circ f \circ \phi_{j}^{-1}, \operatorname{dom}\left(f_{l, j}\right)=: U_{l, j},\|*\|$ is taken of the space ${ }_{P} C^{(q, n)}\left(U_{l, j}, \mathbf{K}^{m}\right)$ or ${ }_{S} C^{(q+1, n-1)}\left(U_{l, j}, \mathbf{K}^{m}\right)$. In view of the ultrametric inequality $f_{l, j}$ is the isometry, since $\left\|f_{l, j}-\mathrm{id}_{l, j}\right\|=\sup _{n}\left|a\left(n, f_{l, j}-\mathrm{id}_{l, j}\right)\right|\left\|\bar{Q}_{n}\right\|$, where $\|*\|$ is the norm in ${ }_{P} C^{(q, n)}\left(U_{l, j}, \mathbf{K}^{m}\right)$ or in ${ }_{S} C^{(q+1, n-1)}\left(U_{l, j}, \mathbf{K}^{m}\right)$, respectively, induced by the norm in $C^{(q, n-1)}\left(U_{l, j}, \mathbf{K}^{m}\right)$ and the Minkowski functional as above. Then $\left\|g_{k, l} \circ f_{l, j}-\mathrm{id}_{k, j}\right\| \leq$ $\max \left(\left\|g_{k, l} \circ f_{l, j}-f_{l, j}\right\|,\left\|f_{l, j}-\mathrm{id}_{l, j}\right\|\right)$. Using partial difference quotients, $P^{n}$, and expansion 
coefficients in the Amice base, we get that $\max _{l, j}\left\|f_{l, j}^{-1}-\mathrm{id}_{l, j}\right\| \leq C \max _{l, j}\left\|f_{l, j}-\mathrm{id} l, j\right\|$, $C=$ const $>0$ is independent of $f$ (see the proof of [17, Theorem 2.6]), consequently, $\operatorname{Dif} P^{(q, n)}(M)$ and $\operatorname{Dif} S^{(q+1, n-1)}(M)$ are topological groups. For noncompact $M$ having $A t(M)$ with compact charts and using the strict inductive limit topology, we can take an entourage of the diagonal in ${ }_{P} C^{(q, n)}(M, M)^{2}$ or in ${ }_{S} C^{(q+1, n-1)}(M, M)^{2}$ of the form $\{f$ : $\left\|f_{l, j}-\mathrm{id}_{l, j}\right\| \leq|\pi|$ for each $\left.l, j \in \lambda\right\}$, where $\lambda$ is a finite subset in $\mathbb{N}$. In view of [18, Theorem A.4] there exists the inverse mapping $f_{l, j}^{-1}$, which is the local diffeomorphism, when $\operatorname{dom}\left(f_{l, j}\right) \neq \varnothing$. Then $\left.f\right|_{W}=\left.\mathrm{id}\right|_{W}$ for $W:=M \backslash \bigcup_{j \in \lambda} V_{j}^{\prime}$ for each $f \in \operatorname{Dif} P^{(q, n)}(M)$ and $\operatorname{Dif} S^{(q+1, n-1)}(M)$ with $W$ dependent on $f$, where $\operatorname{supp}(f):=\operatorname{cl}(\{x \in M: f(x) \neq x\})$ is compact, and a finite subset $\lambda$ of $\mathbb{N}$ is such that $\operatorname{supp}(f) \subset \bigcup_{j \in \lambda} V_{j}^{\prime}$. This implies that $f(M)=M$ and $f^{-1}(M)=M$, consequently, $\operatorname{Dif} P^{(q, n)}(M)$ and $\operatorname{Dif} S^{(q+1, n-1)}(M)$ are neighborhoods of id in ${ }_{P} C^{(q, n)}(M, M)$ and in ${ }_{S} C^{(q+1, n-1)}(M, M)$, respectively, left shifts in these groups $L_{g} f:=g^{-1} f$ imply that these groups are open in the spaces corresponding to them. Since $\operatorname{Dif} P^{(q, n)}(M)$ and $\operatorname{Dif} S^{(q+1, n-1)}(M)$ are complete, they are clopen in ${ }_{P} C^{(q, n)}(M, M)$ and ${ }_{S} C^{(q+1, n-1)}(M, M)$ respectively (see [5, Theorem 8.3.6]).

Finally, statements (4) and (5) follow from the proofs of [17, Theorems 2.4 and 3.6] modified for the classes of smoothness considered here.

3.5. Remark and definition. Let $M$ and $N$ be two locally compact $C^{(q, n)}$-manifolds over $\mathbf{K}$ and $f \in C^{1}(M, N), \operatorname{dim}_{\mathbf{K}} M=: m_{M}, \operatorname{dim}_{\mathbf{K}} N=: m_{N}$. Denote $\mathscr{E}:=\mathscr{E}(f):=\{z \in M$ : $\left.\operatorname{rang}\left(d_{z} f\right)<m_{N}\right\}$ and this set is called the set of critical values of $f$. The nonnegative Haar measure $\nu$ on $\mathbf{K}^{m_{N}}$ as the additive group induces the measure $\mu$ on $N$ with the help of charts, since $A t(N)$ has a disjoint refinement, where $v$ is normalized by the condition $\nu\left(B\left(\mathbf{K}^{m_{N}}, 0,1\right)\right)=1$.

Theorem 3.14. Let $f: M \rightarrow N$ be a $C^{l}$-mapping of a ${ }_{S} C^{(q+1, n-1)}$-manifold $M$ into a ${ }_{s} C^{(q+1, n-1)}$-manifold $N$, where $l>\max \left(m_{M}, m_{N}\right)$. Then $\mu(f(\mathscr{E}))=0$ (see Section 3.5).

Proof. Using the charts of atlases it is sufficient to prove the theorem for $f: U \rightarrow \mathbf{K}^{m_{N}}$, where $U$ is an open subset in $\mathbf{K}^{m_{N}}$. For $m_{M}=0$ and $m_{N}=0$ the statement is evident, therefore, consider $m_{M} \geq 1$ and $m_{N} \geq 1$. Put $\mathscr{E}_{i}:=\left\{y \in U: f^{(j)}(y)=0\right.$ for each $\left.j \leq i\right\}$, hence $\mathscr{E} \supset \mathscr{E}_{1} \supset \mathscr{E}_{2} \supset \ldots$. To finish the proof use the following two lemmas.

LEMMA 3.15. $\mu\left(f\left(\mathscr{E} \backslash \mathscr{E}_{1}\right)\right)=0$.

Proof. Consider $n \geq 2$, since for $n=1$ there is only one partial derivative and from $y \in \mathscr{E}$ it follows that $y \in \mathscr{E}_{1}$. Let $y \in \mathscr{E} \backslash \mathscr{E}_{1}$, then there exists a nonzero partial derivative, for example, $\partial f_{1}(x) / \partial x_{1}$ at the point $x=y$. There exists a mapping $h: U \rightarrow \mathbf{K}^{m_{N}}$ such that $h(x):=\left(f_{1}(x), x_{2}, \ldots, x_{m_{N}}\right)$ for which $\operatorname{rang}(d h(y))=m_{N}$. In view of [18, Theorem A.4] the mapping $h$ is the diffeomorphism of some open $V=V(y) \subset U$ onto a neighborhood $W \ni z:=h(y)$. The set $\mathscr{E}^{\prime}$ of critical points for $g:=f \circ h^{-1}: W \rightarrow \mathbf{K}^{m_{N}}$ coincides with $h(V \cap \mathscr{E})$, that is, $g\left(\mathscr{E}^{\prime}\right)=f(V \cap \mathscr{E})$. Consider the family $g^{t}:\left(\{t\} \times \mathbf{K}^{m_{M}-1}\right) \cap W \rightarrow\{t\} \times$ $\mathbf{K}^{m_{N}-1}$, where $t \in B(\mathbf{K}, 0,1)$. The point $b$ is critical for $g^{t}$ if and only if it is critical for $g$. In view of the induction hypothesis $\mu\left[g^{t}\left(\mathscr{E}\left(g^{t}\right)\right)\right]=0$ in $\{t\} \times \mathbf{K}^{m_{N}-1}$, hence $\mu\left(g\left(\mathscr{E}^{\prime}\right) \cap\right.$ $\left.\left(\{t\} \times \mathbf{K}^{m_{N}-1}\right)\right)=0$ for each $t \in B(\mathbf{K}, 0,1)$. From the Fubini theorem in $L^{1}\left(\mathbf{K}^{\mathbf{m}}, \mu, \mathbf{R}\right)$ it follows that $\mu\left(g\left(\mathscr{E}^{\prime}\right)\right)=0$. 
Lemma 3.16. $\mu\left(f\left(\mathscr{E}_{k}\right)\right)=0$ for each $k$ such that $1 \leq k<l$.

Proof. Take a covering of $\mathscr{E}_{k}$ by a countable number of balls of radius $\delta>0, \delta \leq \delta_{0}$, where $\delta_{0}>0$ is sufficiently small. Take one of these balls to be $B$. From the definition of $\mathscr{E}_{k}$ and the Taylor formula (see [22, Theorem 29.4] and [18, Theorem A.5]) it follows that $f(x+$ $h)=f(x)+R(x, h)$, where $\|R(x, h)\| \leq b\|h\|^{k+1}, x \in \mathscr{E}_{k}, x+h \in B, b \leq\|f\|_{C^{l}\left(U, \mathbf{K}^{m_{N}}\right)}<\infty$ for a compact clopen $U$ in $\mathbf{K}^{m_{M}}$. Divide $B$ into a disjoint union of $q^{m_{M}}$ balls of radius $\delta / q$, $q=p^{-n}$. Let $B_{1}$ be a ball of this partition such that $B_{1} \ni x$. Then each $y \in B_{1}$ has the form $y=x+h$, where $|h| \leq \delta / q$. Then $f\left(B_{1}\right) \subset B\left(\mathbf{K}^{m_{N}}, f(x), b / q^{k+1}\right)$, consequently, $f\left(\mathscr{E}_{k} \cap B\right)$ is contained in the union of $q^{m_{N}}$ balls $B_{j}$ having $\sum_{j} \mu\left(B_{j}\right) \leq q^{m_{N}}\left(b / q^{k+1}\right)^{m_{N}}=b^{m_{N}} q^{-m_{N} k}$. Then $\lim _{q \rightarrow \infty} b^{m_{N}} q^{-m_{N} k}=0$.

Therefore, Lemmas 3.15 and 3.16 finish the proof of Theorem 3.14.

Corollary 3.17. The set $N \backslash f(\mathscr{E})$ is dense in $N$, where $f \in C^{l}(M, N)$ and $l>\max \left(m_{M}, m_{N}\right)$.

Corollary 3.18. If $\operatorname{dim}_{\mathbf{K}} M<\operatorname{dim}_{\mathbf{K}} N$, then $\mu(f(M))=0$.

Definitions 3.19. A $C^{1}$-mapping $f: M \rightarrow N$ is called an immersion if $\operatorname{rang}\left(\left.d f\right|_{x}: T_{x} M \rightarrow\right.$ $\left.T_{f(x)} N\right)=m_{M}$ for each $x \in M$. An immersion $f: M \rightarrow N$ is called an embedding if $f$ is bijective.

Theorem 3.20. Let $M$ be a compact ${ }_{S} C^{(q+1, n-1)}-$ or ${ }_{P} C^{(q, n)}$-manifold over a local field $\mathbf{K}$, $\operatorname{dim}_{\mathbf{K}} M=m<\infty$. Then there exists $a_{S} C^{(q+1, n-1)}-$ or $_{P} C^{(q, n)}$-embedding $\tau: M \hookrightarrow \mathbf{K}^{2 m+1}$ and a ${ }_{S} C^{(q+1, n-1)}$ - or ${ }_{P} C^{(q, n)}$-immersion $\theta: M \rightarrow \mathbf{K}^{2 m}$, respectively. Each continuous mapping $f: M \rightarrow \mathbf{K}^{2 m+1}$ or $f: M \rightarrow \mathbf{K}^{2 m}$ can be approximated by $\tau$ or $\theta$ relative to the norm $\|*\|_{C^{0}}$.

Proof. Let $M \hookrightarrow \mathbf{K}^{N}$ be the ${ }_{S} C^{(q+1, n-1)}$ - or ${ }_{P} C^{(q, n)}$-embedding of Theorem 2.1. Consider the bundle of all $\mathbf{K}$ straight lines in $\mathbf{K}^{N}$. They compose the projective space $\mathbf{K} P^{N-1}$. Fix the standard orthonormal (in the non-Archimedean sense) base $\left\{e_{1}, \ldots, e_{N}\right\}$ in $\mathbf{K}^{N}$ and projections on $\mathbf{K}$-linear subspaces relative to this base $P^{L}(x):=\sum_{e_{j} \in L} x_{j} e_{j}$ for the $\mathbf{K}$-linear $\operatorname{span} L=\operatorname{span}_{\mathbf{K}}\left\{e_{i}: i \in \Lambda_{L}\right\}, \Lambda_{L} \subset\{1, \ldots, N\}$, where $x=\sum_{j=1}^{N} x_{j} e_{j}, x_{j} \in \mathbf{K}$ for each $j$. In this base consider the function $(x, y):=\sum_{j=1}^{N} x_{j} y_{j}$. Let $l \in \mathbf{K} P^{N-1}$, take a $\mathbf{K}$-hyperplane denoted by $\mathbf{K}_{l}^{N-1}$ and given by the condition $(x,[l])=0$ for each $x \in \mathbf{K}_{l}^{N-1}$, where $0 \neq$ $[l] \in \mathbf{K}^{N}$ characterizes $l$. Take $\|[l]\|=1$. Then the orthonormal base $\left\{q_{1}, \ldots, q_{N-1}\right\}$ in $\mathbf{K}_{l}^{N-1}$ together with $[l]=: q_{N}$ composes the orthonormal base $\left\{q_{1}, \ldots, q_{N}\right\}$ in $\mathbf{K}^{N}$ (see also [28]). This provides the projection $\pi_{l}: \mathbf{K}^{N} \rightarrow \mathbf{K}_{l}^{N-1}$ relative to the orthonormal base $\left\{q_{1}, \ldots, q_{N}\right\}$. The operator $\pi_{l}$ is $\mathbf{K}$-linear, hence $\pi_{l} \in{ }_{S} C^{(q+1, n-1)}$, since $P^{n}$ is the $\mathbf{K}$-linear operator, $\left.U P_{x_{j}}^{n} \lambda e_{j}\right|_{a} ^{b}=\lambda(b-a) e_{j}$ for each $\lambda \in \mathbf{K}$ and $a, b \in U, j=1, \ldots, N$.

To construct an immersion it is sufficient that each projection $\pi_{l}: T_{x} M \rightarrow \mathbf{K}_{l}^{N-1}$ have $\operatorname{ker}\left[d\left(\pi_{l}(x)\right)\right]=\{0\}$ for each $x \in M$. The set of all $x \in M$ for which $\operatorname{ker}\left[d\left(\pi_{l}(x)\right)\right] \neq\{0\}$ is called the set of forbidden directions of the first kind. The forbidden directions are those and only those $l \in \mathrm{K} P^{N-1}$ for which there exists $x \in M$ such that $l^{\prime} \subset T_{x} M$, where $l^{\prime}=[l]+z, z \in \mathbf{K}^{N}$. The set of all forbidden directions of the first kind forms the $C^{(q, n-1)}$ manifold $Q$ of dimension $(2 m-1)$ with points $(x, l), x \in M, l \in \mathbf{K} P^{N-1},[l] \in T_{x} M$, where $C^{(q, n)} \subset C^{(q+1, n-1)}$ for each $n \geq 1, q \geq 0$. Take $g: Q \rightarrow \mathbf{K} P^{N-1}$ given by $g(x, l):=l$. Then $g$ is of class $C^{(q, n-1)}$. In view of Theorem $3.14 \mu(g(Q))=0$ if $N-1>2 m-1$, that is, $2 m<N$. 
In particular, $g(Q)$ is not contained in $\mathrm{K}^{N-1}$ and there exists $l_{0} \notin g(Q)$, consequently, there exists $\pi_{l_{0}}: M \rightarrow \mathbf{K}_{l_{0}}^{N-1}$. Since ${ }_{S} C^{(q+1, n-1)}$ or ${ }_{P} C^{(q, n)}$ respectively is dense in $C^{(q, n-1)}$, then there exists a mapping $\kappa$ such that $\kappa \in{ }_{S} C^{(q+1, n-1)}$ or $\kappa \in{ }_{P} C^{(q, n)}$ is sufficiently close to $\pi_{l_{0}}$ relative to $\|*\|_{C^{1}}$ correspondingly such that $\kappa \circ \theta$ is the immersion, since $M$ is compact. In view of Theorem 3.13 the composition $\kappa \circ \theta$ is of class ${ }_{S} C^{(q+1, n-1)}$ or ${ }_{P} C^{(q, n)}$ correspondingly. This procedure can be prolonged, when $2 m<N-k$, where $k$ is the number of the steps of projection. Hence $M$ can be immersed in $\mathbf{K}^{2 m}$.

Consider now the forbidden directions of the second type $l \in \mathbf{K} P^{N-1}$, for which there exists $x \neq y \in M$ simultaneously belonging to $l$ after suitable parallel translation $[l] \mapsto$ $[l]+z, z \in \mathbf{K}^{N}$. The set of the forbidden directions of the second type forms the manifold $S:=M^{2} \backslash \Delta$, where $\Delta:=\{(x, x): x \in M\}$. Consider $\psi: S \rightarrow \mathbf{K} P^{N-1}$, where $\psi(x, y)$ is the straight K-line with the direction vector $[x, y]$ in the orthonormal base. Then $\mu(\psi(S))=$ 0 in $\mathrm{K} P^{N-1}$ if $2 m+1<N$. Then the closure $\mathrm{cl}(\psi(P))$ coincides with $\psi(P) \cup g(Q)$ in $\mathrm{K} P^{N-1}$. Hence there exists $l_{0} \notin \mathrm{cl}(\psi(P))$. Then consider $\pi_{l_{0}}: M \rightarrow \mathbf{K}_{l_{0}}^{N-1}$. Since ${ }_{S} C^{(q+1, n-1)}$ or ${ }_{P} C^{(q, n)}$ correspondingly is dense in $C^{(q, n-1)}$, then there exists a mapping $\kappa$ such that $\kappa \in{ }_{S} C^{(q+1, n-1)}$ or $\kappa \in{ }_{P} C^{(q, n)}$ is sufficiently close to $\pi_{l_{0}}$ relative to $\|*\|_{C^{1}}$ such that $\kappa \circ \tau$ is the embedding, since $M$ is compact. In view of Theorem 3.13 the composition $\kappa \circ \tau$ is of class ${ }_{S} C^{(q+1, n-1)}$ or ${ }_{P} C^{(q, n)}$ correspondingly. This procedure can be prolonged, when $2 m+1<N-k$, where $k$ is the number of the steps of projection. Hence $M$ can be embedded into $\mathbf{K}^{2 m+1}$.

Remark 3.21. Theorems 3.14 and 3.20 are non-Archimedean analogs of the Sard and Whitney theorems. In Theorem 3.20 classes of smoothness globally on $M$ are important. Theorem 3.20 justifies the considered class of manifolds $M$ in the theorems above about antiderivational representations of functions.

3.6. Note and definition. The proof of Theorem 3.13 shows that the family of all diffeomorphisms of $M$ of the class ${ }_{P} C((t, s))$ as defined slightly differently in [19] also forms the topological group. Moreover, spaces ${ }_{P} C((t, s), \Omega \rightarrow Y):=P(l, s)[C((t, s-1), \Omega \rightarrow Y)] \oplus Y$ and ${ }_{P} C^{(t, s)}(M, Y)$ are topologically $\mathbf{K}$-linearly isomorphic, where $l=[t]+1,[t]$ is the integer part of $t,[t] \leq t, 0 \leq t \in \mathbb{R}$, although the antiderivation operators $P(l, s)$ on a clopen subset $X^{\prime}=\Omega$ in $B\left(\mathbf{K}^{m}, 0,1\right)$ (see $\left[15\right.$, Section 2.11] and [16]) and ${ }_{\Omega} P^{s}$ above (see Sections 2.1 and 2.2.5) are different.

Define by induction spaces ${ }_{S}^{l} C^{\xi+(l, 0)}(\Omega, Y):=\left\{f \in C^{\xi+(l, 0)}(\Omega, Y): f\left(x_{1}, \ldots, x_{m}\right) \in\right.$ ${ }_{U} P_{x_{j}}^{n+l}\left({ }_{S}^{l-1} C^{\xi+(l-1,0)}(\Omega, Y)\right) \oplus Y$ for each $\left.j=1, \ldots, m\right\}$, where $l \in \mathbb{N},{ }_{S}^{1} C^{\xi+(1,0)}(\Omega, Y):=$ ${ }_{S} C^{\xi+(1,0)}(\Omega, Y),{ }_{S}^{0} C^{\xi}(\Omega, Y):=C^{\xi}(\Omega, Y)$.

Theorem 3.22. Let $M$ be a ${ }_{S}^{l} C^{(q+l, n-1)}$-manifold over $\mathbf{K}$ with $l \geq 2$, then there exists a clopen neighborhood $\widetilde{T} M$ of $M$ in $T M$ and an exponential ${ }_{S}^{l} C^{(q+l, n-1)}$-mapping $\exp : \widetilde{T} M \rightarrow M$ of $\widetilde{T} M$ on $M$.

Proof. As in the proof of [19, Theorem 2.4] it can be shown that the non-Archimedean geodesic equation $\nabla_{\dot{c}} \dot{c}=0$ with initial conditions $c(0)=x_{0}, \dot{c}(0)=y_{0}, x_{0} \in M, y_{0} \in T_{x_{0}} M$ has a unique ${ }_{S}^{l} C^{(q+l, n-1)}$-solution, $c: B(\mathbf{K}, 0,1) \rightarrow M$. For a chart $\left(U_{j}, \phi_{j}\right)$ containing $x$, put 
$\psi_{j}(b)=\phi_{j} \circ c(b)$, then

$$
\begin{aligned}
& \psi_{j}(b)=\phi_{j}\left(x_{0}\right)+{ }_{U} P^{q+l+n}\left(y_{0}+{ }_{U} P^{q+l+n-1} f\right), \\
& \psi_{j}(b)=\psi_{j}\left(b ; x_{0}, y_{0}\right), \quad b \in B(\mathbf{K}, 0,1),
\end{aligned}
$$

where $f \in{ }_{S}^{l-2} C^{(q+l+n-3)}\left(B(\mathbf{K}, 0,1), \mathbf{K}^{m}\right)$, consequently, the mapping $V_{1} \times B\left(\mathbf{K}^{m}, 0, \delta\right) \ni$ $\left(\tilde{x}_{0}, y_{0}\right) \mapsto \psi_{j}\left(\beta ; x_{0}, y_{0}\right)$ is of class of smoothness ${ }_{S}^{l} C^{(q+l, n-1)}$, where $0<\delta, \tilde{x}_{0}=\phi_{j}\left(x_{0}\right) \in$ $V_{1} \subset V_{2} \subset \phi_{j}\left(U_{j}\right), V_{1}$ and $V_{2}$ are clopen, $\delta$ and $V_{1}$ are sufficiently small, to satisfy the inclusion $\psi_{j}\left(\beta ; x_{0}, y_{0}\right) \in V_{2}$ for each $\left(\tilde{x}_{0}, y_{0}\right) \in V_{1} \times B\left(\mathbf{K}^{m}, 0, \delta\right)$. See [19, Section 2.4] the rest of the proof.

Theorem 3.23. Let $\Omega=\Omega_{1} \times \cdots \times \Omega_{m}$ be a polydisk in $(\mathbf{K} \oplus \alpha \mathbf{K})^{m}$ and let

$$
{ }_{S} \bar{C}^{(q+1, n-1)}(\Omega, \mathbf{K}(\alpha)):=\left\{f \in{ }_{S} C^{(q+1, n-1)}(\Omega, \mathbf{K}(\alpha)): \bar{\partial} f=0 \text { on } \Omega\right\}
$$

then $\left.{ }_{S} \bar{C}^{(q+1, n-1)}(\Omega, \mathbf{K}(\alpha))\right|_{\widetilde{\Omega}}$ is the algebra over $\mathbf{K}$, where $\widetilde{\Omega}:=\left\{z \in \Omega: z_{j}\right.$ is encompassed by $\left.\partial \Omega_{j}\right\}$ for each $j=1, \ldots, m, z=\left(z_{1}, \ldots, z_{m}\right)$.

Proof. Evidently ${ }_{s} \bar{C}^{(q+1, n-1)}(\Omega, \mathbf{K}(\alpha))$ is the $\mathbf{K}$-linear space, since $\bar{\partial}(\lambda f)=\lambda \bar{\partial} f$ for each $\lambda \in \mathbf{K}$ and $\bar{\partial}(f+g)=\bar{\partial} f+\bar{\partial} g$ for each $f, g \in{ }_{S} C^{(q+1, n-1)}(\Omega, \mathbf{K}(\alpha))$. It remains to verify that $\left.\left.f g\right|_{\widetilde{\Omega}} \in{ }_{S} \bar{C}^{(q+1, n-1)}(\Omega, \mathbf{K}(\alpha))\right|_{\widetilde{\Omega}}$ for each $f$ and $g \in{ }_{S} \bar{C}^{(q+1, n-1)}(\Omega, \mathbf{K}(\alpha))$, where as in Lemma $\left.2.16{ }_{s} \bar{C}^{(q+1, n-1)}(\Omega, \mathbf{K}(\alpha))\right|_{\widetilde{\Omega}}=\left\{\left.h\right|_{\widetilde{\Omega}}: h \in{ }_{S} \bar{C}^{(q+1, n-1)}(\Omega, \mathbf{K}(\alpha))\right\}$. In view of Theorem 2.20(i), if $f$ and $g \in{ }_{S} \bar{C}^{(q+1, n-1)}(\Omega, \mathbf{K}(\alpha))$, then $f$ and $g$ are locally $z$-analytic on $\widetilde{\Omega}$, consequently, $f g$ is locally $z$-analytic on $\widetilde{\Omega}$. In view of $(2.34)$ or by direct computation,

(i) $\operatorname{res} \xi(z-\xi)^{j}=0$ for each $-1 \neq j \in \mathbb{Z}$ and each $\xi \in \widetilde{\Omega}$,

since $\operatorname{res} \xi h=0$ for each $h$ having a decomposition of the form (2.33) with $a_{-1}=a_{-1}(h)$ $=0$; indeed it is true for the particular $h(\beta)=h(0)$ for a loop $\gamma$ encompassing 0 and such that $h(x):=\operatorname{Exp}[j \log (\gamma(x))]$ and $j \in \mathbb{Z}, x \in B(\mathbf{K}, 0,1)$, which leads to the general case.

On the other hand, $(f g)^{\prime}(z)$ also is locally $z$-analytic on $\widetilde{\Omega}$. Therefore, $\gamma_{j} P^{n}\left[(f g)^{\prime}(z) d z_{j}\right]$ $=0$ and particularly

(ii) ${ }_{\gamma_{j}} P^{n}\left[\left(\partial(f(z) g(z)) / \partial z_{j}\right) d z_{j}\right]=0$

for each loop $\gamma_{j}$ in $\Omega_{j}$ encompassed by $\partial \Omega_{j}$ (see Theorem 2.13), where $z=\left(z_{1}, \ldots, z_{m}\right.$ ), $\Omega=\Omega_{1} \times \cdots \times \Omega_{m}, \Omega_{j}$ is a ball in $\mathbf{K} \oplus \alpha \mathbf{K}, z_{j} \in \mathbf{K} \oplus \alpha \mathbf{K}$ for each $j=1, \ldots, m$. Then

$$
\begin{gathered}
\frac{\partial\left({ }_{\gamma_{j}} P^{n}\left[\left(\partial(f(z) g(z)) / \partial z_{j}\right) d z_{j}\right]\right)}{\partial z_{j}}=\frac{\partial(f g)(z)}{\partial z_{j}}, \\
{ }_{\gamma_{j}} P^{n}\left[\left(\frac{\partial(f g)(z)}{\partial z_{j}}\right) d z_{j}\right]= \\
(f g)\left(z_{1}, \ldots, z_{j-1}, \gamma_{j}(\beta), z_{j+1}, \ldots, z_{m}\right) \\
-(f g)\left(z_{1}, \ldots, z_{j-1}, \gamma_{j}(0), z_{j+1}, \ldots, z_{m}\right) .
\end{gathered}
$$


Moreover,

$$
\begin{aligned}
\gamma_{j} P^{n} & {\left[h_{j}(z) d z_{j}\right] } \\
& ={ }_{B} P^{n}\left[h_{j}\left(z_{1}, \ldots, z_{j-1}, \gamma_{j}(\zeta), z_{j+1}, \ldots, z_{m}\right) d \gamma_{j}(\zeta)\right], \\
\gamma_{j} P^{n}[ & {\left.\left[\frac{\partial(f g)(z)}{\partial z_{j}}\right) d z_{j}\right] } \\
& ={ }_{B} P^{n}\left[v_{j}\left(z_{1}, \ldots, z_{j-1}, \gamma_{j}(\zeta), z_{j+1}, \ldots, z_{m}\right) d \gamma_{j}(\zeta)\right], \\
\partial \frac{{ }_{B} P^{n}}{\partial \zeta} & {\left[h_{j}\left(z_{1}, \ldots, z_{j-1}, \gamma_{j}(\zeta), z_{j+1}, \ldots, z_{m}\right) d \gamma_{j}(\zeta)\right] } \\
& =h_{j}\left(z_{1}, \ldots, z_{j-1}, \gamma_{j}(\zeta), z_{j+1}, \ldots, z_{m}\right) \gamma^{\prime}(\zeta), \\
\partial \frac{{ }_{B} P^{n}}{\partial \zeta} & {\left[v_{j}\left(z_{1}, \ldots, z_{j-1}, \gamma_{j}(\zeta), z_{j+1}, \ldots, z_{m}\right) d \gamma_{j}(\zeta)\right] } \\
& =v_{j}\left(z_{1}, \ldots, z_{j-1}, \gamma_{j}(\zeta), z_{j+1}, \ldots, z_{m}\right) \gamma^{\prime}(\zeta), \quad \gamma_{j} \in{ }_{P} C^{n}(B, \mathbf{K}(\alpha)),
\end{aligned}
$$

where $v_{j}(z):=\partial(f g)(z) / \partial z_{j}, \zeta \in B:=B(\mathbf{K}, 0,1)$. Proceeding as in the proof of Theorem 3.13 with the help of (i), (ii), and formula (1) in Section 2.2.5 (see (iii)-(vii) in Theorem $3.13)$, find $h_{j} \in C^{(q, n-1)}(\Omega, \mathbf{K}(\alpha))$ such that $h_{j}$ is locally $z$-analytic and ${ }_{\gamma_{j}} P^{n}\left[h_{j}\left(z_{1}, \ldots\right.\right.$, $\left.\left.z_{j-1}, \zeta_{j}, z_{j+1}, \ldots, z_{m}\right) d \zeta_{j}\right]=(f g)(z)-(f g)\left(z_{0}\right)$ for each $z \in \widetilde{\Omega}$ and each $j=1, \ldots, m$, where $\gamma_{j}$ is a path with $\gamma_{j}(0)=z_{j, 0} \in \widetilde{\Omega}_{j}, \gamma_{j}(\beta)=z_{j}$ for each $j=1, \ldots, m$. This means that $\left.(f g) \in{ }_{S} \bar{C}^{(q+1, n-1)}(\Omega, \mathbf{K}(\alpha))\right|_{\widetilde{\Omega}}$, since

$$
\begin{aligned}
\frac{\partial_{\gamma_{j}} P^{n}}{\partial x_{j}} & {\left[h_{j}\left(z_{1}, \ldots, z_{j-1}, \zeta_{j}, z_{j+1}, \ldots, z_{m}\right) d \zeta_{j}\right] } \\
& =\frac{\partial_{\gamma_{j}} P^{n}}{\partial z_{j}=h_{j}(z)}\left[h_{j}\left(z_{1}, \ldots, z_{j-1}, \zeta_{j}, z_{j+1}, \ldots, z_{m}\right) d \zeta_{j}\right], \\
\frac{\partial_{\gamma_{j}} P^{n}}{\partial y_{j}} & {\left[h_{j}\left(z_{1}, \ldots, z_{j-1}, \zeta_{j}, z_{j+1}, \ldots, z_{m}\right) d \zeta_{j}\right]=\alpha h_{j}(z) }
\end{aligned}
$$

(see formulas (i) and (ii) in Section 2.2.6) such that $\left.{ }_{U} P_{x_{j}}^{n} h_{j}\right|_{x_{j, 0}} ^{x_{j}}$ and $\left.{ }_{U} P_{y_{j}}^{n} h_{j}\right|_{y_{j, 0}} ^{y_{j}}$ as particular cases of $\gamma_{j}$ along axes $x_{j}$ and $y_{j}$ give the desired result.

Corollary 3.24. The space $\left.{ }_{S} \bar{C}^{(q+1, n-1)}(\Omega, \mathbf{K}(\alpha))\right|_{\tilde{\Omega}}$ contains all locally $z$-analytic functions on $\widetilde{\Omega}$.

Proof. Mention that $1 \in C^{(q, n-1)}(\Omega, \mathbf{K}(\alpha))$ and $\left.{ }_{U} P_{x}^{n} 1\right|_{x_{0}} ^{x}=x-x_{0},\left.{ }_{U} P_{y}^{n} 1\right|_{y_{0}} ^{y}=y-y_{0}, \gamma_{j} P^{n} 1=$ $\gamma_{j}(\beta)-\gamma_{j}(0)=z_{j}-z_{j, 0}$, where $\gamma_{j} \subset \widetilde{\Omega}_{j}$, hence $z_{j}-z_{j, 0} \in{ }_{S} \bar{C}^{(q+1, n-1)}(\Omega, \mathbf{K}(\alpha))$ for each $z_{j}$ and $z_{j, 0} \in \widetilde{\Omega}_{j}=\pi_{j}\left(\widetilde{\Omega}_{j}\right)$. It is possible to take $\gamma_{j}$ contained in balls $B$ such that $B \subset \Omega_{j}$. Therefore, ${ }_{\gamma} P^{n} \sum_{l=1}^{k} \chi_{B_{l}}=\sum_{l=1}^{k} a_{l \gamma_{j}} P^{n} \chi_{B_{l}} \in{ }_{s} \bar{C}^{(q+1, n-1)}\left(\Omega_{j}, \mathbf{K}(\alpha)\right)$, where $B_{l}$ are balls satisfying conditions of Lemma 2.16, $a_{l} \in \mathbf{K}(\alpha), k \in \mathbb{N}$. In view of Theorem 3.23 each polynomial in $z$ belongs to $\left.{ }_{S} \bar{C}^{(q+1, n-1)}(\Omega, \mathbf{K}(\alpha))\right|_{\tilde{\Omega}}$. Using expansions into series by $z$ of locally $z$-analytic functions and limits of sequences of polynomials in $z$ and Lemma 2.16 leads to the conclusion that each locally $z$-analytic function on $\widetilde{\Omega}$ belongs to $\left.{ }_{S} \bar{C}^{(q+1, n-1)}(\Omega, \mathbf{K}(\alpha))\right|_{\Omega}$. 
Note 3.25. From Corollary 3.24 it follows that a ${ }_{s} \bar{C}^{(q+1, n-1)}$-manifold $M$ is locally $z$ analytic manifold and there exists a refinement $\tilde{A} t(M)=\left\{\left(\tilde{U}_{j}, \tilde{\phi}_{j}\right): j\right\}$ of $\operatorname{At}(M)$ such that transition mappings $\tilde{\phi}_{l} \circ \tilde{\phi}_{j}^{-1}$ are $z$-analytic for each $\tilde{U}_{j} \cap \widetilde{U}_{l} \neq \varnothing$. If $f$ is $z$-analytic on $\widetilde{\Omega}$, then $f^{\prime}$ is $z$-analytic on $\widetilde{\Omega}$. Therefore, there exists a family $\Upsilon$ of the cardinality $\operatorname{card}(\Upsilon)=\mathrm{c}:=\operatorname{card}(\mathbf{R})$ of all functions $f \in{ }_{S} \bar{C}^{(q+1, n-1)}(\Omega, \mathbf{K}(\alpha))$ and $f$ is not $z$-analytic on $\widetilde{\Omega}$, since a locally $z$-analytic function is not necessarily $z$-analytic. For example, take $h \in C^{(q, n-1)}(\Omega, \mathbf{K}(\alpha))$ locally $z$-analytic on $\widetilde{\Omega}$ and nonanalytic on $\widetilde{\Omega}$ and put $f(z)={ }_{\gamma} P^{n} h$, where $\gamma(0)=z_{0}, \gamma(\beta)=z, \Omega$ is a polydisk (see Corollary 3.24). Indeed, each locally polynomial in $z$ nonpolynomial $h: \Omega \rightarrow \mathbf{K}(\alpha)$ and its iterated antiderivatives along paths $h_{k}(z):={ }_{\gamma} P^{k} h_{k-1}, k=1, \ldots, n, h_{0}:=h$, up to order $n$, fit this construction. For nonlocally compact fields there is the theory of analytic elements [6].

Corollary 3.26. Let $\mathbf{L}$ be a non-Archimedean field such that $\mathbf{K}(\alpha) \subset \mathbf{L}$ with a valuation $|\cdot|_{\mathbf{L}}$ extending that of $\mathbf{K}(\alpha)$ and let $\mathbf{L}$ be complete relative to $|\cdot|_{\mathbf{L}}$. Suppose $\Omega$ is a clopen compact subset in $(\mathbf{K} \oplus \alpha \mathbf{K})^{m}$ and $Y$ is a Banach space over $\mathbf{L}$. Then $\left.f \in{ }_{S} \bar{C}^{(q+1, n-1)}(\Omega, Y)\right|_{\Omega}$ if and only if there exists an open subset $W$ in $\mathbf{L}^{m}$ and a locally $z$-analytic function $F$ on $W$, $z \in W$, such that $W \cap(\mathbf{K} \oplus \alpha \mathbf{K})^{m} \supset \widetilde{\Omega}$ and $\left.F\right|_{\widetilde{\Omega}}=f$.

Proof. The valuation group $\Gamma_{\mathbf{K}(\alpha)}$ is discrete, hence $Y$ as the $\mathbf{K}(\alpha)$-linear space has an orthonormal base $\left\{e_{j}: j \in \Lambda\right\}$, where $\Lambda$ is a set (see [28, Chapter 5]). Therefore, $F: W \rightarrow Y$ has the decomposition $F(z)=\sum_{j \in \Lambda} F_{j}(z) e_{j}$, where $F_{j}: W \rightarrow \mathbf{K}(\alpha)$. Since $F$ is locally $z$ analytic, then $f$ is locally $z$-analytic on $\widetilde{\Omega}$ and in accordance with Corollary $3.24 f \in$ $\left.{ }_{S} \bar{C}^{(q+1, n-1)}(\Omega, Y)\right|_{\widetilde{\Omega}}$. Conversely, if $\left.f \in{ }_{S} \bar{C}^{(q+1, n-1)}(\Omega, Y)\right|_{\tilde{\Omega}}$, then by Theorem $2.20 f$ is locally $z$-analytic on $\widetilde{\Omega}$, consequently, for each $\zeta \in \widetilde{\Omega}$ there exists a ball $B(\mathbf{K}(\alpha), \zeta, R(\zeta))$ with $0<R(\zeta)<\infty$ on which the power series 2.20(2) is uniformly convergent, that is, $\lim _{|k| \rightarrow 0}\left|a_{k}\right|_{\mathbf{L}} R^{|k|}=0$, hence this series is uniformly convergent on $B(\mathbf{L}, \zeta, R(\zeta))$ also. Put $W=\bigcup_{\zeta \in \widetilde{\Omega}} B(\mathbf{L}, \zeta, R(\zeta))$.

3.7. Definition and note. Let $\mathbf{L}, \Omega=\Omega(f), W=W(f)$ be satisfying conditions of Corollary 3.26 with $m=1$. Let also $T \in L(Y)$ be a bounded L-linear operator on a Banach space $Y$ over $\mathbf{L}$ with a nonvoid spectrum

$$
\sigma(T):=\{b \in \mathbf{L}:(b I-T) \text { is not invertible in } L(Y)\}
$$

(see [28, Chapter 6]), where $L(X, Y)$ is the Banach space of all bounded L-linear operators $T: X \rightarrow Y$ for Banach spaces $X$ and $Y$ over $\mathbf{L},\|T\|:=\sup _{0 \neq x \in X}\|T x\| /\|x\|, L(Y):=$ $L(Y, Y)$. Suppose, in addition, that for each $z \in W$ with $\operatorname{dist}(z, \Omega)<\infty$ there exist $R \geq$ $\operatorname{dist}(z, \Omega)$ and $\zeta \in \Omega$ such that $B(\mathbf{L}, z, R) \subset W$ and $B(\mathbf{L}, z, R) \cap \Omega=B(\mathbf{K} \oplus \alpha \mathbf{K}, \zeta, R) \subset \Omega$. Denote by $\mathscr{F}(T)$ a family of all functions $f$ with $\psi_{f} \in{ }_{S} \bar{C}^{(q+1, n-1)}\left(\omega_{\epsilon}, \mathbf{L}\right.$ ) (see Theorem 3.23), where $W$ is a clopen neighborhood of $\sigma(T), W=W(f), \Omega=W \cap(\mathbf{K} \oplus \alpha \mathbf{K}) \neq \varnothing$, $0<\operatorname{dist}(\partial \Omega, \sigma(T)):=\inf _{z \in \partial \Omega} \operatorname{dist}(z, \sigma(T)), \operatorname{dist}(z, G):=\inf _{y \in G}|z-y|$ for $G \subset \mathbf{L}$ and $z \in$ $\mathbf{L}, 0 \leq q \in \mathbb{Z}, 1 \leq n \in \mathbb{N}, \psi_{f}(\eta):=f(z+\operatorname{Exp}(\eta)), \omega:=\omega(z):=\{\eta \in \mathbf{K}(\alpha): z+\operatorname{Exp}(\eta) \in$ $\Omega\}, z \in \Omega, \omega_{\epsilon}=\omega \backslash \log (B(\mathbf{K}(\alpha), z, \epsilon)), \epsilon=\epsilon_{j}, \epsilon_{j}>0$ for each $j \in \mathbb{N}, \lim _{j \rightarrow \infty} \epsilon_{j}=0$, there exists a locally $z$-analytic function $\Psi_{f}$ on $W$ such that $\left.\Psi_{f}\right|_{\widetilde{\Omega}}=\psi_{f}$ (see Corollary 3.26). Put

$$
\text { (1) } f(T)=C(\alpha)^{-1} \partial \Omega P^{n}[f(\zeta) R(\zeta ; T) d \zeta]
$$


where $R(\zeta ; T)=(\zeta I-T)^{-1}$ for $\zeta \in \rho(T):=\mathbf{L} \backslash \sigma(T)$ and the antiderivative is supposed to be convergent in the strong operator topology sense, that is, $\partial_{\Omega} P^{n}[f(\zeta) R(\zeta ; T) y d \zeta]$ converges for each $y \in Y$. There are other definitions of spectral sets (see [28, Chapter 6]), but this one is used here.

Theorem 3.27. Let $\sigma(T) \neq \varnothing, \sigma(T) \subset \mathbf{L}, f, g \in \mathscr{F}(T), a, b \in \mathbf{L}$ (see Section 3.7). Then

(i) $a f+b g \in \mathscr{F}(T)$ and $a F(T)+b g(T)=(a f+b g)(T)$;

(ii) $f g \in \mathscr{F}_{(}(T)$ and $f(T) g(T)=(f g)(T)$;

(iii) if $f(z)=\sum_{k=0}^{\infty} a_{k} z_{k}$ on $W(f)$ such that $W(f) \supset \sigma(T)$, then $f(T)=\sum_{k=0}^{\infty} a_{k} T^{k}$.

Proof. Section 3.7 is correct, since $x I-T$ is invertible in $L(Y)$ for each $x \in \rho(T):=\mathbf{L} \backslash$ $\sigma(T)$, hence $r_{\sigma}(T):=\sup _{x \in \sigma(T)}|x| \leq\|T\|$, where $\rho(T)$ is open in $\mathbf{L}$ and $R(x ; T)$ is locally $x$-analytic on $\rho(T)$ (see [3, Chapter VII], [4], and [28, Chapter 6]).

(i) follows from Section 3.7 and Corollary 3.26.

(ii) In view of Corollary 3.26 and Theorem $3.23 f g \in \mathscr{F}(T)$, since $W(f) \cap W(g)=$ : $W(f g) \supset \sigma(T)$. Without loss of generality take $\Omega(f)$ encompassed by $\partial \Omega(g)$ shrinking $\Omega(f)$ a little if necessary such that $W(f) \supset \sigma(T), W(f) \subset W(g)$. Then

$$
\begin{aligned}
& f(T) g(T)=C(\alpha)_{\zeta \in \partial \Omega(f)}^{-2} P^{n}[f(\zeta) R(\zeta ; T) d \zeta]_{\kappa \in \partial \Omega(g)} P^{n}[g(\kappa) R(\kappa ; T) d \kappa] \\
&=C(\alpha)^{-2}{ }_{\kappa \in \partial \Omega(g)} P^{n}[\zeta \in \partial \Omega(f) \\
&\left.P^{n}[f(\zeta) g(\kappa)\{R(\zeta ; T) R(\kappa ; T)\} d \zeta] d \kappa\right] .
\end{aligned}
$$

On the other hand, $R(\zeta ; T) R(\kappa ; T)=(R(\zeta ; T)-R(\kappa ; T))(\kappa-\zeta)^{-1}$. Therefore,

$$
\begin{aligned}
& f(T) g(T)= C(\alpha)^{-2}{ }_{\zeta \in \partial \Omega(f)} P^{n}\left[f(\zeta) R(\zeta ; T)\left\{{ }_{\kappa \in \partial \Omega(g)} P^{n}\left[g(\kappa)(\kappa-\zeta)^{-1} d \kappa\right]\right\} d \zeta\right] \\
&-C(\alpha)^{-2}{ }_{\kappa \in \partial \Omega(g)} P^{n}[g(\kappa) R(\kappa ; T)\{\zeta \in \partial \Omega(f) \\
&\left.\left.P^{n}\left[f(\zeta)(\kappa-\zeta)^{-1} d \zeta\right]\right\} d \kappa\right] .
\end{aligned}
$$

The second term on the right-hand side of (3.39) is zero, since $\partial \Omega(f)$ is encompassed by $\partial \Omega(g), \kappa \in \partial \Omega(g), \zeta \in \partial \Omega(f)$ (see formulas 2.20(2), (3), (4)). Hence

$$
f(T) g(T)=C(\alpha)^{-1}{ }_{\zeta \in \partial \Omega(f)} P^{n}[f(\zeta) g(\zeta) R(\zeta ; T) d \zeta]=(f g)(T)
$$

(iii) follows from Section 3.7 and formulas 2.20(2), (3), (4) applied to $f(\zeta) R(\zeta ; T) y$ for each $y \in Y$.

Theorem 3.28. Let $\sigma(T) \neq \varnothing, \sigma(T) \subset \mathbf{L}, f \in \mathscr{F}(T)$ (see Section 3.7). Then $f(\sigma(T))=$ $\sigma(f(T))$.

Theorem 3.29. Let $\sigma(T) \neq \varnothing, \sigma(T) \subset \mathbf{L}, f \in \mathscr{F}(T), g \in \mathscr{F}(f(T))$ (see Section 3.7), and $h(z):=g(f(z))$ for each $z \in f^{-1}[W(g) \cap f(W(f))]$. Then $h \in \mathscr{F}(T)$ and $h(T)=g(f(T))$.

Proof. Theorem 3.28 follows from Theorem 3.27 analogously to [3, Theorems VII.3.10], [4], and $[9,3.3 .6]$. The function $f$ is locally $z$-analytic on $W(f), g$ is locally $z$-analytic on $W(g)$, hence $h$ is locally $z$-analytic on $f^{-1}[W(g) \cap f(W(f))]$. In view of Theorem 3.28 $\sigma(f(T)) \subset f(W(f)) \cap W(g)$, hence $h$ is defined on open $W(h)$ such that $W(h) \supset \sigma(T)$. 
Without loss of generality take $W(g) \supset f(W(f))$. Put

$$
S(\kappa)=C(\alpha)^{-1} \underset{\zeta \in \partial \Omega(f)}{ } P^{n}\left[R(\zeta ; T)(\kappa-f(\zeta))^{-1} d \zeta\right]
$$

then in accordance with Theorems 3.27 and 2.8 (applied onto pieces of $\Omega(f)$ affine homotopic to points) $(\kappa I-T) S(\kappa)=S(\kappa)(\kappa I-T)=I$, consequently, $S(\kappa)=R(\kappa ; T)$. Therefore,

$$
\begin{aligned}
g(f(T)) & =C(\alpha)^{-1} \partial \Omega(g) P^{n}[g(\kappa) R(\kappa ; f(T)) d \kappa] \\
& =-C(\alpha)^{-2} \partial \Omega(g) P^{n}\left[\partial \Omega(f) P^{n}\left\{g(\kappa) R(\zeta ; T)(\kappa-f(\zeta))^{-1} d \zeta\right\} d \kappa\right] \\
& =C(\alpha)^{-1} \partial \Omega(f) P^{n}[R(\zeta ; T) g(f(\zeta)) d \zeta]=h(T) .
\end{aligned}
$$

Proposition 3.30. Let $f_{k} \in \mathscr{F}(T)$ for each $k \in \mathbb{N}$ (see Section 3.7) and there exists a clopen subset $W$ in $\mathrm{L}$ such that $\sigma(T) \subset W \subset \bigcap_{n=1}^{\infty} W\left(f_{n}\right)$. If $f_{k}$ converges to $f$ uniformly on $W$, then $f_{n}(T)$ converges to $f(T)$ uniformly on each totally bounded subset in $Y$.

Proof. There exists a sequence $C(\alpha)^{-1} \partial_{\Omega} P^{n}\left[f_{k}(\zeta) R(\zeta ; T) d \zeta\right]$ in $L(X, Y)$ in the topology of pointwise convergence, where $L(X, Y)$ denotes the Banach space of continuous $L$-linear operators $S: X \rightarrow Y$ for two Banach spaces $X$ and $Y$ over $L$. In view of [20, Theorem (11.6.3) and Example 11.202.(g)], this sequence converges to an L-linear operator on $Y$ uniformly on each totally bounded subset in $Y$.

Definition 3.31. A point $z_{0} \in \sigma(T)$ is called an isolated point of a spectrum $\sigma(T)$, if there exists a neighborhood $U$ of $z_{0}$ such that $\sigma(T) \cap U=\left\{z_{0}\right\}$, where $U$ satisfies the same conditions of Section 3.7 as $W$ does. An isolated point $z_{0} \in \sigma(T)$ is called a pole of an operator $T$ or a pole of a spectrum, if a mapping $R(\zeta ; T)$ has a pole at $z_{0}$. An order $j\left(z_{0}\right)$ of a pole $z_{0}$ is an order of $z_{0}$ as a pole of $R(\zeta ; T)$.

Theorem 3.32. Let $f, g \in \mathscr{F}(T)$ (see Section 3.7). Then $f(T)=g(T)$ if and only if $f(\zeta)=$ $g(\zeta)$ on a clopen $W$ such that $\sigma(T) \backslash \bigcup_{l \in \Lambda}\left\{z_{l}\right\} \subset W \subset \mathbf{L}$, where $z_{l} \in \Omega \subset \mathbf{K} \oplus \alpha \mathbf{K}$ is a pole for each $l \in \Lambda, \Lambda$ is a finite set, and $(f-g)$ at $z_{l}$ has zero of order not less than $j\left(z_{l}\right)$ for each $l=1, \ldots, k$.

Proof. Without loss of generality take $g=0$ and let $f=0$ on $W \backslash \bigcup_{l \in \Lambda}\left\{z_{l}\right\}$. Then due to Theorem 2.8 (applied onto each piece affine homotopic to a point)

$$
f(T)=C(\alpha)^{-1} \sum_{l \in \Lambda} \partial_{l} P^{n}[f(\zeta) R(\zeta ; T) d \zeta]
$$

where $B_{l}:=B\left(\mathbf{K} \oplus \alpha \mathbf{K}, z_{l}, R_{l}\right), 0<R_{l}<\infty$, and $B_{l} \cap \sigma(T)=\left\{z_{l}\right\}$ for each $l \in \Lambda$. Since $f(\zeta) R(\zeta ; T)$ is regular on $B_{l}$, then by Theorem $2.32 f(T)=0$. Conversely, let $f(T)=0$, then by Theorem $3.28 f(\sigma(T))=0$. The set $\sigma(T) \cap(\mathbf{K} \oplus \alpha \mathbf{K})$ is compact and it can be covered by a finite union of balls $B\left(\mathbf{K} \oplus \alpha \mathbf{K}, \zeta_{j}, R_{j}\right), 0<R_{j}<\infty$. If $B\left(\mathbf{K} \oplus \alpha \mathbf{K}, \zeta_{j}, R_{j}\right) \cap \sigma(T)$ is infinite, then for each limit point $x$ of the latter set there exists a clopen neighborhood $V_{x}$ on which $\left.f\right|_{V_{x}}=0$ (see Theorem 2.25). Therefore, $\sigma(T) \cap\left((\mathbf{K} \oplus \alpha \mathbf{K}) \backslash \bigcup_{x} V_{x}\right)$ consists of a finite number of isolated points $\left\{\lambda_{l}: l=1, \ldots, k\right\}$, since $\Omega \supset(\mathbf{K} \oplus \alpha \mathbf{K}) \cap \sigma(T), \Omega$ is compact. Let $f \neq 0$ on any neighborhood of $\lambda_{1}$. Since $\lambda_{1} \in \sigma(T)$ and $f(\sigma(T))=\{0\}$, 
then $f$ has a zero of finite order $j$, hence $g_{1}(z)=\left(\lambda_{1}-z\right)^{j} / f(z)$ is locally $z$-analytic on a neighborhood of $\lambda_{1}$. From the proof of Theorem 3.23 it follows that

(1) $R(\zeta ; T)=\sum_{m=-\infty}^{\infty} a_{m}\left(\lambda_{1}-\zeta\right)^{m}$

on $B(\epsilon):=B\left(\mathbf{K} \oplus \alpha \mathbf{K}, \lambda_{1}, \epsilon\right)$ for a sufficiently small $0<\epsilon<\infty$, where

(2) $a_{-m}=-C(\alpha)^{-1} \partial B(\epsilon) P^{n}\left[\left(\lambda_{1}-\zeta\right)^{m-1} R(\zeta ; T) d \zeta\right]=-\left(\lambda_{1} I-T\right)^{m-1} h(T)$,

$h(T)$ denotes a function equal to 1 on $B(\epsilon)$ and zero on a neighborhood of $\lambda_{l}$ for each $l \neq 1$ such that $\psi(\eta)=h(z+\operatorname{Exp}(\eta))$ satisfies Section 3.7, which is possible due to Lemma 2.16 and Corollary 3.26, since $\operatorname{Exp}(\eta)$ is locally $\eta$-analytic. Then $a_{-m-1}=-\left(\lambda_{1} I-T\right)^{m} h(T)=$ 0 for each $m \geq j$.

3.8. Definition and note. A subset $V$ of $\sigma(T)$ clopen in $\sigma(T)$ is called a spectral set if it has a clopen neighborhood $W_{V}$ satisfying the same conditions of Section 3.7 as $W$ and $W_{V} \cap(\sigma(T) \backslash V)=\varnothing$. In accordance with Lemma 2.16 and Theorem 3.23 consider $f \in \mathscr{F}(T)$ such that $\left.f\right|_{V}=1$ and $\left.f\right|_{\sigma(T) \backslash V}=0$, which is possible due to Corollary 3.26, since $\operatorname{Exp}(\eta)$ is locally $\eta$-analytic. Put $E(V ; T):=f(T)$. In view of Theorem $3.32 E(V ; T)$ depends on $V$, but not on a concrete choice of $f$ from its definition. If $V \cap \sigma(T)=\varnothing$, put $E(V ; T)=0$. Write also $E(z ; T):=E(\{z\} ; T)$ for a singleton $\{z\}$. An index $j=j(z)$ of $z \in \mathbf{L}$ is the smallest integer $j$ such that $(z I-T)^{j} y=0$ for each $y \in Y$ with $(z I-T)^{j+1} y=0$.

Theorem 3.33. Let $T, W, \Omega, \mathbf{K}(\alpha)$ be as in Corollary 3.26, Section 3.7. If $z_{0}$ is a pole of $T$ of order $j$, then $z_{0} \in \Omega$ has the index $j$. An isolated point $z_{0} \in \sigma(T)$ is a pole of order $j$ if and only if

(i) $\left(z_{0} I-T\right)^{j} E\left(z_{0} ; T\right)=0,\left(z_{0} I-T\right)^{j-1} E\left(z_{0} ; T\right) \neq 0$.

Proof. In view of formulas (1), (2) in the proof of Theorem $3.32 z_{0}$ is a pole of order $j$ if and only if (i) is satisfied, since $a_{-m-1}=-\left(z_{0} I-T\right)^{m} E\left(z_{0} ; T\right)$. The rest of the proof is analogous to that of [3, Theorem VII.3.18] and [4] due to Corollary 3.26 and Theorem 3.23.

In view of Theorem 3.27(ii)

$$
E(V ; T) E(V ; T)=E(V ; T) \text { for each spectral set } V,
$$

that is, $E(V ; T)$ is the projection operator on $Y$ (see [28, Chapter 3$])$.

Theorem 3.34. Let $f \in \mathscr{F}(T)$ (see Section 3.7) and let $V$ be a spectral set of $f(T)$. Then $\sigma(T) \cap f^{-1}(V)$ is the spectral set of $T$ and $E(V ; f(T))=E\left(f^{-1}(V) ; T\right)$.

Proof. Let $h_{V} \in \mathscr{F}(T)$ such that $h_{V}(z)=1$ on a neighborhood $V_{1}$ of $V, h_{V}(z)=0$ on a neighborhood $V_{2}$ of $\sigma(f(T)) \backslash V_{1}$, where $V_{1} \cap V_{2}=\varnothing$, which is possible due to Theorem 3.23, Corollary 3.26, and Lemma 2.16, since $\operatorname{Exp}(\eta)$ is locally $\eta$-analytic. Then $h_{V}(f(T))$ $=E(V ; f(T))$. In view of Theorem $3.28 \sigma(T)=f^{-1}(V) \cup f^{-1}(\sigma(f(T)) \backslash V)$, where $f^{-1}(V) \cap f^{-1}(\sigma(f(T)) \backslash V)=\varnothing$. Since $f$ is continuous, then $f^{-1}(V)$ and $f^{-1}(\sigma(T) \backslash V)$ are clopen in $\sigma(T)$. Therefore, $\sigma(T) \cap f^{-1}(V)=: \Upsilon$ is the spectral set of T. Put $t_{\Upsilon}(z):=$ $h_{V}(f(z))$, then $E(\Upsilon ; T)=t_{\Upsilon}(T)$, since $t_{\Upsilon} \in \mathscr{F}(T)$ due to Corollary 3.26. From Theorem 3.29 it follows that $E(V ; f(T))=E(\Upsilon ; T)=E\left(f^{-1}(V) ; T\right)$. 
Remark 3.35. In the non-Archimedean case the Gelfand-Naimark theorem [4, Theorem IX.3.7] and [3] is not true (see [28, Chapter 6]). Therefore, the existence of the projection operator $E(V ; T)$ for each spectral set $V$ does not imply a spectral projection-valued measure decomposition of $T$ (see also [12]). Here we consider a particular class of operators satisfying conditions of Section 3.7 for which the operator $E(V ; T)$ is defined for each spectral set $V, V \subset \sigma(T)$. Put $Y_{V}:=E(V ; T) Y$. In view of Theorem 3.27(ii) and Section 3.8 $T Y_{V} \subset Y_{V}$, where $Y_{V}$ is the $\mathbf{L}$-linear subspace in $Y$, since $E(V ; T)$ is L-linear, denote $T_{V}:=\left.T\right|_{Y_{V}}$.

Theorem 3.36. Let $V$ be a spectral set of $\sigma(T) \neq \varnothing$ (see Section 3.7). Then $\sigma\left(T_{V}\right)=V$. If $f \in \mathscr{F}(T)$, then $f \in \mathscr{F}_{F}\left(T_{V}\right)$ and $f\left(T_{V}\right)=f(T)_{V}$. A point $z_{0} \in V \cap \Omega$ is the pole of $T$ of order $j$ if and only if $z_{0} \in \Omega$ is the pole of $T_{V}$ of order $j$.

Proof. Take a marked point $z \in V$ and suppose $z \notin \sigma\left(T_{V}\right)$. In view of Corollary 3.26 there exists a function $g \in \mathscr{F}(T)$ such that $\left.g\right|_{V_{1}}=0$ on a neighborhood $V_{1}$ of $V$ and $g(\zeta)=$ $\left(z_{0}-\zeta\right)^{-1}$ for each $\zeta \in V_{2}$, where $V_{2}$ is open in $\mathrm{L}, V_{1} \cap V_{2}=\varnothing, V_{2} \supset \sigma(T) \backslash V$. In view of Theorem 3.27(ii) $g(T)(z I-T)=(z I-T) g(T)=I-E\left(V\right.$; T). Then $V \subset \sigma\left(T_{V}\right)$ as in [3, Theorem VII.3.20] and [4].

Conversely, let $z \notin V$. Consider $h \in \mathscr{F}(T)$ (see Section 3.7) such that $\left.h(\zeta)\right|_{V_{1}}=(z-$ $\zeta)^{-1}$ and $\left.h\right|_{V_{2}}=0$, where $V_{1}$ is chosen such that $z \notin V_{1}, V_{1}$ is a neighborhood of $V, V_{2}$ is as above. Then by Theorem 3.27(ii) $h(T)(z I-T)=(z I-T) h(T)=E(V ; T)$. Therefore, $h(T)_{V}\left(z I_{V}-T_{V}\right)=\left(z I_{V}-T_{V}\right) h\left(T_{V}\right)=I_{V}$, since $z \notin \sigma\left(T_{V}\right)$, consequently, $\sigma\left(T_{V}\right) \subset V$ and $R\left(z ; T_{V}\right)=R(z ; T)_{V}$. Take $f \in \mathscr{F}(T)$ and a neighborhood $W$ of $\sigma(T)$ as in Section 3.7. Then

$$
\begin{aligned}
f(T)_{V} & =C(\alpha)^{-1}{ }_{\partial \Omega} P^{n}[f(z) R(z ; T) d z]_{V} \\
& =C(\alpha)^{-1}{ }_{\partial \Omega} P^{n}\left[f(z) R\left(z ; T_{V}\right) d z\right]=f\left(T_{V}\right), \\
E(z ; T) E(V ; T) & =E(z ; T) \quad \text { for each } z \in V,
\end{aligned}
$$

hence $(z I-T)^{k} E(z ; T)=\left(z I_{V}-T_{V}\right)^{k} E(z ; T)$ for each $k \in \mathbb{N}$. In view of Theorem 3.33 $z_{0} \in \Omega \cap V$ is a pole of $T$ of order $j$ if and only if it is a pole of $T_{V}$ of order $j$.

Corollary 3.37. The mapping $E \mapsto E(V ; T)$ is the isomorphism of the algebra $\Upsilon$ of all clopen spectral subsets $V$ of $\sigma(T)$ satisfying conditions of Section 3.7 on the Boolean algebra $\{E(V ; T): V \in \Upsilon\}$.

Proof. In view of Theorem 3.27 the mapping $V \mapsto E(V ; T)$ is the homomorphism. If $E(V ; T)=0$, then $Y_{V}=0$ and $\sigma\left(T_{V}\right)=\varnothing$, hence $V=\sigma\left(T_{V}\right)=\varnothing$ by Theorem 3.36. If $V_{1}, V_{2} \in \Upsilon$, then evidently $W_{V_{1}} \cup W_{V_{2}}$ and $W_{V_{1}} \cap W_{V_{2}}$ (for $V_{1} \cap V_{2} \neq \varnothing$ ) satisfy conditions of Section 3.7 as $W$. Consider $\sigma(T) \backslash V$ for $V \in \Upsilon$, then $W_{V} \cap(\sigma(T) \backslash V)=\varnothing$ (see Corollary 3.24), hence $W \backslash W_{V}$ satisfies conditions of Section 3.7 as $W$, since either each two balls in $\mathbf{L}$ are disjoint or one of them is contained in another. Therefore, $\Upsilon$ is the Boolean algebra and hence $\{E(V ; T): V \in \Upsilon\}$ is the Boolean algebra.

Note 3.38. In Sections 3.7-3.37 the generalization can be taken instead of $\Omega$ for a manifold $M$ which is ${ }_{S} C^{(q+1, n-1)}$-diffeomorphic with $\Omega$. 


\section{References}

[1] Y. Amice, Interpolation p-adique, Bull. Soc. Math. France 92 (1964), 117-180.

[2] M. Berz, Cauchy theory on Levi-Civita fields, Proceedings of the 7th International Conference on $p$-Adic Functional Analysis. Ultrametric Functional Analysis (Nijmegen, 2002) (W. H. Schikhof, C. Perez-Garcia, and A. Escassut, eds.), Contemp. Math., vol. 319, American Mathematical Society, Rhode Island, 2003, pp. 39-52.

[3] N. Dunford and J. T. Schwartz, Linear Operators. I. General Theory, Pure and Applied Mathematics, vol. 7, Interscience, New York, 1958.

[4] _ Linear Operators. Part II: Spectral Theory. Self Adjoint Operators in Hilbert Space, Interscience, New York, 1963.

[5] R. Engelking, General Topology, Mir, Moscow, 1986.

[6] A. Escassut, Analytic Elements in p-Adic Analysis, World Scientific Publishing, New Jersey, 1995.

[7] J. Fresnel and M. van der Put, Géométrie analytique rigide et applications [Rigid Analytic Geometry and Its Applications], Progress in Mathematics, vol. 18, Birkhäuser Boston, Massachusetts, 1981.

[8] G. Henkin and J. Leiterer, Theory of Functions on Complex Manifolds, Monographs in Mathematics, vol. 79, Birkhäuser Verlag, Basel, 1984.

[9] R. V. Kadison and J. R. Ringrose, Fundamentals of the Theory of Operator Algebras. Vol. I, Pure and Applied Mathematics, vol. 100, Academic Press, New York, 1983.

[10] N. Koblitz, p-Adic Numbers, p-Adic Analysis, and Zeta-Functions, Springer-Verlag, New York, 1977.

[11] - p-Adic Analysis: A Short Course on Recent Work, London Mathematical Society Lecture Note Series, vol. 46, Cambridge University Press, Cambridge, 1980.

[12] S. Ludkovsky and B. Diarra, Spectral integration and spectral theory for non-Archimedean Banach spaces, Int. J. Math. Math. Sci. 31 (2002), no. 7, 421-442.

[13] S. V. Ludkovsky, Embeddings of non-Archimedean Banach manifolds into non-Archimedean Banach spaces, Uspekhi Mat. Nauk 53 (1998), no. 5, 241-242.

[14]_. Measures on groups of diffeomorphisms of non-Archimedean manifolds, representations of groups and their applications, Theoret. and Math. Phys. 119 (1999), no. 3, 698-711.

[15] Q Quasi-invariant measures on non-Archimedean groups and semigroups of loops and paths, their representations. I, Ann. Math. Blaise Pascal 7 (2000), no. 2, 19-53.

[16] Quasi-invariant measures on non-Archimedean groups and semigroups of loops and paths, their representations. II, Ann. Math. Blaise Pascal 7 (2000), no. 2, 55-80.

[17] - A structure and representations of diffeomorphism groups of non-Archimedean manifolds, Southeast Asian Bull. Math. 26 (2003), no. 6, 975-1004.

[18] Q Quasi-invariant and pseudo-differentiable measures with values in non-Archimedean fields on a non-Archimedean Banach space, J. Math. Sci. (N. Y.) 122 (2004), no. 1, 29492983.

[19] Stochastic processes and antiderivational equations on non-Archimedean manifolds, Int. J. Math. Math. Sci. 2004 (2004), no. 29-32, 1633-1651.

[20] L. Narici and E. Beckenstein, Topological Vector Spaces, Monographs and Textbooks in Pure and Applied Mathematics, vol. 95, Marcel Dekker, New York, 1985.

[21] W. H. Schikhof, Non-Archimedean Calculus, Report, vol. 7812, Katholieke Universiteit Mathematisch Instituut, Nijmegen, 1978.

[22] Ultrametric Calculus, Cambridge Studies in Advanced Mathematics, vol. 4, Cambridge University Press, Cambridge, 1984.

[23] B. V. Shabat, Introduction to Complex Analysis. Part I. Functions of a Single Variable, 3rd ed., Nauka, Moscow, 1985. 
[24] Introduction to Complex Analysis. Part II. Functions of Several Variables, 3rd ed., Nauka, Moscow, 1985.

[25] È. Spenér, Algebraic Topology, Izdat. Mir, Moscow, 1971, translated from English by B. M. Pranov and edited by A. M. Vinogradov.

[26] M. van der Put, Algèbres de fonctions continues p-adiques. I, Nederl. Akad. Wetensch. Indag. Math. 30 (1968), 401-411.

[27] Algèbres de fonctions continues p-adiques. II, Nederl. Akad. Wetensch. Indag. Math. 30 (1968), 412-420.

[28] A. C. M. van Rooij, Non-Archimedean Functional Analysis, Monographs and Textbooks in Pure and Applied Math., vol. 51, Marcel Dekker, New York, 1978.

[29] A. Weil, L'Intègration Dans Les Groupes Topologiques Et Ses Applications, Actual. Sci. Ind., no. 869, Hermann et Cie., Paris, 1940.

[30] _ Basic Number Theory, Classics in Mathematics, Springer-Verlag, Berlin, 1995.

S. V. Ludkovsky: Theoretical Department, Institute of General Physics, Russian Academy of Sciences, 38 Vavilov Street, Moscow 119991, GSP-1, Russia

E-mail address: ludkovsk@fpl.gpi.ru 


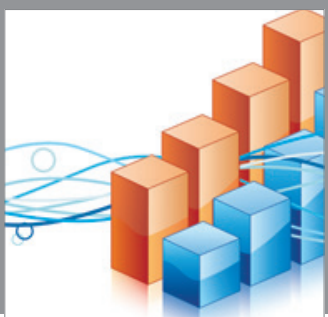

Advances in

Operations Research

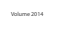

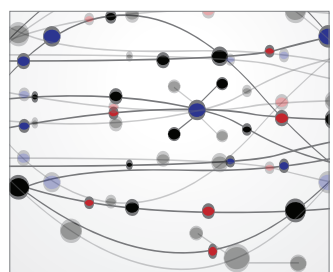

\section{The Scientific} World Journal
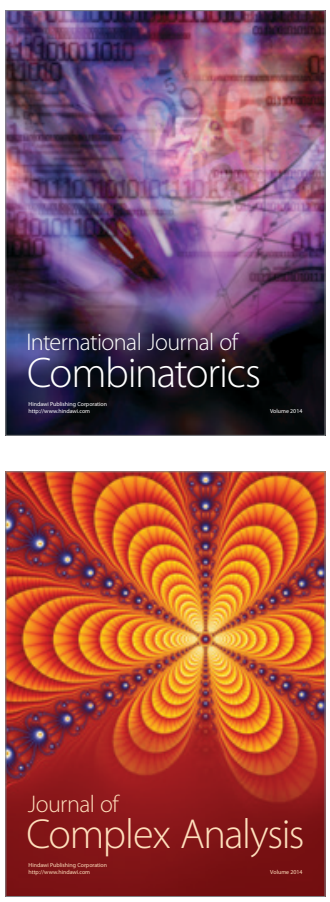

International Journal of

Mathematics and

Mathematical

Sciences
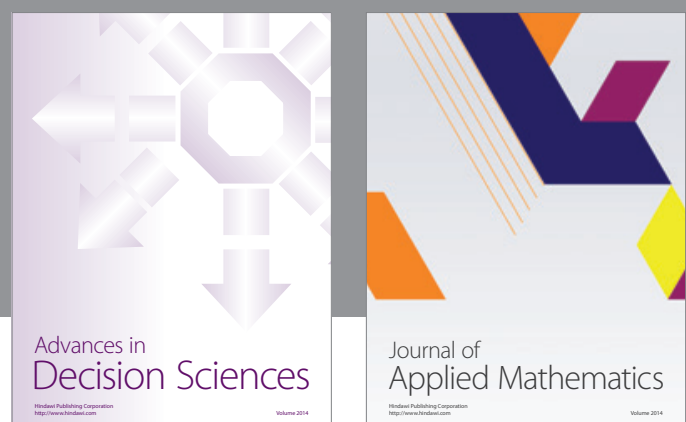

Journal of

Applied Mathematics
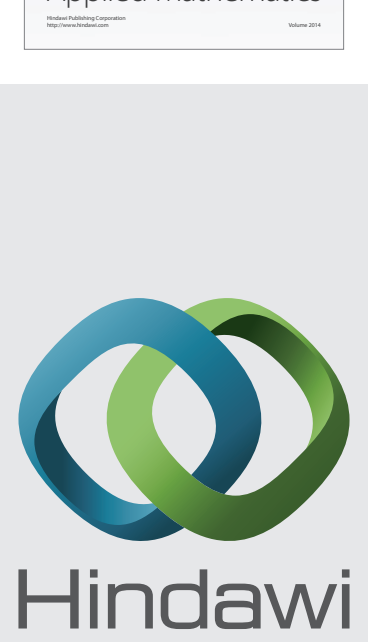

Submit your manuscripts at http://www.hindawi.com
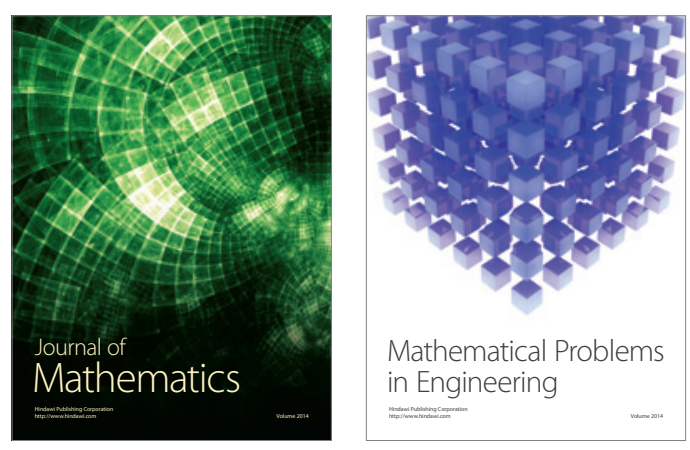

Mathematical Problems in Engineering
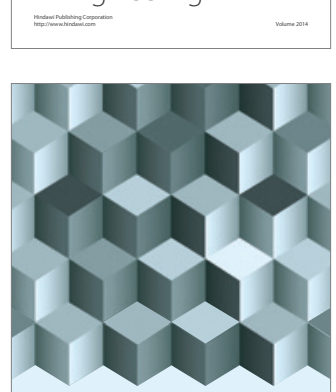

Journal of

Function Spaces
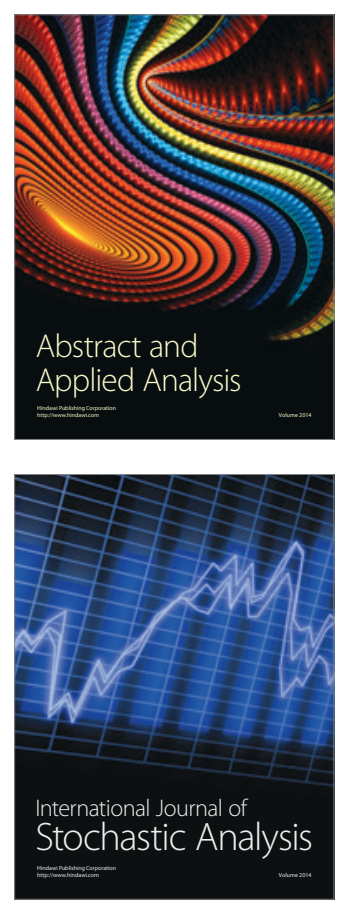

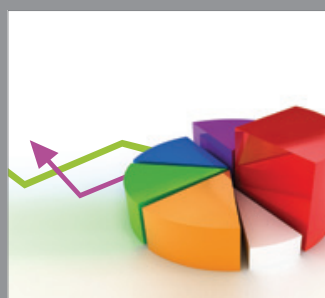

ournal of

Probability and Statistics

Promensencen
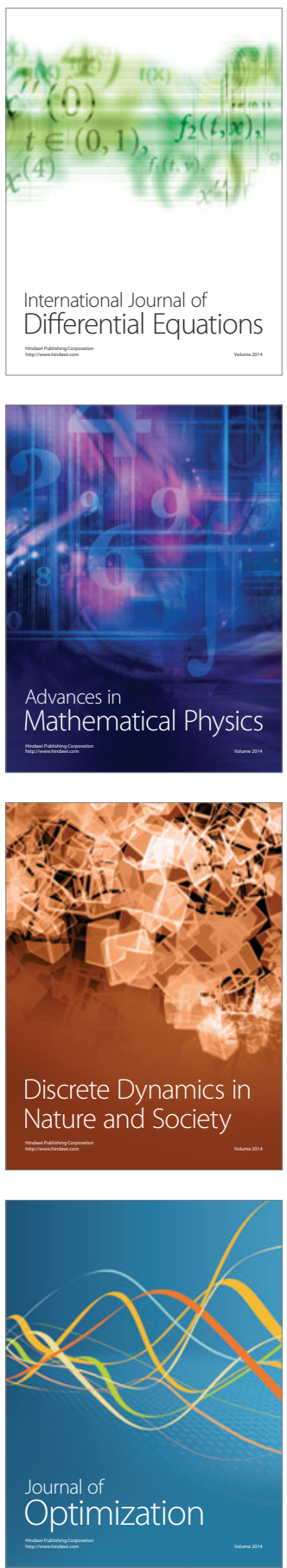